\title{
HUBBLE SPACE TELESCOPE PLANETARY CAMERA IMAGES OF R136 ${ }^{1}$
}

\author{
Bel CAMPBell
}

Department of Physics and Astronomy, University of New Mexico, 800 Yale Blvd., N. E., Albuquerque, New Mexico 87131

Deidre A. Hunter AND Jon A. Holtzman

Lowell Observatory, 1400 W. Mars Hill Rd., Flagstaff, Arizona 86001

TOD R. LAUER

Kitt Peak National Observatory, National Optical Astronomy Observatories, ${ }^{2}$ P. O. Box 26732, Tucson, Arizona 85726

EDWARD J. SHAYA

Department of Physics, University of Maryland, College Park, Maryland 20742

ARTHUR CODE

Washburn Observatory, University of Wisconsin, 475 N. Charter St., Madison, Wisconsin 53706

S. M. FABER

Lick Observatory, University of California, Santa Cruz, California 95064

EDWARD J. GROTH

Physics Department, Princeton University, P.O. Box 708, Princeton, New Jersey 08544

ROBERT M. LIGHT

Lick Observatory, University of California, Santa Cruz, California 95064

ROGER LYNDS AND EARL J. O'NEIL, JR.

Kitt Peak National Observatory, National Optical Astronomy Observatories, ${ }^{2}$ P. O. Box 26732, Tucson, Arizona 85726

JAMES A. WESTPHAL

Division of Geological and Planetary Sciences, California Institute of Technology, Pasadena, California 91125 Received 14 May 1992; revised 24 July 1992

\begin{abstract}
The Planetary Camera of the Hubble Space Telescope has been used to obtain broad and narrowband images of R136, the core of the massive star cluster 30 Doradus in the Large Magellanic Cloud. R136a, the brightest component of $\mathrm{R} 136$, is shown to have at least 12 separate components, including the eight originally identified by speckle interferometry. Three of the 12 components are previously unidentified close companions of the speckle components. The stars within R136a are found to have luminosities and colors of normal evolved (Wolf-Rayet and blue supergiants) and zero-age main-sequence (ZAMS) massive stars. A narrowband $\mathrm{He}$ II filter was used to investigate the Wolf-Rayet stellar population. We find that three stars in R136a are of the Wolf-Rayet type; of the two identified from ground-based data, one is now resolved into two components. We present color-magnitude diagrams and a luminosity function of the stars within the larger region $(\sim 2 \mathrm{pc})$ defined as R136. We find that the stars in R136 are similar in color and luminosity to those of cluster members that lie outside that crowded inner region. The lower end of the color-magnitude diagram corresponds to ZAMS spectral type B3. No red supergiants have been detected within R136. The luminosity per unit area in the inner $1^{\prime \prime}(0.25 \mathrm{pc})$ of $\mathrm{R} 136$ is $\geqslant 50$ times that of the center of Orion for a comparable area and seven times that of the core of NGC 3603. The luminosity per unit area of all of R136 is comparable to that of Orion but is sustained over 130 times the area. An F336W surface brightness profile is constructed for R136 based on the stellar photometry. The distribution is found to be consistent with a pure power law with $I(r) \propto r^{\gamma}$ with $\gamma=-1.72 \pm 0.06$ or with a small core with $r_{c}<0.25^{\prime \prime}$, considerably smaller than predicted from ground-based observations. In the latter case $\rho_{0}>5 \times 10^{4} \mathscr{M}_{\odot} \mathrm{pc}^{-3}$. The implied upper limit on the relaxation time for the cluster is much smaller than the age of $3.5 \times 10^{6}$ yrs required by the presence of Wolf-Rayet stars. This suggests that relaxation effects have been very important in determining the observed structure of the cluster unless a large population of lower mass stars is also present.
\end{abstract}




\section{INTRODUCTION}

The 30 Doradus Nebula in the Large Magellanic Cloud (LMC) is a spectacular example of a supergiant $\mathrm{H}$ II region, near enough and sufficiently free of Galactic reddening so that most of the stellar constituents of the inner core can be resolved. Fully one quarter of the total massive star formation of the $\mathrm{LMC}$ is contained in a $15^{\prime}$ radius around 30 Doradus (Kennicutt 1991). The central region of the nebula is dominated by the cluster NGC 2070, which itself contains numerous very tight clusters of extremely massive OB stars and an unusually rich grouping of Wolf-Rayet stars, most of which are classified WN from the emission lines of highly ionized nitrogen appearing in their optical spectra. The most luminous of the 30 Doradus clusters in R136 (HD 38268), whose brightest component R136a has a spectral identification of O3If in both the UV and nearinfrared, and WN4.5-5+OB $(n)$ in the optical (Melnick 1985). This component was once believed to be a single star with a mass $>10^{3} \mathscr{M}_{\odot}$ (Cassinelli et al. 1981). This suggestion was based in part on the pointlike appearance of R136a in the optical end and UV ( $\left.<3^{\prime \prime}\right)$ and on the perceived difficulty of having as many as $30 \mathrm{O} 3$ stars within a $<1 \mathrm{pc}$ region as required to match the observed luminosity and inferred ionization (Savage et al. 1983).

Speckle interferometric observations (Weigelt \& Baier 1985; Neri \& Grewing 1988) and later Hubble Space Telescope (HST) Faint Object Camera images (Weigelt et al. 1991) resolved R136a into a minimum of eight components (R136a1-a8), all within 1", but spectral classifications for these individual objects have not as yet been made. The extreme core concentration of very early-type stars around R136a, however, along with features in R136a's composite spectrum, make it likely that the nonWolf-Rayet components in R136a itself are at least as early as O5. In addition to the apparent extreme density of earlytype stars, the difficulty which originally drove the supermassive star interpretation was the apparent deficit in ionizing radiation for the nebula as a whole. This has been addressed more recently by Melnick (1985) and Moffat $e t$ al. (1985) who show that the early $O$ and Wolf-Rayet stars within $2^{\prime}-3^{\prime}$ of the R136a core (but excluding R136a itself) can easily account for half the nebular ionization, requiring that as few as six early $O$ stars be present in R136a.

The extreme concentration of very high mass ( $>20$ $\mathscr{M}_{\odot}$ ) stars make the core of 30 Doradus a useful laboratory for massive star formation. In addition the large numbers of Wolf-Rayet and early-O spectra types in and surrounding $R 136$ form a unique case study of the role played by Wolf-Rayet stars in massive stellar evolution. Observations which could define the luminosity (and, by inference, the mass) function in and around the core of 30 Doradus

${ }^{1}$ Based on observations with the NASA/ESA Hubble Space Telescope, obtained at the Space Telescope Science Institute, which is operated by AURA, Inc., under NASA contract NAS 5-26555.

${ }^{2}$ The National Optical Astronomy Observatories are operated by the Association of Universities for Research in Astronomy, Inc., under a cooperative agreement with the National Science Foundation.
TABLE 1. The HST PC observations.

\begin{tabular}{ccccr}
\hline \hline date & filter & No. images & Exposure Time (s) & HST Image Name \\
\hline $8 / 25 / 90$ & F368M & 5 & $4 @ 300,1 @ 150$ & w0ce0102B \\
$8 / 25 / 90$ & F547M & 5 & $5 @ 100$ & w0ce0107B \\
$4 / 12 / 91$ & F36W & 1 & 80 & w0ia0104t \\
$4 / 12 / 91$ & F469N & 1 & 140 & w0ia0103t \\
$4 / 11 / 91$ & F555W & 1 & 16 & w0ia0101t \\
$4 / 12 / 91$ & F702W & 1 & 26 & w0ia0102t \\
\hline \hline
\end{tabular}

have heretofore been limited by dynamic range of detecting faint stars and by crowding among the very brightest stars.

Therefore, in order to examine the stellar content of the heart of this extraordinary star cluster, we obtained images of R136 with the HST Planetary Camera (PC), which has a pixel scale of $0.044^{\prime \prime}$. Although photometry of individual stars is compromised by the spherical aberration of the primary mirror of the telescope, the high resolution allowed by the sharp core of the point spread function enables one to identify stellar components to an extent not attainable from the ground. Furthermore, stellar photometry is possible, although the uncertainties are larger than they otherwise would have been without aberration. Early images of R136 were obtained through filters F368M and F547M, and a later set of observations were made with filters F336W, F555W, F702W, and F469N. Photometry of stars on the broadband images is used below to construct a color-magnitude diagram and luminosity function. The F469N filter is a narrowband filter that passes the emission-line He II $\lambda$ 44686, which is a characteristic of Wolf-Rayet stars. Therefore, the F469N image is used below to identify Wolf-Rayet stars. We take the distance modulus to the LMC to be -18.6 and the total reddening to 30 Doradus to be $\mathrm{E}(B-V)=0.34$ (Fitzpatrick \& Savage 1984).

\section{OBSERVATIONS}

\subsection{Data Obtained in 1990}

HST PC observations of 30 Doradus were taken as part of an evaluative program instituted shortly after the discovery of spherical aberration in the primary mirror. The core of the cluster was placed in the $800 \times 800$ CCD known as PC6 on 25 August 1990. [Details about the instrument can be found in Griffiths (1989)]. Observations were made through filters F368M and F547M. Although later data taken after UV-flooding and better calibration were used for the stellar photometry presented in this paper (see Sec. 2.2), we use the summed F368M image to illustrate the morphology of the stellar content and distribution. The F368M image is of higher signal-to-noise than the F336W image taken later, and it is on this image that we tried deconvolution. The F547M data also obtained, calibrated, and analyzed in 1990 are not presented here. The observations are listed in Table $I$.

Five exposures were made through filter F368M over two orbits: four at $300 \mathrm{~s}$ each and one at $150 \mathrm{~s}$. The multiple exposures were aligned (each shifted by less than 0.2 pixels) with a sinc interpolation, cosmic rays were flagged and removed, and the images were summed. Flat-fielding was problematical due to the lack of on-orbit flats for 
F368M and the fact that the detector had not been UVflooded. A flat obtained during prelaunch thermal vacuum tests in filter F336W was used, and it provided a relatively clean response correction, with excess noise apparent only in the extreme corners of each F368M frame.

In Fig. 1 (Plate 114) one can see the F368M image of the entire PC6 field and of the cluster center. Figure 2 (Plate 115) shows the F368M image displayed to show the individual stars in R136a. The first eight stars are numbered in the same way that Weigelt \& Baier (1985) numbered the speckle components. The rest of the stars continue this numbering scheme. The results of deconvolution of the cluster region, using the method of Lucy (1974) with 100 iterations, are also shown in Fig. 2. The deconvolution produced no stellar features which are not already visible in the original images.

\subsection{Data Obtained in 1991}

PC images of R136 were obtained on 11 and 12 April 1991 through broadband filters F336W, F555W, and F702W and through the narrowband filter F469N. Exposure times were $80,16,26$, and $140 \mathrm{~s}$, respectively. The observations are listed in Table I. R136 itself as placed near the center of PC6; other stars near the center of 30 Doradus fell on chips PC5, 7, and 8 as well. The images were corrected for A-D conversion errors, preflash, bias, and dark current. On-orbit streak flats collected from January to April 1991 were used to flat-field the CCD's. The streak flats had many problems, but they did a reasonable job of removing pixel-to-pixel and chip-to-chip variations. However, flat-fielding problems are another major limitation on the quality of the photometry (see WF/PC Investigation Definition Team 1992). The field of view of the PC (all four CCD's) is outlined in Fig. 3 (Plate 116) on a groundbased $V$-band image of this region kindly loaned to the WF/PC team. In Fig. 4 is displayed a mosaic of the four CCD images in filter F555W.

Measurements of the brightnesses of the stars in the broadband filters were made using the crowded-field photometry package DAOPHOT (Stetson 1987). The point spread function (PSF) varies significantly across the field of view of a PC chip. Because the PSF must be determined from stars outside the core of the cluster, the variable PSF is another fundamental limitation on the accuracy of the photometry of the stars in R136. A third difficulty comes from the extended halos of the stars; the measurable PSF extends to $\sim 60$ pixels in radius.

In addition to wreaking havoc with photometric determinations, the tendrils of the stellar halos often lead automatic routines for finding stars to falsely identify knots in the tendrils as stars. Therefore, in order to produce a clean list of stars, we examined the stars in the original coordinate list by eye to verify their reality on the F702W and F555W images. We retained only stars that appeared in the photometry lists of both frames. We also modified a parameter in DAOPHOT so that during the fitting procedure it would be more difficult for stars to be eliminated than in the standard software. Furthermore, for the dozen stars in the very core of $\mathrm{R} 136$, we made it even more difficult for DAOPHOT to toss out a star. Even so, however, star number 9 (see below) was not retained in the fitting procedure of F336W.

The DAOPHOT software returns several measures of the uncertainty of the photometry and goodness of fit. These parameters were used to eliminate objects as well. In particular stars with $\sigma>0.6$ were deleted from the photometry lists. This has the desired effect of eliminating cosmic rays and dubious star detections although it also eliminates legitimate stars at the faint end.

Because this clean star list was compiled essentially "by hand," it was not feasible to estimate the incompleteness of this list. To try to get a better estimate of the luminosity function, we created another star list entirely automatically, but not searching as faint in the frame. This avoided most of the problems with spurious detections. For the automatic list, we still required that objects be detected on both the F555W and F702W frames. We used the automatic technique to estimate completeness corrections as discussed below in the section on the luminosity function (Sec. 3.3).

The photometric zero points for F336W and F555W were taken from observations of the HST standard star field in Omega Cen on 2 April 1991. Since the dates of the R136 and Omega Cen observations are fairly close, the apparent zero point automatically corrects for any contamination in the optical path. This contamination increases with time after the CCD's are cooled and has the effect of decreasing the effective quantum efficiency. This is especially noticeable in the ultraviolet, where the zero point can change by tenths of a magnitude over time (see WF/PC Investigation Definition Team 1992). For F702W the 3 January 1991 measurement of the zero point was used and corrected by $0.01 \mathrm{mag}$ to allow for the contamination over the intervening three months to the R136 observations.

Note that, although the broadband filters are analogs to the Johnson-Cousins UVR filters, they are not identical. Thus, the magnitudes quoted below are on the WF/PC filter system which is close to, but not equal to, the Johnson-Cousins magnitudes. We have included a zero point, as described above, that ties the flight magnitudes to those of the ground-based system, but we have not included any color term. Note also that the transformations to the Johnson system given by Harris et al. (1991) do not apply to stars as blue as $\mathrm{O}$ and $\mathrm{B}$ stars and that intrinsic colors of massive stars in the WF/PC filter system are not currently known. In fact, to our knowledge, this is the first study of massive stars through WF/PC filters. We expect, however, that $\mathrm{F555W}$ is fairly close, i.e., within our photometric uncertainties, to a $V$ magnitude, and we will use it as an approximate $V$ magnitude in computing luminosities below.

The stars were sorted into those within R136 and those outside. R136 is defined as a box $7.3^{\prime \prime} \times 8.7^{\prime \prime}$ which very roughly approximates the ground-based definition of $\mathrm{R} 136$. In R136 we measured 214 stars. Outside of R136 we measured 800 stars on the four CCD's. The photometry for 


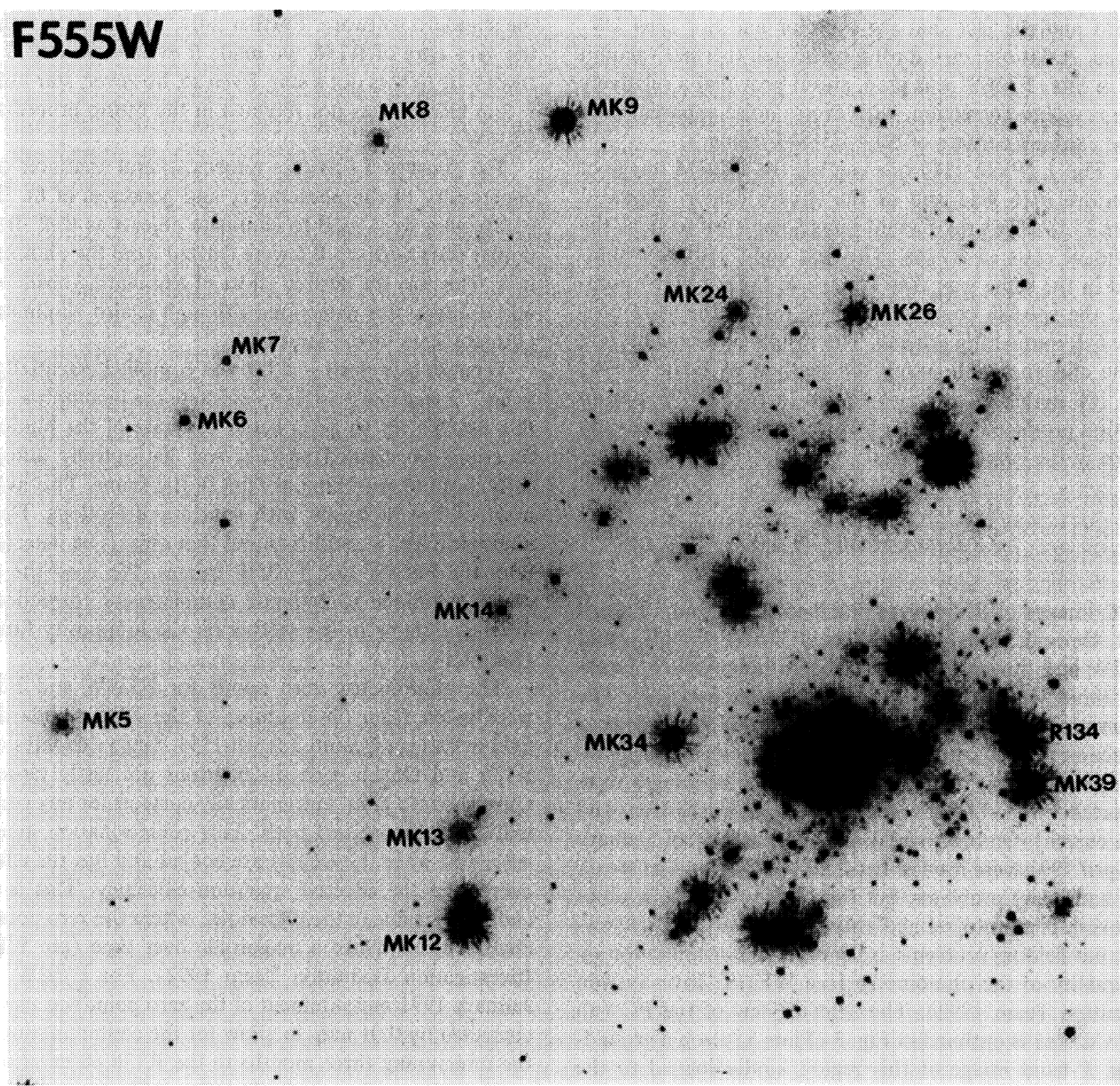

FIG. 4. A mosaic of the four PC CCD images is shown for the F555W filter. Stars not identified in Fig. 1 are identified here. Note that the individual PC images have been variously rotated in order to match the orientation on the sky.

stars in the box defined as R136 is presented in Table 2. Photometry for the rest of the stars is given in Tables 3 through 6 , where the star number begins with a $5,6,7$ or 8 to identify it as being found on PC5, PC6, PC7, or PC8, respectively. Figure 5 shows the uncertainty in the magnitudes as a function of magnitude for the F555W filter. Naturally, photometry of the more crowded R136 stars is more uncertain than that of stars outside R136 at a given magnitude level. However, the photometric uncertainties given by DAOPHOT and shown in the figure are undoubtedly an underestimate of the true uncertainties. Other sources of uncertainty include the zero point $(\sim 0.05 \mathrm{mag})$ and the flat fielding $(\sim 0.08 \mathrm{mag}$; see WF/PC Investigation Definition Team 1992).

The $x, y$ positions given in Tables 2-6 are the original pixel coordinates on the F702W frame. That is, the origin
0,0 of each chip is the pyramid corner of the Planetary Camera. Figure 3 shows the orientation of the field of view with respect to the sky. The center of the box outlined in Fig. 3 corresponds to the coordinate of the chip pixel given in Tables 2-6 that is both outside the pyramid overlap region and closest to the 0,0 coordinate of each chip. The box in Fig. 3 has a position angle of $25^{\circ}$, with PC5 to the lower left and PC's 6, 7, 8 following counterclockwise. In Table 7 a match is made between star number and alternate designations in the literature. These stars are also identified on Figs. 1 and 4 and can be used to orient the coordinate system of the tables with respect to known stars.

A 140 s image of R136 was also obtained through the narrowband filter F469N. This filter is useful for isolating the stellar emission-line feature He II $\lambda 4686$ that is characteristic of Wolf-Rayet stars. The stellar continuum also 
TABLE 2. Photometry of stars in R136.

\begin{tabular}{|c|c|c|c|c|c|c|c|c|c|c|c|c|c|}
\hline ID & $x$ & y & F555W & $\sigma$ & $336-555$ & $555-702$ & ID & $x$ & $\mathrm{y}$ & F555W & $\sigma$ & $336-555$ & $555-702$ \\
\hline 1 & 346.4 & 466.1 & 12.78 & 0.10 & -1.46 & 0.58 & 41 & 313.8 & 484.0 & 14.59 & 0.06 & -1.02 & 0.22 \\
\hline 2 & 344.3 & 464.2 & 13.09 & 0.10 & -0.89 & 0.40 & 42 & 362.6 & 382.3 & 14.60 & 0.04 & -0.59 & 0.41 \\
\hline 3 & 356.5 & 469.1 & 12.87 & 0.04 & -0.80 & 0.31 & 43 & 375.0 & 437.6 & 14.61 & 0.08 & -1.00 & 0.26 \\
\hline 4 & 342.3 & 474.3 & 13.56 & 0.07 & -0.85 & 0.16 & 44 & 352.7 & 442.9 & 14.70 & 0.07 & -0.87 & 0.18 \\
\hline 5 & 340.4 & 463.4 & 13.73 & 0.07 & -0.66 & 0.21 & 45 & 293.1 & 456.2 & 14.79 & 0.05 & -0.87 & 0.25 \\
\hline 6 & 361.2 & 472.2 & 14.06 & 0.12 & -0.78 & 0.35 & 46 & 349.0 & 443.9 & 14.90 & 0.08 & -0.74 & 0.13 \\
\hline 7 & 338.3 & 467.2 & 13.78 & 0.06 & -0.85 & 0.23 & 47 & $\mathbf{3 8 7 . 3}$ & 448.5 & 14.91 & 0.06 & -0.95 & 0.19 \\
\hline 8 & 341.3 & 472.6 & 14.28 & 0.11 & -1.20 & 0.25 & 48 & 280.8 & 388.2 & 14.92 & 0.03 & -0.80 & 0.23 \\
\hline 9 & 345.7 & 467.5 & 12.85 & 0.10 & - & 0.00 & 49 & 366.4 & 446.0 & 14.95 & 0.07 & -0.80 & 0.26 \\
\hline 10 & 354.1 & 472.0 & 14.51 & 0.10 & -1.05 & 0.25 & 50 & 335.3 & 499.1 & 14.98 & 0.10 & -0.74 & 0.09 \\
\hline 11 & 360.1 & 473.3 & 13.58 & 0.09 & -0.84 & 0.23 & 51 & 332.6 & 497.8 & 15.00 & 0.08 & -1.32 & 0.08 \\
\hline 12 & 335.9 & 469.1 & 14.68 & 0.10 & -0.72 & 0.08 & 52 & 359.9 & 440.8 & 15.01 & 0.10 & -0.86 & 0.21 \\
\hline 13 & 338.2 & 477.2 & 13.92 & 0.06 & -1.01 & 0.33 & 53 & 336.8 & 445.0 & 15.08 & 0.11 & -0.90 & 0.04 \\
\hline 14 & 350.6 & 482.3 & 15.18 & 0.07 & -0.72 & 0.10 & 54 & 388.4 & 431.9 & 15.14 & 0.11 & -0.95 & 0.10 \\
\hline 15 & 353.5 & 458.2 & 15.06 & 0.09 & -1.17 & 0.24 & 55 & 376.2 & 448.8 & 15.14 & 0.11 & -0.84 & 0.19 \\
\hline 16 & 360.1 & 461.9 & 14.72 & 0.07 & -0.79 & 0.14 & 56 & 301.3 & 527.3 & 15.19 & 0.05 & -1.10 & 0.18 \\
\hline 17 & 365.4 & 464.5 & 15.12 & 0.09 & -0.94 & 0.16 & 57 & 386.0 & 465.0 & 15.25 & 0.09 & -1.11 & 0.14 \\
\hline 18 & 361.8 & 458.6 & 15.95 & 0.17 & -1.03 & 0.31 & 58 & 385.4 & 430.1 & 15.29 & 0.09 & -0.92 & 0.21 \\
\hline 19 & 335.5 & 458.6 & 14.66 & 0.06 & -0.80 & 0.22 & 59 & 396.1 & 471.1 & 15.36 & 0.08 & -1.00 & 0.05 \\
\hline 20 & 332.7 & 463.0 & 14.58 & 0.07 & -0.84 & 0.02 & 60 & 324.5 & 457.2 & 15.38 & 0.09 & -0.93 & 0.23 \\
\hline 21 & 357.3 & 484.2 & 15.05 & 0.06 & -0.76 & 0.09 & 61 & 380.8 & 476.7 & 15.41 & 0.09 & -1.08 & -0.05 \\
\hline 22 & 362.8 & 464.9 & 15.36 & 0.14 & -0.75 & -0.24 & 62 & 323.1 & 434.0 & 15.41 & 0.16 & -0.87 & 0.16 \\
\hline 23 & 339.7 & 484.3 & 14.15 & 0.05 & -1.04 & 0.22 & 63 & 323.1 & 489.2 & 15.47 & 0.12 & -1.20 & 0.18 \\
\hline 24 & 335.5 & 486.5 & 15.20 & 0.08 & -1.04 & 0.28 & 64 & 331.8 & 507.1 & 15.49 & 0.09 & -0.99 & 0.14 \\
\hline 25 & 359.9 & 392.0 & 12.86 & 0.05 & -0.53 & 0.43 & 65 & 329.2 & 492.1 & 15.53 & 0.10 & -0.90 & 0.34 \\
\hline 26 & 346.6 & 419.3 & 13.03 & 0.04 & -0.71 & 0.36 & 66 & 390.0 & 455.2 & 15.53 & 0.09 & -0.75 & -0.03 \\
\hline 27 & 373.2 & 442.2 & 13.83 & 0.06 & -0.86 & 0.16 & 67 & 322.7 & 470.9 & 15.55 & 0.10 & -1.05 & 0.19 \\
\hline 28 & 311.8 & 517.5 & 14.20 & 0.05 & -1.11 & 0.14 & 68 & 263.5 & 398.7 & 15.55 & 0.05 & -0.79 & 0.40 \\
\hline 29 & 330.7 & 436.3 & 14.21 & 0.05 & -0.84 & 0.36 & 69 & 418.3 & 395.9 & 15.62 & 0.06 & -0.88 & 0.10 \\
\hline 30 & 322.6 & 436.3 & 14.26 & 0.07 & -0.75 & 0.19 & 70 & 333.9 & 492.8 & 15.64 & 0.13 & -0.81 & 0.12 \\
\hline 31 & 307.2 & 473.8 & 14.28 & 0.05 & -1.00 & 0.1 & 71 & 395.9 & 487.8 & 15.66 & 0.06 & -1.07 & 0.10 \\
\hline 32 & 347.7 & 449.9 & 14.30 & 0.06 & -0.78 & 0.32 & 72 & 321.1 & 496.1 & 15.66 & 0.11 & -0.98 & 0.04 \\
\hline 33 & 306.3 & 482.2 & 14.31 & 0.05 & -1.03 & 0.16 & 73 & 370.2 & 419.2 & 15.66 & 0.10 & - & 0.42 \\
\hline 34 & 345.2 & 506.4 & 14.32 & 0.06 & -0.99 & 0.19 & 74 & 415.8 & 432.2 & 15.69 & 0.06 & -0.67 & 0.16 \\
\hline 35 & 334.3 & 417.5 & 14.37 & 0.05 & -0.81 & 0.12 & 75 & 364.1 & 479.2 & 15.69 & 0.14 & - & -0.19 \\
\hline 36 & 333.5 & 442.3 & 14.47 & 0.06 & -0.87 & 0.25 & 76 & 374.1 & 456.8 & 15.72 & 0.11 & -0.95 & 0.26 \\
\hline 37 & 321.3 & 373.1 & 14.52 & 0.04 & -0.74 & 0.28 & 77 & 315.9 & 471.6 & 15.72 & 0.10 & -0.82 & -0.09 \\
\hline 38 & 366.7 & 452.0 & 14.52 & 0.06 & -1.09 & 0.14 & 78 & 300.0 & 502.8 & 15.76 & 0.08 & -0.95 & 0.24 \\
\hline 39 & 331.7 & 413.9 & 14.53 & 0.05 & -0.89 & 0.24 & 79 & 329.3 & 456.2 & 15.77 & 0.13 & -0.56 & 0.04 \\
\hline 40 & 312.0 & 447.9 & 14.54 & 0.06 & -0.87 & 0.26 & 80 & 383.6 & 505.6 & 15.78 & 0.08 & -1.01 & -0.07 \\
\hline
\end{tabular}

(b)

\begin{tabular}{|c|c|c|c|c|c|c|c|c|c|c|c|c|c|}
\hline ID & $\mathrm{x}$ & $\mathrm{y}$ & F555W & $\sigma$ & $336-555$ & $555-702$ & ID & $x$ & $y$ & F555W & $\sigma$ & $336-555$ & $555-702$ \\
\hline 81 & 320.1 & 488.2 & 15.80 & 0.14 & -0.86 & 0.03 & 121 & 376.1 & 485.0 & 16.24 & 0.14 & -0.91 & -0.08 \\
\hline 82 & 323.2 & 462.1 & 15.81 & 0.12 & -0.75 & -0.19 & 122 & 293.7 & 422.7 & 16.28 & 0.09 & -0.81 & 0.22 \\
\hline 83 & 414.9 & 352.5 & 15.81 & 0.04 & -0.92 & 0.15 & 123 & 323.3 & 385.1 & 16.29 & 0.12 & -0.63 & 0.23 \\
\hline 84 & 388.0 & 546.0 & 15.82 & 0.06 & -0.87 & 0.11 & 124 & 341.5 & 401.5 & 16.31 & 0.10 & -0.80 & 0.16 \\
\hline 85 & 340.0 & 413.0 & 15.83 & 0.12 & -0.72 & 0.27 & 125 & 367.9 & 502.2 & 16.31 & 0.15 & -0.94 & 0.08 \\
\hline 86 & 289.9 & 440.6 & 15.84 & 0.08 & -0.76 & 0.30 & 126 & 397.3 & 460.8 & 16.32 & 0.13 & -1.06 & 0.03 \\
\hline 87 & 368.9 & 459.9 & 15.85 & 0.13 & -0.20 & 0.06 & 127 & 365.3 & 511.5 & 16.32 & 0.13 & -0.86 & 0.10 \\
\hline 88 & 322.2 & 403.0 & 15.86 & 0.09 & -0.76 & 0.33 & 128 & 369.6 & 455.9 & 16.35 & 0.21 & -1.16 & -0.50 \\
\hline 89 & 327.9 & 497.0 & 15.86 & 0.14 & -0.80 & 0.12 & 129 & 297.1 & 479.2 & 16.36 & 0.10 & -0.60 & 0.06 \\
\hline 90 & 263.5 & 482.6 & 15.87 & 0.05 & -0.73 & 0.13 & 130 & 411.2 & 477.3 & 16.37 & 0.07 & -0.79 & 0.20 \\
\hline 91 & 375.3 & 414.3 & 15.88 & 0.12 & -0.49 & 0.16 & 131 & 387.1 & 489.4 & 16.39 & 0.14 & -0.90 & 0.13 \\
\hline 92 & 272.5 & 353.4 & 15.89 & 0.04 & -0.55 & 0.21 & 132 & 363.2 & 373.7 & 16.44 & 0.08 & -0.58 & 0.33 \\
\hline 93 & 364.2 & 491.4 & 15.89 & 0.13 & -0.86 & 0.30 & 133 & 264.5 & 380.8 & 16.44 & 0.05 & -0.80 & 0.24 \\
\hline 94 & 378.5 & 492.4 & 15.89 & 0.10 & -0.95 & 0.16 & 134 & 359.4 & 504.9 & 16.45 & 0.19 & -0.75 & 0.11 \\
\hline 95 & 275.8 & 413.7 & 15.91 & 0.05 & -0.73 & 0.31 & 135 & 372.3 & 473.4 & 16.45 & 0.19 & - & 0.24 \\
\hline 96 & 356.2 & 386.5 & 15.92 & 0.11 & -0.24 & 0.73 & 136 & 355.1 & 403.7 & 16.45 & 0.11 & -0.54 & 0.05 \\
\hline 97 & 349.9 & 413.7 & 15.93 & 0.10 & 0.24 & 0.14 & 137 & 304.9 & 444.3 & 16.48 & 0.15 & - & 0.13 \\
\hline 98 & 368.5 & 491.1 & 15.96 & 0.12 & -0.68 & 0.16 & 138 & 324.6 & 526.8 & 16.49 & 0.10 & -0.89 & 0.23 \\
\hline 99 & 309.8 & 451.2 & 15.98 & 0.17 & -0.85 & 0.02 & 139 & 351.9 & 410.1 & 16.51 & 0.16 & -1.08 & 0.46 \\
\hline 100 & 411.8 & 496.7 & 15.99 & 0.06 & -1.21 & 0.35 & 140 & 330.2 & 525.9 & 16.52 & 0.11 & -0.66 & 0.12 \\
\hline 101 & 285.8 & 491.9 & 15.99 & 0.06 & -0.85 & 0.15 & 141 & 352.0 & 499.2 & 16.53 & 0.23 & -0.86 & 0.17 \\
\hline 102 & 380.3 & 384.6 & 16.04 & 0.06 & -0.68 & 0.11 & 142 & 304.4 & 458.1 & 16.55 & 0.19 & - & -0.02 \\
\hline 103 & 304.5 & 424.9 & 16.05 & 0.10 & - & 0.15 & 143 & 279.9 & 395.1 & 16.56 & 0.07 & -0.80 & 0.09 \\
\hline 104 & 354.9 & 376.7 & 16.05 & 0.08 & -0.87 & 0.31 & 144 & 390.8 & 501.8 & 16.61 & 0.12 & -1.14 & 0.11 \\
\hline 105 & 319.1 & 451.6 & 16.05 & 0.18 & -0.71 & 0.35 & 145 & 385.3 & 394.8 & 16.63 & 0.10 & -0.69 & 0.18 \\
\hline 106 & 363.5 & 454.9 & 16.06 & 0.19 & - & 0.05 & 146 & 362.9 & 366.0 & 16.66 & 0.08 & -0.78 & 0.44 \\
\hline 107 & 379.6 & 484.7 & 16.06 & 0.12 & -1.24 & 0.24 & 147 & 413.8 & 393.3 & 16.67 & 0.09 & -0.87 & 0.33 \\
\hline 108 & 374.1 & 408.8 & 16.08 & 0.10 & -0.75 & 0.33 & 148 & 372.2 & 495.6 & 16.70 & 0.18 & - & 0.20 \\
\hline 109 & 301.5 & 486.3 & 16.08 & 0.12 & -1.01 & 0.22 & 149 & 324.3 & 483.0 & 16.70 & 0.22 & - & 0.52 \\
\hline 110 & 264.4 & 478.6 & 16.09 & 0.06 & -1.09 & 0.26 & 150 & 359.7 & 404.9 & 16.75 & 0.15 & - & 0.19 \\
\hline 111 & 390.5 & 431.2 & 16.09 & 0.19 & -0.63 & -0.04 & 151 & 363.0 & 486.6 & 16.77 & 0.29 & - & 0.32 \\
\hline 112 & 284.2 & 460.0 & 16.11 & 0.08 & -1.03 & 0.24 & 152 & 363.6 & 503.6 & 16.81 & 0.22 & -1.11 & -0.08 \\
\hline 113 & 337.7 & 513.3 & 16.11 & 0.13 & -0.70 & 0.07 & 153 & 372.2 & 491.0 & 16.82 & 0.21 & - & -0.42 \\
\hline 114 & 361.1 & 487.8 & 16.12 & 0.17 & -0.33 & 0.11 & 154 & 290.1 & 479.0 & 16.91 & 0.12 & -0.86 & 0.08 \\
\hline 115 & 349.7 & 458.3 & 16.13 & 0.21 & - & 0.00 & 155 & 323.0 & 512.1 & 16.95 & 0.20 & - & 0.22 \\
\hline 116 & 316.5 & 448.2 & 16.15 & 0.18 & -0.96 & 0.06 & 156 & 392.1 & 542.4 & 16.99 & 0.08 & -0.49 & 0.08 \\
\hline 117 & 317.1 & 480.1 & 16.19 & 0.18 & -0.35 & 0.18 & 157 & 384.8 & 362.6 & 16.99 & 0.11 & - & 0.28 \\
\hline 118 & 313.6 & 403.0 & 16.19 & 0.09 & -0.89 & 0.30 & 158 & 378.3 & 397.3 & 17.08 & 0.17 & -0.01 & 0.17 \\
\hline 119 & 288.1 & 469.8 & 16.20 & 0.08 & -0.93 & 0.14 & 159 & 272.5 & 483.3 & 17.09 & 0.11 & -0.82 & 0.21 \\
\hline 120 & 348.5 & 499.3 & 16.22 & 0.18 & -1.01 & 0.28 & 160 & 395.6 & 397.3 & 17.11 & 0.15 & - & 0.15 \\
\hline
\end{tabular}


TABLE 2. (continued)

\begin{tabular}{|c|c|c|c|c|c|c|c|c|c|c|c|c|c|}
\hline ID & $x$ & $\mathrm{y}$ & F555W & $\sigma$ & $336-555$ & $555-702$ & ID & $x$ & $y$ & F555W & $\bar{\sigma}$ & $336-555$ & $555-702$ \\
\hline 161 & 288.4 & 497.0 & 17.11 & 0.15 & -0.61 & 0.25 & 188 & 422.5 & 524.7 & 17.68 & 0.08 & -0.74 & 0.18 \\
\hline 162 & 282.2 & 444.7 & 17.11 & 0.14 & -1.24 & 0.10 & 189 & 410.0 & 460.0 & 17.75 & 0.20 & -0.90 & 0.01 \\
\hline 163 & 410.2 & 499.6 & 17.12 & 0.16 & -0.12 & -0.59 & 190 & 405.1 & 536.7 & 17.77 & 0.11 & - & 0.21 \\
\hline 164 & 309.0 & 414.1 & 17.16 & 0.20 & -1.19 & -0.01 & 191 & 402.2 & 412.4 & 17.80 & 0.23 & -0.75 & 0.58 \\
\hline 165 & 380.9 & 540.2 & 17.16 & 0.08 & -0.63 & -0.38 & 192 & 291.2 & 353.9 & 17.89 & 0.09 & - & 0.10 \\
\hline 166 & 287.7 & 464.3 & 17.18 & 0.17 & -0.81 & 0.12 & 193 & 277.4 & 510.9 & 17.90 & 0.19 & -0.36 & -0.42 \\
\hline 167 & 304.5 & 531.2 & 17.18 & 0.16 & - & -0.26 & 194 & 417.1 & 448.9 & 17.90 & 0.18 & -0.74 & -0.09 \\
\hline 168 & 310.4 & 438.5 & 17.19 & 0.24 & -0.90 & 0.29 & 195 & 406.6 & 541.2 & 17.94 & 0.12 & - & -0.08 \\
\hline 169 & 279.1 & 508.6 & 17.20 & 0.12 & -0.96 & 0.21 & 196 & 354.5 & 542.2 & 17.94 & 0.14 & - & 0.30 \\
\hline 170 & 266.9 & 519.4 & 17.24 & 0.08 & -0.76 & 0.18 & 197 & 355.6 & 535.3 & 17.98 & 0.20 & - & 0.10 \\
\hline 171 & 311.9 & 512.5 & 17.25 & 0.32 & - & 0.40 & 198 & 270.7 & $400: 8$ & 18.01 & 0.16 & - & 0.83 \\
\hline 172 & 409.1 & 469.1 & 17.30 & 0.14 & - & 0.13 & 199 & 408.8 & 390.1 & 18.04 & 0.17 & - & 0.29 \\
\hline 173 & 388.9 & 518.1 & 17.33 & 0.14 & - & 0.12 & 200 & 425.5 & 468.5 & 18.04 & 0.16 & -1.04 & 0.37 \\
\hline 174 & 292.2 & 488.8 & 17.34 & 0.20 & -0.52 & 0.42 & 201 & 412.1 & 508.0 & 18.17 & 0.15 & - & 0.16 \\
\hline 175 & 273.2 & 415.6 & 17.38 & 0.14 & -1.64 & 0.13 & 202 & 375.3 & 532.3 & 18.25 & 0.19 & -1.37 & 0.18 \\
\hline 176 & 302.7 & 456.0 & 17.38 & 0.32 & - & 0.21 & 203 & 268.9 & 384.0 & 18.30 & 0.18 & -1.20 & -0.15 \\
\hline 177 & 347.9 & 353.6 & 17.38 & 0.14 & - & 0.44 & 204 & 421.4 & 495.8 & 18.31 & 0.16 & - & 0.11 \\
\hline 178 & 284.5 & 468.4 & 17.39 & 0.18 & -0.24 & 0.01 & 205 & 320.9 & 538.8 & 18.32 & 0.24 & -1.08 & 0.23 \\
\hline 179 & 405.4 & 493.5 & 17.44 & 0.20 & -1.55 & 0.40 & 206 & 272.1 & 521.6 & 18.40 & 0.16 & -1.34 & 0.59 \\
\hline 180 & 279.9 & 475.0 & 17.46 & 0.17 & - & 0.10 & 207 & 423.9 & 403.6 & 18.44 & 0.21 & - & 0.27 \\
\hline 181 & 417.2 & 479.2 & 17.50 & 0.12 & -1.09 & 0.08 & 208 & 287.8 & 534.3 & 18.66 & 0.21 & - & 0.13 \\
\hline 182 & 411.1 & 450.4 & 17.51 & 0.17 & - & 0.03 & 209 & 264.8 & 536.4 & 18.72 & 0.19 & - & 0.41 \\
\hline 183 & 407.5 & 493.7 & 17.52 & 0.22 & - & 0.03 & 210 & 420.9 & 510.3 & 18.80 & 0.20 & - & 0.40 \\
\hline 184 & 276.5 & 482.0 & 17.61 & 0.17 & -0.29 & 0.24 & 211 & 344.8 & 538.5 & 18.86 & 0.39 & -1.25 & 0.59 \\
\hline 185 & 418.6 & 460.7 & 17.62 & 0.14 & -0.86 & 0.11 & 212 & 416.2 & 512.6 & 18.87 & 0.22 & - & 0.23 \\
\hline $186^{\circ}$ & 280.3 & 423.0 & 17.62 & 0.17 & - & 0.19 & 213 & 312.3 & 539.0 & 19.10 & 0.45 & - & 0.38 \\
\hline 187 & 358.5 & 533.1 & 17.63 & 0.13 & -0.94 & 0.06 & 214 & 280.8 & 521.7 & 19.21 & 0.40 & - & 0.56 \\
\hline
\end{tabular}

passed by the filter passband was approximated by a linear combination of the F336W and F555W images, and the continuum was subtracted from the F469N image. The resulting image shows those stars in which He II emission can be found. These stars and their magnitudes in the subtracted $\mathrm{F} 469 \mathrm{~N}$ image relative to that of star R134 are listed in Table 8. The PC6 F469N image itself is shown in Fig. 6 (Plate 117). The center of R136 is shown in Fig. 7.

\section{STELLAR CONTENT}

\subsection{Identification of Components}

We confirm what numerous studies have concluded before: that $\mathrm{R} 136 \mathrm{a}$ is not an unusual single star, but rather a tight collection of massive stars. R136a itself breaks up into 12 or more stars, depending on where you draw the boundary. These components include the 8 stars identified by Weigelt \& Baier (1985) from speckle interferometry. Of those 8 , stars 1,3 , and 6 are themselves clearly resolved as doubles in the PC images. In all we count 20 stars within the inner 1.5" centered on stars 1, 2, and 9 (see Fig. 2), and 214 stars in the box $(1.9 \times 2.2 \mathrm{pc})$ we use to define R136.

\subsection{The Color-Magnitude Diagram}

Before we can convert apparent magnitudes into absolute magnitudes we have to estimate the external and internal extinction of the starlight at the center of 30 Doradus. Fitzpatrick \& Savage (1984) examined several stars near the core of the cluster and concluded that the total reddening is $\mathrm{E}(B-V)=0.34$. In Fig. 8 we plot an (F336W-F555W), (F555W-F702W) color-color diagram. Also on the plot is a line indicating intrinsic UVR colors (Johnson 1968) although one must bear in mind that the HST colors have not been transformed to the Johnson system. Also shown on the plot is the reddening line for an $\mathrm{E}(B-V)$ of 0.34 . Extinction is known to be variable across the 30 Doradus region (cf. Melnick 1986) and in principle could even vary across the small field of view of the PC, particularly between the core R136 and stars external to it. However, because the intrinsic colors of massive stars in the WF/PC filter system are not known and because the photometric uncertainties are so large, we have simply adopted the reddening determined by Fitzpatrick and Savage and have applied it to all of the stars we measured. In the color-color diagram one can see that adopting Fitzpatrick and Savage's reddening will give a reasonable correction to the extinction for the bulk of the stars. The extinction in the WF/PC filters for a given $\mathrm{E}(B-V)$ is taken from Edvardsson \& Bell's (1989) work for low mass stars. Therefore, for all stars we adopt $A_{336}$ $=1.68, A_{555}=1.04$, and $A_{702}=0.68$.

In Figs. 9 and 10 we show color-magnitude diagrams. One can see that the stars in R136 cover the range of $M_{555}$ from about -7 down to about -1 . These limits correspond approximately to Wolf-Rayet or $\mathrm{O}$ supergiant type stars down to that of B3 ZAMS. This confirms suggestions that the stars in R136 in R136a are not unusual in terms of their individual luminosities. Furthermore, the stars in R136 and R136a are not even the brightest stars in the 30 Doradus region in the $\mathrm{F} 555 \mathrm{~W}$ filter.

The sequence on the $\left(M_{336}-M_{555}\right)_{0}$ vs $M_{555}$ plot is mostly vertical at about $\left(M_{336}-M_{555}\right)_{0}=-1.5$, reflecting the fact that we are looking at massive blue stars. The sequence is about $0.5 \mathrm{mag}$ wide, due in part to the large photometric uncertainties and possibly due also to variable 
TABLE 3. Photometry of stars outside R136 (PC5).

\begin{tabular}{|c|c|c|c|c|c|c|c|c|c|c|c|c|c|}
\hline ID & $x$ & $\bar{y}$ & F555W & $\bar{\sigma}$ & $336-555$ & $555-702$ & ID & $x$ & $\bar{y}$ & F555W & $\bar{\sigma}$ & $336-555$ & $555-702$ \\
\hline 5001 & 149.7 & 585.7 & 12.47 & 0.08 & -0.68 & 0.30 & 5041 & 550.6 & 705.0 & 17.86 & 0.08 & -0.66 & 0.26 \\
\hline 5002 & 152.1 & 543.7 & 14.07 & 0.08 & -0.91 & & 5042 & 299.7 & 608.1 & 17.93 & 0.11 & - & 0.34 \\
\hline 5003 & 724.5 & 279.5 & 14.09 & 0.00 & -0.98 & 0.20 & 5043 & 122.7 & 200.6 & 17.96 & 0.10 & - & 0.05 \\
\hline 5004 & 93.0 & 129.8 & 14.24 & 0.09 & -0.84 & 0.20 & 5044 & 651.1 & 606.0 & 18.00 & 0.07 & -0.70 & 0.23 \\
\hline 5005 & 127.7 & 423.6 & 15.30 & 0.08 & -1.04 & 0.19 & 5045 & 125.8 & 49.8 & 18.01 & 0.08 & -0.72 & 0.30 \\
\hline 5006 & 494.5 & 360.7 & 15.49 & 0.04 & -1.10 & 0.07 & 5046 & 139.8 & 460.5 & 18.09 & 0.33 & -1.90 & 0.52 \\
\hline 5007 & 287.7 & 407.1 & 16.06 & 0.05 & -1.03 & 0.14 & 5047 & 150.5 & 723.6 & 18.11 & 0.08 & - & 0.82 \\
\hline 5008 & 293.4 & 459.0 & 16.18 & 0.07 & 0.47 & 0.56 & 5048 & 192.2 & 793.1 & 18.14 & 0.09 & - & 0.29 \\
\hline 5009 & 78.1 & 775.0 & 16.19 & 0.08 & -1.01 & 0.32 & 5049 & 429.6 & 702.3 & 18.14 & 0.11 & -0.72 & 0.19 \\
\hline 5010 & 659.5 & 539.6 & 16.37 & 0.04 & -0.87 & 0.11 & 5050 & 75.4 & 656.1 & 18.19 & 0.09 & -0.40 & 0.41 \\
\hline 5011 & 84.2 & 439.1 & 16.49 & 0.11 & -1.01 & 0.19 & 5051 & 668.1 & 214.0 & 18.20 & 0.07 & -0.76 & 0.22 \\
\hline 5012 & 226.6 & 555.6 & 16.50 & 0.06 & -0.95 & 0.25 & 5052 & 487.3 & 289.0 & 18.26 & 0.09 & - & 0.64 \\
\hline 5013 & 494.2 & 255.3 & 16.52 & 0.04 & -1.02 & 0.09 & 5053 & 248.5 & 564.7 & 18.32 & 0.12 & - & 0.17 \\
\hline 5014 & 325.6 & 256.0 & 16.54 & 0.07 & -1.16 & 0.06 & 5054 & 268.9 & 386.2 & 18.35 & 0.11 & -0.76 & 0.15 \\
\hline 5015 & 433.9 & 791.0 & 16.67 & 0.06 & -0.73 & 0.26 & 5055 & 154.7 & 717.9 & 18.36 & 0.11 & -0.92 & 0.27 \\
\hline 5016 & 732.6 & 195.6 & 16.78 & 0.02 & -1.11 & 0.07 & 5056 & 306.5 & 220.8 & 18.44 & 0.12 & -1.03 & 0.22 \\
\hline 5017 & 462.7 & 735.8 & 16.85 & 0.07 & 0.44 & 0.60 & 5057 & 130.2 & 80.0 & 18.53 & 0.12 & - & 0.20 \\
\hline 5018 & 582.1 & 741.3 & 16.87 & 0.07 & - & -0.33 & 5058 & 548.1 & 84.5 & 18.56 & 0.09 & -0.55 & 0.28 \\
\hline 5019 & 395.1 & 691.8 & 16.90 & 0.07 & -1.05 & 0.22 & 5059 & 241.9 & 419.1 & 18.57 & 0.13 & -0.23 & 0.21 \\
\hline 5020 & 320.8 & 613.1 & 16.92 & 0.07 & -0.92 & 0.24 & 5060 & 496.0 & 108.2 & 18.63 & 0.10 & -0.47 & 0.10 \\
\hline 5021 & 208.0 & 586.3 & 16.98 & 0.08 & -0.78 & 0.25 & 5061 & 754.8 & 711.1 & 18.63 & 0.12 & -0.40 & 0.00 \\
\hline 5022 & 258.1 & 100.8 & 16.98 & 0.08 & -0.98 & 0.15 & 5062 & 127.0 & 65.4 & 18.66 & 0.12 & -0.40 & 0.19 \\
\hline 5023 & 304.8 & 608.9 & 16.99 & 0.07 & -0.94 & 0.33 & 5063 & 311.3 & 758.3 & 18.70 & 0.12 & - & 0.39 \\
\hline 5024 & 374.9 & 472.7 & 17.00 & 0.06 & -1.14 & 0.14 & 5064 & 75.2 & 355.0 & 18.72 & 0.14 & - & 0.06 \\
\hline 5025 & 133.7 & 430.7 & 17.04 & 0.15 & -1.45 & 0.13 & 5065 & 128.0 & 282.6 & 18.74 & 0.10 & -1.09 & -0.11 \\
\hline 5026 & 405.5 & 125.4 & 17.04 & 0.06 & -0.73 & 0.06 & 5066 & 160.2 & 741.3 & 18.74 & 0.13 & -0.57 & 0.17 \\
\hline 5027 & 148.0 & 552.2 & 17.10 & 0.36 & -2.13 & 0.18 & 5067 & 451.0 & 595.1 & 18.74 & 0.15 & -0.74 & 0.36 \\
\hline 5028 & 345.7 & 111.5 & 17.10 & 0.06 & -0.70 & 0.34 & 5068 & 569.1 & 710.9 & 18.77 & 0.14 & -0.96 & 0.16 \\
\hline 5029 & 114.8 & 221.6 & 17.14 & 0.07 & -0.79 & 0.46 & 5069 & 309.0 & 597.6 & 18.78 & 0.15 & - & 0.11 \\
\hline 5030 & 404.8 & 730.9 & 17.16 & 0.07 & -0.87 & 0.16 & 5070 & 467.9 & 276.3 & 18.81 & 0.12 & -0.58 & 0.41 \\
\hline 5031 & 82.2 & 369.5 & 17.22 & 0.08 & -1.00 & 0.19 & 5071 & 202.1 & 213.4 & 18.83 & 0.10 & - & 0.23 \\
\hline 5032 & 409.4 & 574.6 & 17.35 & 0.10 & -1.28 & 0.41 & 5072 & 670.2 & 419.4 & 18.86 & 0.10 & -0.71 & 0.37 \\
\hline 5033 & 639.6 & 536.2 & 17.38 & 0.05 & -0.85 & 0.26 & 5073 & 243.3 & 776.2 & 18.86 & 0.12 & -0.79 & 0.16 \\
\hline 5034 & 107.0 & 513.8 & 17.42 & 0.09 & -1.13 & 0.37 & 5074 & 363.0 & 722.2 & 18.88 & 0.16 & -0.62 & 0.22 \\
\hline 5035 & 73.7 & 773.5 & 17.49 & 0.16 & -0.04 & 0.00 & 5075 & 286.5 & 630.2 & 18.89 & 0.15 & - & 0.25 \\
\hline 5036 & 126.8 & 199.8 & 17.54 & 0.08 & -0.82 & 0.40 & 5076 & 471.6 & 281.3 & 18.91 & 0.13 & -0.87 & 0.08 \\
\hline 5037 & 144.5 & 164.6 & 17.57 & 0.09 & -0.68 & 0.29 & 5077 & 227.1 & 641.2 & 18.91 & 0.19 & -0.91 & -0.10 \\
\hline 5038 & 65.8 & 604.8 & 17.74 & 0.09 & -0.72 & 0.11 & 5078 & 385.7 & 523.8 & 18.96 & 0.13 & -1.26 & 0.44 \\
\hline 5039 & 51.6 & 248.0 & 17.75 & 0.08 & -0.95 & 0.25 & 5079 & 389.8 & 208.3 & 19.01 & 0.12 & - & 0.19 \\
\hline 5040 & 225.0 & 582.8 & 17.79 & 0.09 & -0.42 & 0.32 & 5080 & 618.3 & 333.0 & 19.02 & 0.12 & -0.77 & 0.08 \\
\hline
\end{tabular}

\begin{tabular}{rrrrrrrrrrrrrrr}
\hline ID & $\mathrm{x}$ & $\mathrm{y}$ & F555W & $\sigma$ & $336-555$ & $555-702$ & ID & $\mathrm{x}$ & $\mathrm{y}$ & F555W & $\sigma$ & $336-555$ & $555-702$ \\
\hline 5081 & 175.8 & 260.6 & 19.11 & 0.16 & -1.33 & -0.29 & 5093 & 236.0 & 170.6 & 19.57 & 0.22 & - & -0.05 \\
5082 & 187.9 & 63.0 & 19.13 & 0.17 & - & 1.38 & 5094 & 235.0 & 604.0 & 19.57 & 0.31 & - & 0.24 \\
5083 & 721.3 & 519.1 & 19.16 & 0.16 & - & 0.34 & 5095 & 60.4 & 566.6 & 19.59 & 0.26 & -1.48 & 0.82 \\
5084 & 290.9 & 392.8 & 19.20 & 0.21 & - & 0.20 & 5096 & 455.2 & 672.8 & 19.60 & 0.32 & - & 0.42 \\
5085 & 141.7 & 672.2 & 19.20 & 0.19 & - & 0.34 & 5097 & 320.3 & 296.8 & 19.70 & 0.34 & -1.35 & 0.59 \\
5086 & 229.2 & 545.3 & 19.22 & 0.23 & - & 0.13 & 5098 & 465.2 & 679.2 & 19.79 & 0.36 & - & 0.20 \\
5087 & 567.6 & 181.9 & 19.24 & 0.18 & - & 0.58 & 5099 & 524.7 & 608.5 & 19.86 & 0.21 & - & 0.52 \\
5088 & 328.0 & 365.1 & 19.27 & 0.22 & - & 0.14 & 5100 & 459.0 & 512.0 & 20.06 & 0.22 & - & 0.51 \\
5089 & 232.9 & 295.0 & 19.29 & 0.17 & - & -0.39 & 5101 & 323.4 & 782.7 & 20.15 & 0.34 & - & 0.80 \\
5090 & 204.3 & 353.8 & 19.33 & 0.19 & -1.51 & -0.04 & 5102 & 343.5 & 563.2 & 20.22 & 0.52 & - & 0.51 \\
5091 & 258.2 & 305.7 & 19.40 & 0.20 & - & 0.30 & 5103 & 284.6 & 316.0 & 20.58 & 0.48 & - & 1.27 \\
5092 & 621.1 & 501.8 & 19.48 & 0.21 & - & 0.16 & 5104 & 157.7 & 455.9 & 13.77 & 0.08 & -1.05 & 0.05 \\
\hline
\end{tabular}

reddening across the cluster. However, some spread is likely to be intrinsic since the relatively uncrowded stars outside R136 show the same spread as those within R136, where the photometric uncertainties are larger. Intrinsic spread could be due to the natural spread among massive stars as they evolve (Stothers \& Chin 1977; Schaller et al. 1992 ) and could be a signature of stars undergoing extreme mass loss and convective overshoot (Maeder \& Meynet 1987). At about $M_{555}=-2$ one may possibly see a clump of slightly redder stars, presumably as the main sequence begins to curve to the right for cooler, lower mass stars. Also, at $M_{555} \sim-6.5$ one begins to see a slight turn to the red. This shows up clearly as the main-sequence turnoff in the color-magnitude diagrams of the larger 30 Doradus region measured by Melnick (1986). On the $\left(M_{555}-M_{702}\right)$ vs $M_{702}$ plot, the sequence is narrower and does not curve redward at the faint end although it does show the redward turn at the bright end. The narrowness is due to the fact that all $\mathrm{O}$ and $\mathrm{B}$ stars have basically the same $V-R$ color and to the higher photometric accuracy in F702W and F555W compared to F336W. In the upper right of this plot one can also see the star Mk9, classified as an $\mathrm{M}$ supergiant by Melnick (1985). No red supergiants were found within R136 itself.

One other point of interest is that the color-magnitude diagrams of R136 and of the stars measured outside of 
TABLE 4. Photometry of stars outside R136 (PC6).

\begin{tabular}{|c|c|c|c|c|c|c|c|c|c|c|c|c|c|}
\hline ID & $\mathrm{x}$ & $\mathrm{y}$ & F555W & $\sigma$ & $336-555$ & $555-702$ & ID & $\mathrm{x}$ & $\mathrm{y}$ & F555W & $\sigma$ & $336-555$ & $555-702$ \\
\hline 6001 & 317.2 & 724.7 & 12.57 & 0.07 & -0.80 & 0.32 & 6041 & 314.7 & 651.4 & 15.57 & 0.05 & -0.99 & 0.17 \\
\hline 6002 & 199.8 & 568.3 & 12.67 & 0.05 & -1.08 & 0.15 & 6042 & 692.7 & 460.7 & 15.60 & 0.06 & -0.77 & 0.34 \\
\hline 6003 & 388.6 & 736.0 & 12.94 & 0.05 & -1.01 & 0.18 & 6043 & 179.8 & 699.8 & 15.62 & 0.06 & -0.96 & 0.24 \\
\hline 6004 & 314.5 & 232.1 & 12.95 & 0.01 & -0.73 & 0.32 & 6044 & 597.1 & 227.3 & 15.62 & 0.06 & -1.16 & 0.09 \\
\hline 6005 & 595.5 & 395.3 & 13.30 & 0.03 & -0.88 & 0.25 & 6045 & 543.9 & 395.6 & 15.68 & 0.06 & -0.97 & 0.14 \\
\hline 6006 & 542.3 & 269.1 & 13.36 & 0.03 & -1.03 & 0.16 & 6046 & 650.8 & 378.7 & 15.69 & 0.07 & -1.18 & 0.23 \\
\hline 6007 & 595.4 & 352.2 & 13.46 & 0.03 & -0.85 & 0.28 & 6047 & 250.7 & 459.4 & 15.70 & 0.04 & -0.65 & 0.45 \\
\hline 6008 & 76.7 & 327.7 & 13.52 & 0.05 & -0.87 & 0.20 & 6048 & 334.2 & 558.7 & 15.70 & 0.05 & -0.96 & 0.14 \\
\hline 6009 & 128.4 & 332.0 & 13.67 & 0.04 & -0.88 & 0.20 & 6049 & 153.4 & 558.8 & 15.79 & 0.06 & -0.81 & 0.21 \\
\hline 6010 & 582.4 & 243.0 & 13.81 & 0.04 & -1.18 & 0.09 & 6050 & 556.6 & 776.8 & 15.81 & 0.06 & -0.90 & 0.18 \\
\hline 6011 & 346.3 & 582.8 & 14.01 & 0.06 & -0.97 & 0.00 & 6051 & 130.0 & 310.5 & 15.82 & 0.08 & -0.75 & 0.20 \\
\hline 6012 & 264.0 & 621.4 & 14.08 & 0.06 & -1.14 & 0.12 & 6052 & 188.1 & 508.7 & 15.82 & 0.06 & -0.43 & 0.47 \\
\hline 6013 & 110.4 & 348.9 & 14.20 & 0.05 & -0.32 & -0.30 & 6053 & 479.0 & 282.5 & 15.90 & 0.04 & -0.96 & 0.17 \\
\hline 6014 & 87.5 & 304.2 & 14.21 & 0.05 & -0.87 & 0.17 & 6054 & 376.5 & 723.6 & 15.93 & 0.10 & -0.98 & 0.03 \\
\hline 6015 & 459.5 & 465.4 & 14.21 & 0.04 & -1.00 & 0.12 & 6055 & 461.9 & 381.6 & 15.93 & 0.05 & -1.07 & 0.16 \\
\hline 6016 & 568.1 & 434.0 & 14.39 & 0.04 & -0.83 & 0. & 6056 & 127.0 & 384.4 & 15.93 & 0.06 & -0.74 & 0.30 \\
\hline 6017 & 482.3 & 315.7 & 14.40 & 0.05 & -0.91 & 0.10 & 6057 & 483.3 & 447.3 & 15.97 & 0.04 & -0.99 & 0.09 \\
\hline 6018 & 608.3 & 417.4 & 14.47 & 0.04 & -0.70 & 0.32 & 6058 & 606.8 & 687.2 & 15.98 & 0.06 & -1.34 & 0.28 \\
\hline 6019 & 568.5 & 768.8 & 14.57 & 0.05 & -0.98 & 0.14 & 6059 & 425.8 & 53.1 & 15.99 & 0.05 & -1.04 & 0.15 \\
\hline 6020 & 75.5 & 66.5 & 14.66 & 0.05 & -1.02 & 0.14 & 6060 & 138.8 & 601.5 & 16.00 & 0.06 & -1.05 & 0.19 \\
\hline 6021 & 192.9 & 495.2 & 14.66 & 0.05 & -0.71 & 0.33 & 6061 & 387.0 & 581.4 & 16.03 & 0.06 & -0.96 & 0.19 \\
\hline 6022 & 273.3 & 696.2 & 14.69 & 0.05 & -1.08 & 0. & 6062 & 118.1 & 523.0 & 16.03 & 0.06 & -0.86 & 0.22 \\
\hline 6023 & 386.6 & 623.0 & 14.75 & 0.04 & -0.88 & 0.1 & 6063 & 455.8 & 622.0 & 16.05 & 0.05 & -0.92 & 0.27 \\
\hline 6024 & 242.0 & 767.1 & 14.99 & 0.07 & -1.05 & 0.06 & 6064 & 400.0 & 695.5 & 16.11 & 0.08 & -0.72 & 0.18 \\
\hline 6025 . & 294.4 & 686.9 & 14.99 & 0.07 & -0.89 & 0.08 & 6065 & 663.7 & 501.1 & 16.12 & 0.04 & -0.87 & 0.29 \\
\hline 6026 & 286.5 & 634.5 & 15.02 & 0.05 & -1.08 & 0.14 & 6066 & 410.4 & 713.8 & 16.15 & 0.07 & -0.62 & 0.06 \\
\hline 6027 & 739.8 & 775.3 & 15.14 & 0.07 & -0.89 & 0.23 & 6067 & 254.5 & 443.0 & 16.20 & 0.04 & -0.63 & 0.28 \\
\hline 6028 & 285.8 & 612.3 & 15.20 & 0.05 & -1.22 & 0.1 & 6068 & 478.1 & 551.8 & 16.20 & 0.05 & -0.87 & 0.18 \\
\hline 6029 & 596.2 & 711.7 & 15.25 & 0.06 & -0.86 & 0.14 & 6069 & 523.1 & 730.3 & 16.21 & 0.07 & -0.97 & 0.24 \\
\hline 6030 & 499.7 & 365.6 & 15.31 & 0.04 & -0.89 & 0.17 & 6070 & 435.4 & 641.0 & 16.24 & 0.05 & -0.04 & 0.83 \\
\hline 6031 & 332.9 & 566.0 & 15.34 & 0.05 & -1.08 & 0.19 & 6071 & 523.7 & 54.5 & 16.29 & 0.05 & -1.01 & 0.07 \\
\hline 6032 & 304.5 & 707.2 & 15.35 & 0.07 & -1.07 & 0.17 & 6072 & 245.0 & 671.4 & 16.29 & 0.07 & -1.09 & 0.21 \\
\hline 6033 & 710.3 & 386.4 & 15.37 & 0.04 & -0.86 & 0.26 & 6073 & 436.5 & 721.5 & 16.31 & 0.06 & -1.15 & 0.23 \\
\hline 6034 & 405.4 & 603.4 & 15.39 & 0.04 & -1.00 & 0.20 & 6074 & 280.6 & 264.6 & 16.32 & 0.03 & -0.53 & 0.19 \\
\hline 6035 & 254.4 & 698.0 & 15.44 & 0.05 & 1103 & 0.15 & 6075 & 670.2 & 138.4 & 16.32 & 0.05 & -1.11 & 0.06 \\
\hline 6036 & 576.0 & 702.1 & 15.45 & 0.06 & -0.98 & 0.23 & 6076 & 564.7 & 196.1 & 16.33 & 0.05 & -0.95 & 0.09 \\
\hline 6037 & 471.6 & 401.3 & 15.45 & 0.03 & -0.93 & 0.15 & 6077 & 575.8 & 337.7 & 16.35 & 0.06 & -0.56 & 0.24 \\
\hline 6038 & 599.8 & 375.1 & 15.49 & 0.05 & -0.36 & 0.14 & 6078 & 332.8 & 597.2 & 16.36 & 0.07 & -1.21 & 0.22 \\
\hline 6039 & 200.9 & 609.3 & 15.49 & 0.07 & - & -0.37 & 6079 & 704.2 & 400.8 & 16.37 & 0.06 & -0.74 & 0.30 \\
\hline 6040 & 625.9 & 478.6 & 15.50 & 0.07 & -0.68 & 0.33 & 6080 & 222.6 & 546.7 & 16.38 & 0.08 & -0.88 & 0.15 \\
\hline
\end{tabular}

(b)

\begin{tabular}{|c|c|c|c|c|c|c|c|c|c|c|c|c|c|}
\hline ID & $x$ & $y$ & F555W & $\sigma$ & $336-555$ & $555-702$ & ID & $x$ & $y$ & F555W & $\sigma$ & $336-555$ & $555-702$ \\
\hline 6081 & 596.9 & 371.5 & 16.38 & 0.10 & -1.09 & 0.27 & 6121 & 428.3 & 164.2 & 16.89 & 0.03 & -0.19 & 0.12 \\
\hline 6082 & 231.7 & 509.8 & 16.41 & 0.05 & -0.67 & 0.20 & 6122 & 244.9 & 592.5 & 16.90 & 0.07 & -1.00 & 0.14 \\
\hline 6083 & 392.8 & 333.6 & 16.42 & 0.04 & -0.73 & 0.19 & 6123 & 724.1 & 143.8 & 16.90 & 0.06 & -0.92 & 0.21 \\
\hline 6084 & 192.2 & 446.7 & 16.45 & 0.05 & -0.22 & 0.38 & 6124 & 644.9 & 300.8 & 16.91 & 0.06 & -0.89 & 0.15 \\
\hline 6085 & 554.9 & 386.1 & 16.45 & 0.06 & -0.77 & 0.13 & 6125 & 418.6 & 335.6 & 16.92 & 0.05 & -0.70 & 0.03 \\
\hline 6086 & 668.3 & 676.1 & 16.46 & 0.04 & -0.95 & 0.21 & 6126 & 451.4 & 424.9 & 16.92 & 0.06 & -0.73 & 0.18 \\
\hline 6087 & 143.1 & 155.8 & 16.48 & 0.05 & -0.91 & 0.17 & 6127 & 541.7 & 294.5 & 16.92 & 0.07 & -0.46 & 0.23 \\
\hline 6088 & 434.5 & 617.7 & 16.48 & 0.05 & -0.98 & 0.18 & 6128 & 456.8 & 625.4 & 16.94 & 0.10 & -0.70 & .0 .03 \\
\hline 6089 & 487.8 & 307.7 & 16.49 & 0.12 & -1.18 & 0.42 & 6129 & 456.5 & 645.9 & 16.94 & 0.06 & -0.86 & 0.12 \\
\hline 6090 & 304.2 & 584.5 & 16.50 & 0.06 & -0.89 & 0.15 & 6130 & 47.2 & 244.9 & 16.95 & 0.08 & -0.97 & 0.04 \\
\hline 6091 & 611.4 & 553.5 & 16.51 & 0.05 & -0.84 & 0.27 & 6131 & 221.2 & 469.5 & 16.96 & 0.06 & -0.50 & 0.16 \\
\hline 6092 & 728.5 & 205.6 & 16.52 & 0.05 & -1.02 & 0.11 & 6132 & 357.4 & 556.2 & 16.97 & 0.08 & -0.90 & 0.11 \\
\hline 6093 & 240.0 & 612.0 & 16.53 & 0.07 & -1.03 & 0.08 & 6133 & 693.0 & 196.8 & 16.97 & 0.07 & -1.06 & 0.16 \\
\hline 6094 & 613.2 & 428.7 & 16.53 & 0.07 & -0.20 & 0.14 & 6134 & 182.5 & 611.7 & 16.97 & 0.09 & -0.73 & 0.09 \\
\hline 6095 & 250.0 & 464.3 & 16.53 & 0.07 & -0.76 & 0.13 & 6135 & 613.7 & 177.8 & 16.97 & 0.06 & -1.02 & 0.18 \\
\hline 6096 & 485.7 & 573.7 & 16.54 & 0.06 & -0.94 & 0.27 & 6136 & 439.4 & 540.2 & 16.98 & 0.05 & -0.89 & 0.18 \\
\hline 6097 & 561.7 & 393.2 & 16.57 & 0.06 & -0.45 & 0.21 & 6137 & 489.0 & 214.8 & 16.98 & 0.05 & -0.70 & 0.17 \\
\hline 6098 & 174.8 & 706.1 & 16.61 & 0.06 & -1.08 & 0.22 & 6138 & 272.5 & 316.6 & 16.99 & 0.04 & -0.69 & 0.27 \\
\hline 6099 & 515.4 & 346.3 & 16.64 & 0.04 & -0.60 & 0.18 & 6139 & 539.2 & 306.1 & 16.99 & 0.06 & -0.74 & 0.27 \\
\hline 6100 & 490.7 & 633.8 & 16.64 & 0.08 & -0.84 & 0.29 & 6140 & 453.0 & 754.8 & 17.01 & 0.06 & -0.89 & 0.12 \\
\hline 6101 & 436.4 & 475.5 & 16.65 & 0.06 & -0.93 & 0.14 & 6141 & 179.2 & 671.9 & 17.04 & 0.06 & -0.60 & 0.17 \\
\hline 6102 & 527.1 & 514.5 & 16.66 & 0.07 & 1.92 & 0.12 & 6142 & 365.8 & 579.3 & 17.05 & 0.09 & -0.78 & 0.12 \\
\hline 6103 & 483.3 & 566.0 & 16.67 & 0.07 & -0.69 & 0.04 & 6143 & 483.8 & 308.4 & 17.05 & 0.11 & -0.47 & 0.11 \\
\hline 6104 & 491.9 & 391.8 & 16.68 & 0.05 & -0.42 & 0.12 & 6144 & 318.2 & 604.6 & 17.06 & 0.07 & -0.63 & 0.21 \\
\hline 6105 & 526.9 & 399.4 & 16.69 & 0.06 & -0.74 & 0.11 & 6145 & 363.2 & 572.1 & 17.06 & 0.11 & -0.72 & 0.09 \\
\hline 6106 & 286.3 & 707.5 & 16.69 & 0.10 & -0.90 & 0.31 & 6146 & 587.8 & 330.0 & 17.06 & 0.10 & - & 1.11 \\
\hline 6107 & 528.2 & 572.8 & 16.75 & 0.07 & -1.16 & 0.16 & 6147 & 576.2 & 357.7 & 17.07 & 0.12 & -0.66 & 0.32 \\
\hline 6108 & 438.2 & 444.8 & 16.75 & 0.06 & -0.80 & 0.15 & 6148 & 422.1 & 294.4 & 17.07 & 0.05 & -0.76 & 0.21 \\
\hline 6109 & 195.4 & 641.0 & 16.77 & 0.05 & -0.87 & 0.16 & 6149 & 433.5 & 763.4 & 17.09 & 0.07 & -0.91 & 0.23 \\
\hline 6110 & 373.8 & 617.6 & 16.80 & 0.07 & -0.69 & 0.19 & 6150 & 607.8 & 364.6 & 17.09 & 0.13 & - & 0.41 \\
\hline 6111 & 194.2 & 751.4 & 16.80 & 0.07 & -0.68 & 0.34 & 6151 & 290.6 & 95.8 & 17.10 & 0.04 & -0.51 & 0.07 \\
\hline 6112 & 322.6 & 591.2 & 16.84 & 0.07 & -1.16 & 0.24 & 6152 & 784.0 & 90.7 & 17.12 & 0.06 & -0.93 & 0.19 \\
\hline 6113 & 699.0 & 488.2 & 16.84 & 0.07 & -0.65 & 0.43 & 6153 & 63.5 & 320.2 & 17.13 & 0.12 & -0.68 & 0.27 \\
\hline 6114 & 162.6 & 662.6 & 16.84 & 0.06 & -0.73 & 0.23 & 6154 & 493.7 & 436.6 & 17.13 & 0.06 & -0.60 & 0.17 \\
\hline 6115 & 328.0 & 579.7 & 16.84 & 0.08 & -1.04 & 0.12 & 6155 & 603.5 & 641.3 & 17.14 & 0.05 & -0.47 & 0.57 \\
\hline 6116 & 339.5 & 78.5 & 16.85 & 0.03 & - & 0.16 & 6156 & 307.0 & 557.5 & 17.16 & 0.10 & -0.90 & 0.18 \\
\hline 6117 & 366.1 & 576.6 & 16.87 & 0.09 & -1.08 & 0.16 & 6157 & 187.4 & 529.8 & 17.16 & 0.12 & -0.80 & 0.21 \\
\hline 6118 & 242.7 & 578.6 & 16.88 & 0.06 & -0.56 & 0.13 & 6158 & 437.5 & 500.3 & 17.19 & 0.07 & -0.88 & 0.12 \\
\hline 6119 & 206.4 & 508.5 & 16.88 & 0.07 & -0.85 & 0.17 & 6159 & 247.0 & 344.6 & 17.19 & 0.06 & -0.27 & 0.22 \\
\hline 6120 & 553.8 & 400.1 & 16.89 & 0.07 & -0.52 & 0.13 & 6160 & 304.6 & 599.4 & 17.21 & 0.08 & - & 0.18 \\
\hline
\end{tabular}


TABLE 4. (continued)

\begin{tabular}{|c|c|c|c|c|c|c|c|c|c|c|c|c|c|}
\hline ID & $x$ & $\mathrm{y}$ & F555W & $\sigma$ & $336-555$ & $555-702$ & ID & $\mathrm{x}$ & $\mathrm{y}$ & F555W & $\sigma$ & 555 & \\
\hline 6161 & 486.6 & 309.1 & 17.22 & 0.17 & -0.62 & -0.05 & 6201 & 306.5 & 311.0 & 17.55 & 0.06 & -0.27 & 0.22 \\
\hline 6162 & 660.0 & 302.1 & 17.22 & 0.06 & -0.55 & 0.22 & 6202 & 387.5 & 666.2 & 17.56 & 0.07 & -0.63 & 0.37 \\
\hline 6163 & 364.8 & 552.8 & 17.23 & 0.08 & -0.67 & 0.23 & 6203 & 564.9 & 399.2 & 17.56 & 0.13 & -0.77 & 0.36 \\
\hline 6164 & 145.7 & 691.5 & 17.24 & 0.07 & -0.71 & 0.24 & 6204 & 475.0 & 774.2 & 17.57 & 0.08 & -0.74 & 0.20 \\
\hline 6165 & 415.7 & 207.4 & 17.24 & 0.04 & -0.40 & 0.09 & 6205 & 218.8 & 638.0 & 17.58 & 0.06 & -0.51 & 0.18 \\
\hline 6166 & 651.1 & 381.4 & 17.25 & 0.21 & - & -0.62 & 6206 & 711.5 & 775.3 & 17.60 & 0.08 & -1.04 & 0.26 \\
\hline 6167 & 529.8 & 756.3 & 17.26 & 0.10 & -0.92 & 0.32 & 6207 & 310.4 & 549.8 & 17.61 & 0.11 & -0.61 & 0.26 \\
\hline 6168 & 442.9 & 431.4 & 17.26 & 0.08 & -0.61 & 0.10 & 6208 & 616.8 & 796.8 & 17.62 & 0.09 & -0.68 & 0.12 \\
\hline 6169 & 97.1 & 79.7 & 17.28 & 0.09 & -0.79 & 0.14 & 6209 & 485.2 & 462.8 & 17.62 & 0.11 & -1.08 & 0.25 \\
\hline 6170 & 198.6 & 414.0 & 17.29 & 0.05 & -0.60 & 0.23 & 6210 & 526.0 & 620.5 & 17.62 & 0.07 & -0.88 & 0.20 \\
\hline 6171 & 256.1 & 721.2 & 17.30 & 0.09 & -0.73 & 0.20 & 6211 & 117.9 & 689.5 & 17.64 & 0.08 & -0.43 & 0.25 \\
\hline 6172 & 322.6 & 345.1 & 17.31 & 0.06 & -0.81 & 0.22 & 6212 & 239.9 & 761.3 & 17.64 & 0.19 & - & -0.20 \\
\hline 6173 & 522.9 & 243.6 & 17.33 & 0.09 & -0.69 & 0.17 & 6213 & 448.4 & 501.5 & 17.65 & 0.09 & -0.55 & 0.09 \\
\hline 6174 & 240.4 & 433.2 & 17.34 & 0.09 & -1.18 & 0.37 & 6214 & 411.5 & 642.1 & 17.66 & 0.08 & -0.14 & 0.24 \\
\hline 6175 & 520.2 & 259.5 & 17.34 & 0.09 & -0.99 & 0.26 & 6215 & 368.4 & 623.2 & 17.66 & 0.10 & - & 0.20 \\
\hline 6176 & 527.9 & 317.6 & 17.35 & 0.06 & -0.18 & 0.12 & 6216 & 308.1 & 623.5 & 17.67 & 0.13 & - & 0.19 \\
\hline 6177 & 178.1 & 48.3 & 17.35 & 0.05 & -0.83 & 0.12 & 6217 & 45.8 & 319.2 & 17.67 & 0.15 & - & 0.25 \\
\hline 6178 & 562.3 & 777.8 & 17.36 & 0.14 & -1.26 & 0.22 & 6218 & 200.3 & 590.6 & 17.67 & 0.31 & - & -0.04 \\
\hline 6179 & 427.5 & 453.7 & 17.36 & 0.11 & -0.82 & 0.27 & 6219 & 476.4 & 413.2 & 17.68 & 0.08 & .0 .10 & 0.09 \\
\hline 6180 & 663.2 & 765.5 & 17.36 & 0.08 & -0.66 & 0.38 & 6220 & 557.7 & 763.9 & 17.69 & 0.15 & -0.32 & 0.35 \\
\hline 6181 & 104.2 & 288.3 & 17.38 & 0.18 & -0.09 & 0.26 & 6221 & 222.8 & 616.8 & 17.70 & 0.10 & -0.89 & 0.00 \\
\hline 6182 & 430.2 & 405.2 & 17.38 & 0.07 & -0.85 & 0.22 & 6222 & 310.2 & 342.7 & 17.70 & 0.08 & -0.74 & 0.23 \\
\hline 6183 & 317.0 & 557.0 & 17.40 & 0.08 & -0.95 & -0.05 & 6223 & 235.1 & 533.5 & 17.73 & 0.10 & -0.57 & 0.30 \\
\hline 6184 & 681.5 & 298.5 & 17.41 & 0.06 & -0.72 & 0.17 & 6224 & 257.2 & 408.2 & 17.73 & 0.12 & -1.08 & 0.29 \\
\hline 6185 & 473.6 & 277.6 & 17.42 & 0.09 & -0.41 & 0.16 & 6225 & 325.4 & 268.1 & 17.74 & 0.11 & - & 0.52 \\
\hline 6186 & 349.3 & 606.7 & 17.42 & 0.12 & - & 0.20 & 6226 & 226.3 & 497.4 & 17.74 & 0.12 & -0.39 & 0.28 \\
\hline 6187 & 588.3 & 444.5 & 17.44 & 0.10 & - & 0.18 & 6227 & 616.5 & 774.0 & 17.74 & 0.10 & -0.46 & 0.18 \\
\hline 6188 & 487.7 & 444.1 & 17.44 & 0.07 & -0.59 & 0.24 & 6228 & 474.4 & 604.1 & 17.75 & 0.08 & -0.38 & 0.21 \\
\hline 6189 & 686.7 & 618.9 & 17.46 & 0.07 & -0.44 & 0.32 & 6229 & 188.8 & 676.7 & 17.75 & 0.11 & -0.27 & 0.29 \\
\hline 6190 & 414.2 & 613.5 & 17.46 & 0.10 & - & 0.31 & 6230 & 588.2 & 193.1 & 17.76 & 0.08 & -0.86 & 0.09 \\
\hline 6191 & 434.8 & 279.5 & 17.47 & 0.06 & -0.67 & 0.40 & 6231 & 313.9 & 685.8 & 17.76 & 0.19 & -0.56 & 0.27 \\
\hline 6192 & 211.4 & 425.9 & 17.47 & 0.06 & - & 0.32 & 6232 & 593.0 & 649.0 & 17.77 & 0.07 & -0.96 & 0.29 \\
\hline 6193 & 302.4 & 566.5 & 17.48 & 0.09 & -0.62 & 0.25 & 6233 & 546.7 & 47.1 & 17.77 & 0.08 & -1.15 & 0.28 \\
\hline 6194 & 31.1 & 510.4 & 17.49 & 0.07 & -1.01 & 0.20 & 6234 & 50.3 & 215.8 & 17.78 & 0.07 & -0.58 & 0.07 \\
\hline 6195 & 238.1 & 687.9 & 17.50 & 0.09 & -0.95 & 0.26 & 6235 & 167.1 & 614.8 & 17.79 & 0.10 & - & 0.15 \\
\hline 6196 & 514.3 & 417.4 & 17.52 & 0.06 & -0.61 & 0.23 & 6236 & 346.5 & 565.8 & 17.80 & 0.12 & -0.08 & 0.17 \\
\hline 6197 & 586.8 & 592.2 & 17.52 & 0.06 & -0.99 & 0.14 & 6237 & 493.1 & 443.5 & 17.80 & 0.08 & -0.31 & 0.34 \\
\hline 6198 & 157.6 & 63.3 & 17.52 & 0.05 & -0.80 & 0.16 & 6238 & 237.1 & 665.6 & 17.80 & 0.10 & -1.06 & 0.38 \\
\hline 6199 & 253.0 & 625.6 & 17.53 & 0.13 & -1.03 & 0.28 & 6239 & 530.7 & 301.2 & 17.80 & 0.13 & -0.57 & 0.07 \\
\hline 6200 & 443.2 & 311.7 & 17.54 & 0.07 & -0.62 & 0.17 & 6240 & 556.4 & 436.4 & 17.81 & 0.13 & -0.77 & -0.05 \\
\hline
\end{tabular}

(d)

\begin{tabular}{|c|c|c|c|c|c|c|c|c|c|c|c|c|c|}
\hline ID & $x$ & $\mathrm{y}$ & F555W & $\sigma$ & $336-555$ & $555-702$ & ID & $\mathrm{x}$ & $\mathrm{y}$ & F555W & $\sigma$ & $336-555$ & $555-702$ \\
\hline 6241 & 363.9 & 570.2 & 17.82 & 0.19 & -0.83 & 0.30 & 6281 & 474.0 & 324.8 & 18.05 & 0.14 & -0.85 & 0.52 \\
\hline 6242 & 430.4 & 781.3 & 17.82 & 0.09 & - & -0.01 & 6282 & 314.4 & 330.7 & 18.06 & 0.09 & - & 0.06 \\
\hline 6243 & 296.0 & 588.5 & 17.83 & 0.14 & - & 0.00 & 6283 & 140.9 & 639.1 & 18.07 & 0.09 & -0.32 & 0.14 \\
\hline 6244 & 163.6 & 495.9 & 17.83 & 0.09 & - & 0.64 & 6284 & 513.6 & 341.0 & 18.07 & 0.12 & - & 0.43 \\
\hline 6245 & 430.4 & 566.3 & 17.83 & 0.07 & -0.15 & 0.16 & 6285 & 350.9 & 163.2 & 18.07 & 0.08 & -0.87 & 0.17 \\
\hline 6246 & 650.0 & 514.0 & 17.86 & 0.08 & -0.89 & 0.24 & 6286 & 290.3 & 139.3 & 18.08 & 0.07 & -0.67 & 0.15 \\
\hline 6247 & 221.9 & 492.0 & 17.88 & 0.14 & - & 0.38 & 6287 & 114.4 & 604.7 & 18.09 & 0.12 & - & 0.19 \\
\hline 6248 & 258.1 & 456.1 & 17.88 & 0.14 & - & -0.05 & 6288 & 209.1 & 548.3 & 18.10 & 0.40 & -1.46 & 0.25 \\
\hline 249 & 350.4 & 339.4 & 17.88 & 0.16 & - & 0.27 & 6289 & 196.9 & 654.0 & 18.10 & 0.10 & -0.55 & 0.12 \\
\hline 250 & 329.8 & 192.3 & 17.89 & 0.10 & -1.05 & 0.43 & 6290 & 440.5 & 487.5 & 18.11 & 0.15 & - & 0.28 \\
\hline 5251 & 649.0 & 126.2 & 17.90 & 0.08 & -0.77 & 0.17 & 6291 & 483.6 & 439.4 & 18.11 & 0.10 & -0.35 & 0.37 \\
\hline 6252 & 284.5 & 339.5 & 17.90 & 0.08 & - & 0.26 & 6292 & 232.7 & 600.6 & 18.11 & 0.12 & - & 0.19 \\
\hline 6253 & 180.6 & 204.9 & 17.90 & 0.05 & - & 0.23 & 6293 & 206.9 & 405.1 & 18.12 & 0.09 & - & 0.24 \\
\hline 6254 & 429.2 & 626.2 & 17.90 & 0.10 & -0.53 & 0.12 & 6294 & 153.4 & 624.4 & 18.12 & 0.09 & -0.70 & 0.16 \\
\hline 255 & 434.3 & 694.8 & 17.91 & 0.08 & -1.07 & 0.15 & 6295 & 367.4 & 258.4 & 18.12 & 0.07 & - & 0.35 \\
\hline 6256 & 721.3 & 448.8 & 17.91 & 0.08 & -0.56 & 0.33 & 6296 & 669.5 & 505.7 & 18.12 & 0. & -0.95 & 0.24 \\
\hline 6257 & 496.5 & 547.4 & 17.92 & 0.10 & -0.91 & 0.23 & 6297 & 55.9 & 653.8 & 18.13 & 0.13 & -1.09 & 0.38 \\
\hline 6258 & 284.8 & 330.9 & 17.93 & 0.08 & - & 0.19 & 6298 & 393.0 & 567.2 & 18.13 & 0.18 & -0.21 & 0.10 \\
\hline 6259 & 153.4 & 752.6 & 17.94 & 0.08 & -0.29 & 0.46 & 6299 & 124.8 & 730.8 & 18.13 & 0.09 & - & .80 \\
\hline 6260 & 430.9 & 289.1 & 17.94 & 0.08 & -0.29 & 0.20 & 6300 & 305.9 & 560.0 & 18.13 & 0.19 & - & 0.00 \\
\hline 6261 & 310.7 & 283.0 & 17.95 & 0.08 & - & 0.30 & 6301 & 259.2 & 737.6 & 18.14 & 0.1 & -0.72 & 0.20 \\
\hline 6262 & 141.9 & 285.3 & 17.95 & 0.09 & - & 0.17 & 6302 & 324.4 & 582.6 & 18.14 & 0.19 & -0.60 & 0.11 \\
\hline 6263 & 131.5 & 647.9 & 17.95 & 0.09 & -0.28 & 0.21 & 6303 & 493.1 & 633.3 & 18.14 & 0.23 & - & -0.50 \\
\hline 6264 & 490.3 & 307.0 & 17.96 & 0.25 & - & -0.35 & 6304 & 677.5 & 645.5 & 18.15 & 0.07 & -0.84 & 0.24 \\
\hline 6265 & 442.5 & 631.9 & 17.96 & 0.10 & -0.39 & 0.32 & 6305 & 356.0 & 571.8 & 18.15 & 0.18 & - & 0.40 \\
\hline 6266 & 544.1 & 221.2 & 17.96 & 0.10 & 0.10 & 0.16 & 6306 & 195.2 & 405.2 & 18.15 & 0.09 & - & 0.46 \\
\hline 6267 & 317.5 & 310.9 & 17.97 & 0.08 & -0.95 & 0.20 & 6307 & 361.6 & 279.8 & 18.15 & 0.07 & -0.68 & 0.23 \\
\hline 6268 & 450.9 & 632.9 & 17.97 & 0.10 & -0.55 & 0.10 & 6308 & 235.5 & 570.3 & 18.15 & 0.14 & -0.99 & 0.00 \\
\hline 6269 & 461.9 & 242.2 & 17.97 & 0.07 & - & 0.18 & 6309 & 55.1 & 302.8 & 18.18 & 0.22 & - & 0.53 \\
\hline 6270 & 654.4 & 726.2 & 17.99 & 0.07 & -0.79 & 0.33 & 6310 & 318.5 & 574.0 & 18.19 & 0.14 & - & 0.26 \\
\hline 6271 & 84.9 & 391.5 & 17.99 & 0.07 & -0.64 & 0.72 & 6311 & 466.4 & 290.4 & 18.19 & 0.18 & - & 0.21 \\
\hline 6272 & 564.8 & 97.5 & 17.99 & 0.0 & -0.91 & 0 & 6312 & 425.4 & 564.8 & 18.20 & 0.1 & -0.65 & 0.04 \\
\hline 6273 & 555.0 & 363.5 & 18.00 & 0.1 & - & 0.33 & 6313 & 306.3 & 669.2 & 18.20 & 0.20 & - & 0.49 \\
\hline 6274 & 225.9 & 314.3 & 18.00 & 0.07 & - & 0.12 & 6314 & 391.1 & 673.7 & 18.20 & 0.12 & - & 0.25 \\
\hline 6275 & 287.0 & 664.9 & 18.01 & 0.17 & - & 0.33 & 6315 & 252.7 & 123.4 & 18.25 & 0.08 & - & 0.15 \\
\hline 6276 & 35.9 & 704.2 & 18.01 & 0.07 & -1.00 & 0.40 & 6316 & 509.1 & 388.6 & 18.25 & 0.14 & - & 0.13 \\
\hline 6277 & 225.9 & 614.8 & 18.01 & 0.13 & - & 0.27 & 6317 & 289.2 & 562.0 & 18.25 & 0.30 & - & 0.52 \\
\hline 6278 & 453.3 & 417.1 & 18.02 & 0.09 & -0.45 & 0.33 & 6318 & 381.6 & 604.8 & 18.26 & 0.19 & - & 0.03 \\
\hline 6279 & 209.1 & 339.2 & 18.04 & 0.07 & - & 0.50 & 6319 & 538.6 & 483.1 & 18.26 & 0.08 & - & 0.32 \\
\hline 6280 & 593.0 & 676.9 & 18.05 & 0.15 & -0.82 & 0.10 & 6320 & 390.8 & 344.1 & 18.26 & 0.15 & -1.06 & 0.10 \\
\hline
\end{tabular}


TABLE 4. (continued)

\begin{tabular}{|c|c|c|c|c|c|c|c|c|c|c|c|c|c|}
\hline ID & $x$ & $y$ & F555W & $\sigma$ & $336-555$ & $555-702$ & ID & $x$ & $\mathrm{y}$ & F555W & $\sigma$ & $336-555$ & $555-702$ \\
\hline 6321 & 782.0 & 477.0 & 18.26 & 0.08 & -0.68 & 0.58 & 6361 & 793.7 & 376.5 & 18.57 & 0.15 & - & 0.35 \\
\hline 6322 & 132.7 & 163.1 & 18.27 & 0.09 & - & 0.19 & 6362 & 604.6 & 551.6 & 18.57 & 0.14 & - & 0.55 \\
\hline 6323 & 426.2 & 393.1 & 18.27 & 0.12 & - & 0.16 & 6363 & 662.5 & 576.4 & 18.58 & 0.10 & - & 0.40 \\
\hline 6324 & 681.7 & 397.0 & 18.29 & 0.15 & - & 0.09 & 6364 & 433.4 & 372.5 & 18.58 & 0.12 & - & 0.22 \\
\hline 6325 & 328.2 & 573.9 & 18.30 & 0.19 & -0.70 & 0.14 & 6365 & 484.5 & 603.6 & 18.59 & 0.13 & - & 0.40 \\
\hline 6326 & 198.6 & 462.8 & 18.30 & 0.14 & -0.58 & 0.32 & 6366 & 573.0 & 292.7 & 18.59 & 0.22 & -1.29 & 0.03 \\
\hline 6327 & 507.7 & 363.8 & 18.30 & 0.15 & - & 0.44 & 6367 & 295.2 & 333.9 & 18.60 & 0.13 & - & 0.26 \\
\hline 6328 & 635.9 & 441.1 & 18.31 & 0.19 & - & 0.31 & 6368 & 322.6 & 64.9 & 18.62 & 0.10 & -1.36 & 0.24 \\
\hline 6329 & 433.1 & 533.7 & 18.31 & 0.11 & -0.93 & 0.28 & 6369 & 389.1 & 125.7 & 18.64 & 0.11 & - & 0.13 \\
\hline 6330 & 678.8 & 686.6 & 18.31 & 0.09 & -1.15 & 0.25 & 6370 & 481.6 & 409.6 & 18.65 & 0.15 & - & -0.22 \\
\hline 6331 & 315.0 & 334.7 & 18.32 & 0.12 & -0.49 & 0.09 & 6371 & 604.6 & 633.2 & 18.65 & 0.10 & - & 0.89 \\
\hline 6332 & 552.7 & 444.2 & 18.34 & 0.17 & - & 0.47 & 6372 & 375.5 & 610.5 & 18.65 & 0.23 & - & 0.30 \\
\hline 6333 & 618.3 & 495.6 & 18.34 & 0.13 & - & 0.57 & 6373 & 450.1 & 436.0 & 18.66 & 0.20 & - & 0.29 \\
\hline 6334 & 744.6 & 271.1 & 18.35 & 0.07 & -0.78 & 0.17 & 6374 & 579.0 & 480.5 & 18.68 & 0.09 & - & 0.39 \\
\hline 6335 & 607.6 & 320.0 & 18.40 & 0.24 & - & 0.38 & 6375 & 514.9 & 254.3 & 18.69 & 0.24 & - & 0.50 \\
\hline 6336 & 194.3 & 483.7 & 18.40 & 0.22 & - & 0.12 & 6376 & 489.1 & 745.4 & 18.70 & 0.15 & - & 0.06 \\
\hline 6337 & 535.3 & 319.6 & 18.41 & 0.12 & - & -0.56 & 6377 & 447.4 & 717.9 & 18.70 & 0.16 & - & 0.11 \\
\hline 6338 & 492.6 & 547.6 & 18.42 & 0.14 & - & 0.34 & 6378 & 256.7 & 252.8 & 18.71 & 0.10 & - & 0.44 \\
\hline 6339 & 200.3 & 651.3 & 18.43 & 0.12 & - & 0.06 & 6379 & 120.8 & 684.8 & 18.71 & 0.14 & - & 0.17 \\
\hline 6340 & 509.0 & 269.3 & 18.44 & 0.16 & - & 0.35 & 6380 & 730.4 & 198.0 & 18.71 & 0.14 & -0.92 & 0.61 \\
\hline 6341 & 302.6 & 608.3 & 18.45 & 0.21 & -0.89 & 0.30 & 6381 & 451.1 & 461.4 & 18.72 & 0.32 & - & 0.51 \\
\hline 6342 & 729.8 & 231.0 & 18.46 & 0.11 & -1.18 & 0.30 & 6382 & 323.3 & 578.0 & 18.72 & 0.27 & - & 0.43 \\
\hline 6343 & 241.0 & 604.5 & 18.46 & 0.15 & - & 0.00 & 6383 & 448.5 & 711.8 & 18.72 & 0.14 & - & 0.30 \\
\hline 6344 & 451.9 & 362.6 & 18.47 & 0.11 & -0.90 & 0.49 & 6384 & 162.9 & 528.7 & 18.72 & 0.23 & -0.77 & 0.55 \\
\hline 6345 & 427.1 & 260.0 & 18.47 & 0.10 & - & 0.18 & 6385 & 280.8 & 301.5 & 18.74 & 0.12 & -0.81 & 0.48 \\
\hline 6346 . & 490.3 & 377.9 & 18.48 & 0.17 & - & 0.33 & 6386 & 415.4 & 589.5 & 18.75 & 0.20 & - & 0.17 \\
\hline 6347 & 496.6 & 285.2 & 18.48 & 0.20 & - & 0.02 & 6387 & 460.1 & 610.4 & 18.76 & 0.17 & - & 0.40 \\
\hline 6348 & 498.4 & 551.2 & 18.48 & 0.14 & - & 0.04 & 6388 & 444.8 & 604.3 & 18.76 & 0.17 & - & 0.21 \\
\hline 6349 & 499.3 & 532.0 & 18.48 & 0.13 & - & 0.23 & 6389 & 236.4 & 163.8 & 18.77 & 0.12 & - & 0.40 \\
\hline 6350 & 512.5 & 478.2 & 18.50 & 0.08 & - & 0.34 & 6390 & 310.7 & 274.6 & 18.80 & 0.15 & -1.00 & 0.62 \\
\hline 6351 & 461.4 & 374.6 & 18.50 & 0.12 & - & -0.14 & 6391 & 573.1 & 288.5 & 18.81 & 0.27 & - & 0.46 \\
\hline 6352 & 212.2 & 403.2 & 18.51 & 0.10 & - & 0.38 & 6392 & 436.8 & 457.9 & 18.81 & 0.32 & - & 0.29 \\
\hline 6353 & 304.2 & 326.6 & 18.51 & 0.12 & -0.54 & 0.16 & 6393 & 199.3 & 336.5 & 18.82 & 0.13 & - & 0.68 \\
\hline 6354 & 592.0 & 477.9 & 18.51 & 0.08 & - & 0.28 & 6394 & 458.1 & 664.9 & 18.82 & 0.19 & - & 0.16 \\
\hline 6355 & 575.9 & 519.4 & 18.52 & 0.10 & - & 0.40 & 6395 & 291.8 & 166.7 & 18.83 & 0.11 & - & 0.15 \\
\hline 6356 & 578.2 & 524.5 & 18.52 & 0.10 & - & 0.00 & 6396 & 83.1 & 459.6 & 18.83 & 0.11 & -0.88 & -0.02 \\
\hline 6357 & 783.0 & 328.1 & 18.52 & 0.09 & - & 0.20 & 6397 & 213.4 & 719.7 & 18.83 & 0.13 & - & 0.51 \\
\hline 6358 & 469.2 & 503.9 & 18.53 & 0.14 & -0.74 & 0.23 & 6398 & 268.5 & 343.1 & 18.84 & 0.18 & - & -0.04 \\
\hline 6359 & 469.0 & 432.0 & 18.56 & 0.15 & - & 0.13 & 6399 & 558.2 & 329.6 & 18.84 & 0.19 & - & 0.13 \\
\hline 6360 & 290.7 & 564.2 & 18.56 & 0.28 & - & -0.02 & 6400 & 135.5 & 389.6 & 18.86 & 0.20 & - & 0.43 \\
\hline
\end{tabular}

(f)

\begin{tabular}{|c|c|c|c|c|c|c|c|c|c|c|c|c|c|}
\hline ID & $\mathrm{x}$ & y & F555W & $\sigma$ & $336-555$ & $555-702$ & ID & $x$ & $\mathrm{y}$ & F555W & $\sigma$ & $336-555$ & $555-702$ \\
\hline 6401 & 232.9 & 332.3 & 18.89 & 0.14 & - & 0.15 & 6440 & 503.8 & 314.5 & 19.26 & 0.33 & - & 0.33 \\
\hline 6402 & 546.3 & 547.2 & 18.90 & 0.14 & - & 0.10 & 6441 & 438.6 & 367.7 & 19.27 & 0.17 & - & 0.49 \\
\hline 6403 & 493.0 & 417.8 & 18.91 & 0.21 & - & 0.04 & 6442 & 688.2 & 730.8 & 19.27 & 0.16 & -0.98 & 0.33 \\
\hline 6404 & 45.8 & 719.2 & 18.92 & 0.13 & -1.62 & 0.58 & 6443 & 420.8 & 241.3 & 19.29 & 0.17 & - & 0.20 \\
\hline 6405 & 370.1 & 109.5 & 18.95 & 0.11 & - & 0.34 & 6444 & 441.9 & 379.6 & 19.29 & 0.21 & - & 0.25 \\
\hline 6406 & 284.1 & 295.3 & 18.96 & 0.12 & - & 0.66 & 6445 & 87.0 & 516.5 & 19.33 & 0.16 & - & 0.65 \\
\hline 6407 & 193.6 & 656.1 & 18.96 & 0.20 & - & 0.25 & 6446 & 451.8 & 357.4 & 19.33 & 0.24 & - & 0.06 \\
\hline 6408 & 205.2 & 381.0 & 18.96 & 0.14 & - & 0.49 & 6447 & 124.8 & 632.2 & 19.34 & 0.20 & - & 0.71 \\
\hline 6409 & 475.6 & 410.1 & 18.96 & 0.24 & -1.34 & 0.53 & 6448 & 343.5 & 169.9 & 19.34 & 0.19 & - & 0.35 \\
\hline 6410 & 476.8 & 138.9 & 18.97 & 0.12 & - & 0.05 & 6449 & 419.8 & 342.8 & 19.35 & 0.25 & - & 0.30 \\
\hline 6411 & 489.1 & 532.1 & 18.97 & 0.17 & - & 0.14 & 6450 & 480.3 & 589.2 & 19.37 & 0.26 & - & 0.53 \\
\hline 6412 & 423.2 & 264.7 & 18.98 & 0.18 & - & 0.23 & 6451 & 467.8 & 306.3 & 19.37 & 0.37 & - & -0.13 \\
\hline 6413 & 590.6 & 693.6 & 18.98 & 0.34 & - & 0.45 & 6452 & 562.8 & 603.3 & 19.40 & 0.20 & - & 0.08 \\
\hline 6414 & 33.2 & 689.7 & 18.98 & 0.14 & - & 0.35 & 6453 & 429.9 & 365.6 & 19.40 & 0.23 & - & 0.21 \\
\hline 6415 & 652.7 & 565.6 & 19.00 & 0.12 & - & 0.22 & 6454 & 113.8 & 680.9 & 19.41 & 0.25 & - & 0.43 \\
\hline 6416 & 430.0 & 612.1 & 19.00 & 0.23 & - & 0.64 & 6455 & 168.5 & 736.0 & 19.41 & 0.23 & - & 0.54 \\
\hline 6417 & 235.3 & 623.9 & 19.01 & 0.22 & - & 0.31 & 6456 & 433.6 & 343.5 & 19.46 & 0.28 & - & 0.02 \\
\hline 6418 & 499.8 & 302.0 & 19.01 & 0.24 & - & -0.03 & 6457 & 371.5 & 312.2 & 19.47 & 0.23 & - & 0.41 \\
\hline 6419 & 531.2 & 571.4 & 19.02 & 0.34 & - & 0.17 & 6458 & 232.0 & 286.6 & 19.48 & 0.19 & - & 0.75 \\
\hline 6420 & 581.9 & 558.9 & 19.03 & 0.18 & - & 0.42 & 6459 & 432.1 & 352.4 & 19.51 & 0.26 & - & -0.18 \\
\hline 6421 & 287.0 & 151.3 & 19.03 & 0.12 & - & -0.08 & 6460 & 517.8 & 564.0 & 19.57 & 0.30 & - & 0.14 \\
\hline 6422 & 510.6 & 559.8 & 19.04 & 0.18 & - & 0.22 & 6461 & 371.6 & 653.9 & 19.60 & 0.31 & - & 0.33 \\
\hline 6423 & 251.7 & 476.5 & 19.04 & 0.38 & - & 0.51 & 6462 & 467.1 & 717.8 & 19.63 & 0.30 & - & 0.40 \\
\hline 6424 & 391.5 & 178.2 & 19.10 & 0.16 & - & 0.01 & 6463 & 445.3 & 368.8 & 19.66 & 0.30 & - & 0.42 \\
\hline 6425 & 480.6 & 543.1 & 19.10 & 0.21 & - & 0.48 & 6464 & 352.7 & 284.5 & 19.66 & 0.21 & - & 0.46 \\
\hline 6426 & 396.4 & 210.5 & 19.11 & 0.15 & - & 0.07 & 6465 & 578.1 & 544.7 & 19.72 & 0.28 & - & 0.31 \\
\hline 6427 & 160.7 & 706.0 & 19.11 & 0.29 & - & 0.43 & 6466 & 175.1 & 272.9 & 19.72 & 0.23 & - & 0.48 \\
\hline 6428 & 275.4 & 291.8 & 19.12 & 0.17 & - & 0.47 & 6467 & 455.5 & 382.7 & 19.72 & 0.39 & - & 0.50 \\
\hline 6429 & 254.4 & 344.3 & 19.13 & 0.20 & - & 0.20 & 6468 & 364.3 & 288.7 & 19.78 & 0.27 & - & 0.33 \\
\hline 6430 & 662.0 & 633.7 & 19.13 & 0.15 & -1.15 & 0.44 & 6469 & 681.5 & 515.7 & 19.79 & 0.32 & - & 0.74 \\
\hline 6431 & 227.7 & 646.3 & 19.14 & 0.19 & - & 0.13 & 6470 & 102.6 & 769.4 & 19.80 & 0.27 & - & 0.13 \\
\hline 6432 & 669.8 & 711.0 & 19.15 & 0.15 & - & 0.50 & 6471 & 90.4 & 461.2 & 19.84 & 0.29 & - & 0.82 \\
\hline 6433 & 469.9 & 527.3 & 19.16 & 0.21 & - & 0.14 & 6472 & 446.6 & 553.5 & 19.85 & 0.35 & - & 0.26 \\
\hline 6434 & 402.7 & 650.2 & 19.17 & 0.18 & - & 0.39 & 6473 & 706.7 & 732.0 & 19.91 & 0.30 & - & 1.16 \\
\hline 6435 & 458.8 & 371.9 & 19.17 & 0.20 & - & 0.24 & 6474 & 505.2 & 599.6 & 19.95 & 0.44 & - & 0.50 \\
\hline 6436 & 249.6 & 300.2 & 19.17 & 0.15 & - & 0.51 & 6475 & 137.8 & 768.4 & 20.07 & 0.38 & - & 0.41 \\
\hline 6437 & 249.8 & 524.9 & 19.18 & 0.26 & - & 0.42 & 6476 & 371.6 & 304.1 & 20.29 & 0.40 & - & 0.63 \\
\hline 6438 & 99.7 & 177.3 & 19.20 & 0.14 & - & -0.08 & 6477 & 773.0 & 439.1 & 20.39 & 0.52 & - & 1.43 \\
\hline 6439 & 439.2 & 264.8 & 19.23 & 0.17 & - & -0.03 & & & & & & & \\
\hline
\end{tabular}


TABLE 5. Photometry of stars outside R136 (PC7)

\begin{tabular}{|c|c|c|c|c|c|c|c|c|c|c|c|c|c|}
\hline ID & $x$ & $\mathrm{y}$ & F555W & $\sigma$ & $336-555$ & $555-702$ & ID & $\mathrm{x}$ & $\mathrm{y}$ & F555W & $\sigma$ & $336-555$ & $555-702$ \\
\hline 7001 & 615.8 & 150.2 & 11.81 & 0.04 & -0.90 & 0.37 & 7041 & 365.6 & 81.9 & 15.98 & 0.05 & -1.01 & 0.18 \\
\hline 7002 & 251.8 & 187.9 & 12.57 & 0.05 & -1.04 & 0.41 & 7042 & 432.0 & 267.7 & 16.11 & 0.06 & -1.05 & 0.17 \\
\hline 7003 & 411.6 & 133.1 & 13.26 & 0.04 & -1.02 & 0.19 & 7043 & 652.2 & 464.0 & 16.12 & 0.05 & -1.11 & 0.09 \\
\hline 7004 & 77.2 & 650.7 & 13.28 & 0.03 & - & 1.87 & 7044 & 185.8 & 316.0 & 16.15 & 0.09 & -1.20 & 0.39 \\
\hline 7005 & 535.6 & 79.7 & 13.48 & 0.05 & -1.15 & 0.26 & 7045 & 443.4 & 131.7 & 16.21 & 0.09 & -1.01 & -0.01 \\
\hline 7006 & 492.0 & 367.2 & 13.61 & 0.04 & -1.11 & 0.26 & 7046 & 734.6 & 493.0 & 16.22 & 0.05 & -0.99 & 0.10 \\
\hline 7007 & 298.0 & 198.3 & 13.65 & 0.06 & -1.11 & 0.15 & 7047 & 375.6 & 233.5 & 16.25 & 0.06 & -0.88 & 0.02 \\
\hline 7008 & 602.9 & 210.0 & 13.65 & 0.04 & -0.95 & 0.16 & 7048 & 497.0 & 658.2 & 16.31 & 0.06 & -1.14 & 0.09 \\
\hline 7009 & 325.1 & 375.2 & 13.77 & 0.04 & -1.11 & 0.14 & 7049 & 214.1 & 430.2 & 16.34 & 0.06 & -0.86 & -0.02 \\
\hline 7010 & 460.2 & 88.9 & 13.92 & 0.06 & -1.32 & 0.26 & 7050 & 112.9 & 226.4 & 16.36 & 0.08 & -1.16 & 0.22 \\
\hline 7011 & 684.5 & 264.3 & 14.40 & 0.04 & -1.08 & 0.18 & 7051 & 184.3 & 184.0 & 16.39 & 0.07 & -0.35 & 0.06 \\
\hline 7012 & 299.0 & 340.9 & 14.49 & 0.05 & -1.09 & -0.09 & 7052 & 250.1 & 100.7 & 16.42 & 0.07 & -0.99 & 0.22 \\
\hline 7013 & 564.5 & 174.6 & 14.58 & 0.04 & -1.14 & 0.06 & 7053 & 348.2 & 343.0 & 16.42 & 0.08 & -0.59 & 0.14 \\
\hline 7014 & 500.6 & 77.9 & 14.65 & 0.05 & -1.21 & 0.19 & 7054 & 582.5 & 105.9 & 16.45 & 0.05 & -0.78 & 0.06 \\
\hline 7015 & 447.1 & 171.5 & 14.66 & 0.05 & -1.32 & 0.20 & 7055 & 196.9 & 207.8 & 16.47 & 0.07 & -0.79 & 0.20 \\
\hline 7016 & 461.3 & 230.5 & 14.72 & 0.05 & -1.21 & 0.19 & 7056 & 707.2 & 684.2 & 16.47 & 0.06 & -0.99 & 0.10 \\
\hline 7017 & 286.1 & 155.1 & 14.80 & 0.07 & -1.07 & 0.15 & 7057 & 545.4 & 196.2 & 16.49 & 0.06 & -0.51 & 0.13 \\
\hline 7018 & 297.5 & 262.0 & 14.83 & 0.05 & -1.11 & 0.09 & 7058 & 114.2 & 142.9 & 16.50 & 0.10 & -0.79 & 0.10 \\
\hline 7019 & 402.8 & 138.2 & 14.91 & 0.06 & -1.06 & 0.08 & 7059 & 515.2 & 419.6 & 16.52 & 0.06 & -1.09 & 0.20 \\
\hline 7020 & 186.6 & 142.8 & 14.93 & 0.07 & -0.85 & 0.25 & 7060 & 353.1 & 264.3 & 16.60 & 0.06 & -1.04 & 0.27 \\
\hline 7021 & 128.4 & 77.6 & 14.94 & 0.06 & -1.00 & 0.29 & 7061 & 536.0 & 282.1 & 16.62 & 0.05 & -1.10 & 0.13 \\
\hline 7022 & 466.2 & 455.3 & 15.04 & 0.04 & -1.13 & 0.14 & 7062 & 373.9 & 52.3 & 16.65 & 0.06 & -0.91 & 0.19 \\
\hline 7023 & 407.9 & 345.0 & 15.08 & 0.05 & -1.23 & 0.22 & 7063 & 674.5 & 343.1 & 16.67 & 0.04 & -0.71 & 0.12 \\
\hline 7024 & 324.5 & 580.5 & 15.10 & 0.04 & -1.14 & 0.15 & 7064 & 564.9 & 43.4 & 16.68 & 0.08 & -0.69 & 0.29 \\
\hline 7025 & 660.6 & 55.3 & 15.12 & 0.05 & -1.01 & 0.19 & 7065 & 144.4 & 179.1 & 16.72 & 0.11 & -0.51 & 0.12 \\
\hline 7026 & 663.4 & 224.9 & 15.22 & 0.04 & -1.04 & 0.09 & 7066 & 67.6 & 99.3 & 16.74 & 0.08 & -0.53 & 0.22 \\
\hline 7027 & 389.5 & 194.3 & 15.27 & 0.05 & -0.89 & 0.09 & 7067 & 757.3 & 718.5 & 16.74 & 0.07 & -1.22 & 0.05 \\
\hline 7028 & 589.5 & 217.1 & 15.28 & 0.05 & -1.04 & 0.08 & 7068 & 394.8 & 379.9 & 16.74 & 0.06 & -1.01 & 0.19 \\
\hline 7029 & 125.5 & 67.6 & 15.34 & 0.10 & -0.71 & 0.40 & 7069 & 499.7 & 101.2 & 16.80 & 0.10 & -0.89 & 0.00 \\
\hline 7030 & 483.7 & 408.7 & 15.39 & 0.04 & -1.09 & -0.06 & 7070 & 596.7 & 84.0 & 16.83 & 0.05 & -0.49 & 0.04 \\
\hline 7031 & 709.5 & 483.5 & 15.42 & 0.05 & -1.12 & 0.14 & 7071 & 656.2 & 274.0 & 16.89 & 0.08 & -0.56 & 0.04 \\
\hline 7032 & 417.1 & 178.8 & 15.49 & 0.07 & -1.19 & 0.16 & 7072 & 606.4 & 249.7 & 16.91 & 0.09 & -0.75 & 0.12 \\
\hline 7033 & 721.0 & 406.8 & 15.51 & 0.06 & -1.00 & 0.19 & 7073 & 624.0 & 260.2 & 16.91 & 0.06 & -0.77 & 0.18 \\
\hline 7034 & 206.0 & 349.2 & 15.51 & 0.07 & -1.19 & 0.29 & 7074 & 722.1 & 760.9 & 16.92 & 0.08 & -0.92 & 0.15 \\
\hline 7035 & 375.2 & 195.8 & 15.52 & 0.05 & -1.03 & 0.60 & 7075 & 157.1 & 66.2 & 17.01 & 0.08 & -0.67 & 0.12 \\
\hline 7036 & 211.1 & 481.3 & 15.57 & 0.06 & -1.06 & 0.08 & 7076 & 546.1 & 654.0 & 17.05 & 0.08 & -1.09 & 0.07 \\
\hline 7037 & 617.2 & 269.5 & 15.89 & 0.05 & -1.13 & 0.25 & 7077 & 413.2 & 211.8 & 17.06 & 0.10 & -0.74 & 0.09 \\
\hline 7038 & 188.7 & 312.3 & 15.93 & 0.07 & -0.97 & 0.19 & 7078 & 759.7 & 354.7 & 17.07 & 0.06 & -0.61 & 0.17 \\
\hline 7039 & 561.7 & 119.6 & 15.93 & 0.05 & -1.03 & 0.08 & 7079 & 414.7 & 281.6 & 17.10 & 0.08 & -0.80 & 0.40 \\
\hline 7040 & 709.1 & 631.6 & 15.96 & 0.06 & -1.03 & 0.01 & 7080 & 166.4 & 153.6 & 17.10 & 0.15 & - & 0.21 \\
\hline 7081 & 662.4 & 482.9 & 17.10 & 0.07 & -1.02 & -0.03 (b) & 7121 & 483.6 & 230.1 & 17.94 & 0.12 & -1.01 & 0.13 \\
\hline 7082 & 637.7 & 67.4 & 17.16 & 0.06 & -0.84 & 0.07 & 7122 & 201.6 & 358.2 & 17.94 & 0.16 & -0.93 & 0.09 \\
\hline 7083 & 365.8 & 59.8 & 17.19 & 0.08 & -0.88 & 0.15 & 7123 & 678.4 & 254.5 & 17.94 & 0.16 & -0.42 & 0.09 \\
\hline 7084 & 98.8 & 79.6 & 17.22 & 0.09 & - & 0.05 & 7124 & 438.6 & 210.2 & 17.95 & 0.14 & - & 0.16 \\
\hline 7085 & 140.7 & 262.3 & 17.25 & 0.10 & -0.89 & 0.29 & 7125 & 473.0 & 557.6 & 17.97 & 0.09 & -0.86 & -0.08 \\
\hline 7086 & 551.5 & 97.8 & 17.25 & 0.10 & -0.81 & -0.12 & 7126 & 154.9 & 118.1 & 17.98 & 0.23 & - & 0.28 \\
\hline 7087 & 599.9 & 480.5 & 17.29 & 0.06 & -0.75 & 0.00 & 7127 & 335.6 & 552.7 & 18.01 & 0.12 & -1.54 & 0.26 \\
\hline 7088 & 205.4 & 198.4 & 17.30 & 0.16 & -0.29 & 0.15 & 7128 & 301.6 & 499.1 & 18.06 & 0.11 & -1.30 & 0.32 \\
\hline 7089 & 314.3 & 703.8 & 17.33 & 0.08 & -1.10 & -0.02 & 7129 & 483.3 & 123.3 & 18.12 & 0.23 & -0.72 & -0.07 \\
\hline 7090 & 622.8 & 341.0 & 17.33 & 0.06 & -0.83 & 0.13 & 7130 & 200.3 & 324.7 & 18.27 & 0.20 & - & 0.13 \\
\hline 7091 & 577.4 & 559.1 & 17.35 & 0.07 & -1.03 & -0.03 & 7131 & 615.6 & 627.7 & 18.32 & 0.14 & -1.14 & 0.02 \\
\hline 7092 & 367.7 & 308.4 & 17.39 & 0.11 & -0.45 & 0.02 & 7132 & 716.6 & 338.1 & 18.35 & 0.12 & -0.54 & 0.24 \\
\hline 7093 & 496.3 & 539.9 & 17.41 & 0.08 & -1.10 & 0.22 & 7133 & 453.9 & 373.3 & 18.36 & 0.20 & - & 0.38 \\
\hline 7094 & 768.2 & 293.7 & 17.46 & 0.07 & -0.96 & 0.11 & 7134 & 623.6 & 208.0 & 18.37 & 0.28 & - & 0.51 \\
\hline 7095 & 365.7 & 52.2 & 17.47 & 0.09 & -0.60 & 0.13 & 7135 & 716.8 & 721.8 & 18.40 & 0.18 & - & 0.14 \\
\hline 7096 & 190.1 & 465.1 & 17.48 & 0.11 & -0.86 & 0.21 & 7136 & 157.7 & 94.4 & 18.41 & 0.30 & - & 0.05 \\
\hline 7097 & 377.7 & 61.0 & 17.49 & 0.10 & -0.83 & 0.06 & 7137 & 644.0 & 463.2 & 18.45 & 0.15 & -1.07 & 0.21 \\
\hline 7098 & 342.2 & 299.3 & 17.50 & 0.08 & - & 0.10 & 7138 & 368.4 & 385.0 & 18.50 & 0.29 & - & 0.08 \\
\hline 7099 & 510.6 & 244.4 & 17.50 & 0.08 & -0.60 & 0.20 & 7139 & 74.0 & 499.0 & 18.51 & 0.13 & - & 0.18 \\
\hline 7100 & 506.5 & 172.1 & 17.52 & 0.10 & -0.86 & 0.11 & 7140 & 47.8 & 520.5 & 18.51 & 0.13 & - & 0.20 \\
\hline 7101 & 390.8 & 48.7 & 17.53 & 0.09 & -0.71 & 0.21 & 7141 & 162.2 & 465.1 & 18.55 & 0.19 & - & 0.22 \\
\hline 7102 & 647.3 & 110.6 & 17.59 & 0.07 & - & 0.05 & 7142 & 536.5 & 593.0 & 18.60 & 0.19 & - & -0.07 \\
\hline 7103 & 727.5 & 579.9 & 17.60 & 0.07 & - & 0.55 & 7143 & 377.1 & 564.5 & 18.66 & 0.16 & - & 0.16 \\
\hline 7104 & 495.9 & 198.9 & 17.60 & 0.10 & -1.06 & 0.13 & 7144 & 553.0 & 136.5 & 18.70 & 0.23 & - & 0.14 \\
\hline 7105 & 681.5 & 401.8 & 17.61 & 0.11 & -1.00 & 0.20 & 7145 & 188.2 & 422.7 & 18.70 & 0.21 & -1.28 & 0.16 \\
\hline 7106 & 654.5 & 284.5 & 17.70 & 0.12 & - & 0.05 & 7146 & 404.5 & 527.8 & 18.80 & 0.16 & - & -0.12 \\
\hline 7107 & 369.4 & 583.4 & 17.71 & 0.10 & - & 0.06 & 7147 & 533.2 & 472.7 & 18.81 & 0.18 & - & 0.22 \\
\hline 7108 & 225.3 & 73.8 & 17.72 & 0.11 & 0.09 & 0.25 & 7148 & 750.8 & 319.8 & 18.97 & 0.20 & - & 0.28 \\
\hline 7109 & 455.9 & 176.7 & 17.72 & 0.14 & -0.50 & 0.47 & 7149 & 455.0 & 657.3 & 19.01 & 0.26 & - & 0.04 \\
\hline 7110 & 309.8 & 442.2 & 17.73 & 0.09 & -0.66 & 0.29 & 7150 & 280.1 & 577.3 & 19.03 & 0.26 & - & 0.34 \\
\hline 7111 & 661.2 & 608.7 & 17.73 & 0.09 & - & 0.09 & 7151 & 312.1 & 469.6 & 19.08 & 0.19 & - & 0.74 \\
\hline 7112 & 552.5 & 122.4 & 17.77 & 0.13 & -0.26 & -0.19 & 7152 & 559.8 & 415.0 & 19.10 & 0.25 & - & 0.33 \\
\hline 7113 & 637.9 & 442.7 & 17.80 & 0.09 & - & 0.07 & 7153 & 589.1 & 338.8 & 19.10 & 0.23 & - & 0.28 \\
\hline 7114 & 526.3 & 177.8 & 17.80 & 0.11 & -0.28 & 0.16 & 7154 & 628.1 & 420.0 & 19.11 & 0.20 & - & -0.08 \\
\hline 7115 & 467.6 & 250.4 & 17.82 & 0.12 & -0.84 & 0.24 & 7155 & 312.3 & 725.3 & 19.15 & 0.21 & - & 0.78 \\
\hline 7116 & 451.2 & 351.6 & 17.82 & 0.17 & -0.34 & 0.19 & 7156 & 320.8 & 290.7 & 19.26 & 0.41 & - & 0.41 \\
\hline 7117 & 754.6 & 480.3 & 17.85 & 0.11 & -0.98 & 0.04 & 7157 & 583.7 & 370.0 & 19.32 & 0.28 & - & -0.22 \\
\hline 7118 & 291.8 & 90.4 & 17.91 & 0.12 & -1.02 & 0.12 & 7158 & 134.0 & 386.1 & 19.36 & 0.21 & - & 0.54 \\
\hline 7119 & 656.7 & 204.3 & 17.93 & 0.18 & - & 0.24 & 7159 & 441.9 & 263.8 & 19.73 & 0.58 & - & 0.34 \\
\hline \multirow[t]{3}{*}{7120} & 653.1 & 306.9 & 17.94 & 0.10 & -0.37 & 0.06 & 7160 & 155.1 & 141.2 & 14.27 & 0.04 & -0.78 & 0.19 \\
\hline & & & & & & & 7161 & 153.2 & 143.3 & 14.30 & 0.06 & -1.32 & 0.19 \\
\hline & & & & & & & 7162 & 139.6 & 146.0 & 14.52 & 0.04 & -1.02 & 0.07 \\
\hline
\end{tabular}


TABLE 6. Photometry of stars outside R136 (PC8).

\begin{tabular}{|c|c|c|c|c|c|c|c|c|c|c|c|c|c|}
\hline ID & $x$ & $y$ & F555W & $\sigma$ & $336-555$ & $555-702$ & ID & $\mathrm{x}$ & $\mathrm{y}$ & F555W & $\sigma$ & $336-555$ & $555-702$ \\
\hline 8001 & 618.7 & 253.0 & 14.18 & 0.03 & -1.20 & 0.18 & 8030 & 599.8 & 452.7 & 19.14 & 0.11 & - & 0.51 \\
\hline 8002 & 223.9 & 539.4 & 14.37 & 0.03 & -1.20 & 0.19 & 8031 & 123.2 & 183.2 & 19.19 & 0.11 & -1.00 & 0.29 \\
\hline 8003 & 310.2 & 477.6 & 14.77 & 0.04 & -1.08 & 0.21 & 8032 & 128.5 & 219.1 & 19.23 & 0.11 & - & 1.44 \\
\hline 8004 & 71.3 & 484.3 & 15.05 & 0.05 & -0.89 & 0.28 & 8033 & 31.4 & 222.8 & 19.32 & 0.12 & -0.91 & 0.26 \\
\hline 8005 & 793.4 & 393.6 & 15.23 & 0.05 & -1.28 & 0.12 & 8034 & 379.3 & 336.8 & 19.37 & 0.11 & - & 0.07 \\
\hline 8006 & 581.8 & 371.0 & 15.69 & 0.03 & -1.07 & 0.16 & 8035 & 429.6 & 89.7 & 19.52 & 0.17 & - & 0.76 \\
\hline 8007 & 118.7 & 47.0 & 15.75 & 0.04 & -0.78 & 0.26 & 8036 & 689.1 & 70.2 & 19.55 & 0.16 & -1.20 & 0.51 \\
\hline 8008 & 510.9 & 490.1 & 15.95 & 0.04 & -1.17 & 0.18 & 8037 & 155.9 & 547.7 & 19.73 & 0.17 & -0.92 & 0.63 \\
\hline 8009 & 291.9 & 430.3 & 16.12 & 0.03 & -1.08 & 0.18 & 8038 & 756.7 & 217.4 & 19.74 & 0.17 & - & 1.52 \\
\hline 8010 & 213.8 & 618.7 & 16.13 & 0.04 & -1.12 & 0.22 & 8039 & 533.1 & 124.2 & 19.76 & 0.19 & - & 0.48 \\
\hline 8011 & 478.6 & 63.6 & 16.83 & 0.04 & -1.01 & 0.33 & 8040 & 399.5 & 526.5 & 19.82 & 0.20 & - & 0.11 \\
\hline 8012 & 501.6 & 84.7 & 17.57 & 0.05 & -0.87 & 0.31 & 8041 & 186.6 & 241.4 & 19.85 & 0.18 & - & 0.50 \\
\hline 8013 & 481.2 & 110.6 & 17.60 & 0.05 & -0.99 & 0.27 & 8042 & 113.1 & 91.8 & 19.86 & 0.30 & - & 0.41 \\
\hline 8014 & 368.2 & 195.1 & 17.60 & 0.05 & -0.81 & 0.24 & 8043 & 37.6 & 263.3 & 19.88 & 0.21 & - & 0.17 \\
\hline 8015 & 520.7 & 196.6 & 17.65 & 0.03 & - & 0.25 & 8044 & 673.6 & 154.4 & 19.90 & 0.17 & - & 0.98 \\
\hline 8016 & 209.1 & 289.4 & 17.69 & 0.05 & -0.79 & 0.32 & 8045 & 457.8 & 96.9 & 19.94 & 0.27 & - & 0.25 \\
\hline 8017 & 238.1 & 78.8 & 17.70 & 0.05 & -0.79 & 0.36 & 8046 & 496.5 & 577.5 & 19.95 & 0.23 & - & 0.34 \\
\hline 8018 & 321.9 & 377.4 & 17.75 & 0.05 & -0.78 & 0.26 & 8047 & 297.2 & 53.1 & 19.96 & 0.25 & - & 1.29 \\
\hline 8019 & 695.2 & 222.7 & 17.89 & 0.06 & -0.89 & 0.25 & 8048 & 556.0 & 603.6 & 19.98 & 0.21 & - & 0.34 \\
\hline 8020 & 578.5 & 525.7 & 17.91 & 0.05 & -0.62 & 0.34 & 8049 & 505.0 & 479.0 & 19.98 & 0.33 & 1.10 & 0.68 \\
\hline 8021 & 565.5 & 221.1 & 18.10 & 0.05 & -0.83 & 0.32 & 8050 & 629.8 & 693.2 & 20.05 & 0.23 & - & 1.09 \\
\hline 8022 & 31.1 & 128.6 & 18.14 & 0.06 & -0.45 & 0.23 & 8051 & 319.2 & 330.6 & 20.07 & 0.25 & - & 0.51 \\
\hline 8023 & 256.6 & 66.9 & 18.28 & 0.06 & -0.74 & 0.34 & 8052 & 767.9 & 116.9 & 20.13 & 0.29 & - & 0.74 \\
\hline 8024 & 362.7 & 224.6 & 18.53 & 0.07 & -0.12 & 0.36 & 8053 & 100.5 & 201.6 & 20.14 & 0.25 & - & 0.51 \\
\hline 8025 & 457.1 & 186.1 & 18.60 & 0.09 & - & 1.01 & 8054 & 435.7 & 250.6 & 20.17 & 0.22 & - & 0.42 \\
\hline 8026 & 511.7 & 322.4 & 18.72 & 0.07 & -0.88 & 0.15 & 8055 & 711.2 & 670.2 & 20.22 & 0.21 & - & 0.82 \\
\hline 8027 & 472.1 & 214.6 & 18.80 & 0.08 & -1.05 & 0.34 & 8056 & 123.1 & 107.2 & 20.28 & 0.41 & - & 0.89 \\
\hline 8028 & 107.1 & 618.5 & 18.82 & 0.10 & -0.45 & 0.18 & 8057 & 448.6 & 548.0 & 20.28 & 0.34 & - & 0.34 \\
\hline 8029 & 230.7 & 171.3 & 19.05 & 0.08 & - & 0.40 & 8058 & 378.3 & 138.8 & 20.99 & 0.55 & - & 1.67 \\
\hline
\end{tabular}

R136 are very similar. In the $M_{336}-M_{555}$ color, however, there appears to be a small, $\sim 0.05$ to $0.1 \mathrm{mag}$, offset in the loci of points such that the R136 stars are slightly redder than stars outside of R136. However, this offset is primarily an offset between CCD chips. That is, the stars within R136 and outside of R136 but on PC6 fall in the same area of the diagram, but both are offset from the stars outside of R136 that fall on PCs 5, 7, and 8 although PCs 5 and 8 are sparsely populated. If this offset is due to a difference in reddening, it would imply that the stars on PC6 are undercorrected for reddening relative to the stars on PCs 5, 7, and 8 which are further away from the core. This is possible but counterintuitive since one might expect the interstellar medium within the R136 region to be more cleared out due to the winds from the concentration of massive stars. A more likely explanation is that the offset is due to the host of difficulties in cross-normalizing the flat-fields among the CCD chips. The flat-fielding problems can cause effects of this order of magnitude. The WF/PC Investigation Definition Team (1992; see Chaps. 6 and 12) gives a detailed discussion of this problem. Therefore, with the current data we cannot be sure that the offset that is seen in the loci of stars in the color-magnitude diagram is real.

\subsection{The Luminosity Function}

We have counted stars in 0.5 mag luminosity bins for R136 and for the area outside. The $\log$ of the luminosity function is shown in Fig. 11, and the log of the cumulative luminosity function is shown in Fig. 12. One can see that the slopes of the luminosity functions within and without R136 are very similar between $M_{555}=-7$ and -4 . Fainter than $M_{555}=-4$, the star counts within R136 clearly be- come incomplete; it is harder to detect and measure faint stars in the crowded region of R136 than outside of it.

We estimated incompleteness by using the automatic star finding technique mentioned in Sec. 2.2. One hundred artificial stars per magnitude bin were placed on the F555W and F702W frames, one at a time, in annuli centered on R136 with widths of 20 pixels. For each radius and each magnitude bin, incompleteness was estimated by seeing how many of the 100 stars were found and measured by the automatic routine. We then applied these corrections to the raw luminosity function. Results are presented in Fig. 13 for stars within 40 pixels of R136 and for stars outside of this region. The number of stars in the outer region was normalized to match approximately the number found in the inner region. With this completeness correction, the apparent turnover seen in Fig. 11 for the center of R136 goes away. Fainter than $M_{V} \sim-3$, it is impossible to estimate completeness corrections in the center of R136 because essentially none of the artificial stars were found. Figure 13 shows that there is a slight hint that there may be more massive stars relative to lower mass stars inside of R136 than outside the central region. However, the differences are within the estimated errors, so we do not take this as strong evidence for mass segregation. We hope to explore this question in more detail with higher signal-tonoise data to be obtained in the future. We note that mass segregation from dynamical effects would be expected only for the very central region of the cluster. Mass segregation resulting from a variable initial mass function, however, could occur anywhere.

For the range $M_{555}=-4$ to -7 , the slope $\delta(\log N) /$ $\delta M_{V}$ of the luminosity function is 0.3 . No significant contamination by Galactic foreground stars is expected in the 


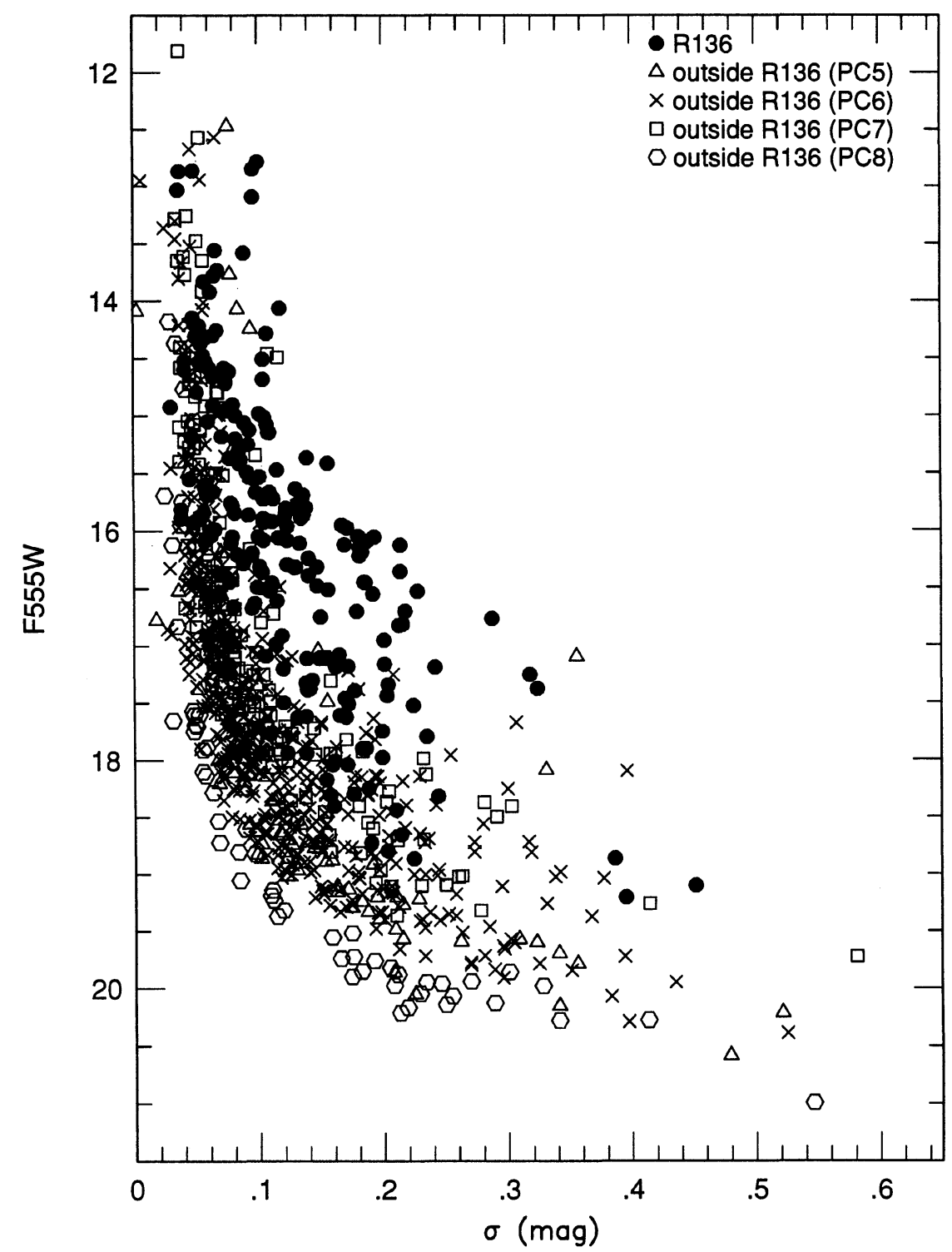

FIG. 5. The uncertainty, $\sigma$, in the magnitudes as a function of the magnitudes for filter F555W. Stars within R136 are shown with filled circles and those without are triangles (PC5), X's (PC6), squares (PC7), and hexagons (PC8). Stars with $\sigma>0.6$ have been eliminated from the star lists. Clearly, stars within the more crowded R136 region have a higher uncertainty for a given magnitude than those stars outside of the core.

direction of the LMC (Ratnatunga \& Bahcall 1985). This slope can be compared with that measured from the luminosity function plotted by Melnick (1986) for stars in 30 Doradus but outside R136. For the same luminosity range his slope is $\sim 0.26$, which is in very good agreement with ours for the core of the region. This would imply that the luminosity function is similar throughout 30 Doradus (but see Parker 1992). Unfortunately, without spectral classifications for the stars in R136 we are unable to translate the luminosity function into a true mass function (Massey 1985). Melnick, however, uses a relationship between $M_{V}$ and ZAMS mass for a star cluster of 2 Myrs to convert his luminosity function to a mass function. From this he determines a mass function slope of $1.0 \pm 0.3$ where a Salpeter function has a slope of 1.35 .

Another region of star formation that was undoubtedly once comparable to 30 Doradus in terms of overall intensity of star formation is Constellation III, located to the north. Reid et al. (1987) examined the $V$-band luminosity function for that region and found a slope of 0.55 . This slope is similar to that compiled by Scalo (1986) for the Milky Way field stars and by Freedman (1985) for a large sample of nearby galaxies. Thus, the slope of the luminosity function that we find for R136 appears to be shallower 
TABLE 7. Identification of stars.

\begin{tabular}{lll}
\hline \hline ID from Literature & & \\
\hline Mk5 & ID in Tables 2-6 & Spectral type $^{b}$ \\
Mk6 & 5003 & B2: Ip? \\
Mk7 & 8002 & O8-9 \\
Mk8 & 8003 & O(7) V \\
Mk9 & 8001 & O5-6 \\
Mk12 & 7004 & - \\
Mk13 & 5001 & B0-0.5 Ia \\
Mk14 & 5104 & O3-4 \\
Mk14N & 5004 & O3-6 V \\
Mk15 & 6020 & O V \\
Mk15S & $7160,7161,7162$ & O7 V \\
Mk24 & 7021,7029 & O3-6 V \\
Mk26 & 7009 & O3-4 V \\
Mk30 & 7006 & O3-4 V \\
Mk32 & 7005 & O3 If/WN6-A \\
Mk33S & 7003,7019 & O8 II \\
Mk33N & 6009,6013 & - \\
Mk34 & 6008,6014 & - \\
Mk35 & 6004 & WN4.5 \\
Mk35N & 6006 & O3 If/WN6-A \\
Mk35S & $6017,6089,6143,6161,6264$ & O5-7: \\
Mk37 & 6010 & O5-6 V \\
Mk37W & 6007 & WN7-A \\
Mk39 & 6005 & - \\
Mk42 & 6003 & O3 If/WN6-A \\
R134 & 6002 & O3 If/WN6-A \\
R136b & 6001 & WN7 \\
R136c & 26 & - \\
R141 & 25 & B0.5 I \\
R142 & 7002. & B0.5-0.7 I \\
\hline \hline
\end{tabular}

NOTE.-

${ }^{a}$ From Melnick (1985) or Feitzinger et al. (1980).

${ }^{b}$ From Walborn (1986).

than the slope found for Constellation III; more higher mass stars are present in R136. However, this difference in slope is probably due to a difference in the evolutionary state of the regions rather than an actual initial mass function difference. Constellation III is a much older region, and Reid et al. find a main-sequence turnoff at about $M_{V} \sim-3$. In fact Reid et al. compiled a luminosity function for the ring around Constellation III where star formation is in progress today. For that young region the

TABLE 8. Emission-line stars.

\begin{tabular}{|c|c|c|c|c|}
\hline star & $\mathrm{m}(469)-\mathrm{m}_{R 134}(469)^{n}$ & $\sigma$ & $\mathrm{ID}^{6}$ & type ${ }^{c}$ \\
\hline 1 & 0.55 & 0.06 & R136a-AS & - \\
\hline 2 & 0.66 & 0.07 & R136a-AN & - \\
\hline 3 & 0.40 & 0.04 & R136a-B & - \\
\hline 25 & 0.56 & 0.01 & R136c & - \\
\hline 26 & 2.16 & 0.04 & R136b & - \\
\hline 6001 & 0.00 & 0.01 & R134 & WN7 \\
\hline 6002 & 3.84 & 0.11 & Mk42 & O3If/WN6-A \\
\hline 6003 & 5.03 & 0.34 & Mk39 & O3If/WN6-A \\
\hline 6004 & 0.90 & 0.01 & Mk34 & WN 4.5 \\
\hline 6005 & 3.67 & 0.09 & Mk37W & WN7-A (Mk37) \\
\hline 6006 & 3.41 & 0.08 & Mk35 & O3If/WN6-A \\
\hline 6007 & 3.19 & 0.06 & Mk37 & WN7.A \\
\hline 6013 & 0.64 & 0.01 & Mk33S & WC5+O4 (Mk33) \\
\hline 6018 & 3.43 & 0.08 & - & - \\
\hline 7002 & 4.53: & 0.18 & R141 & B05.I \\
\hline 7003 & 5.51: & 0.44 & Mk32 & O8II \\
\hline 7005 & 2.65 & 0.04 & $\mathrm{Mk} 30$ & O3If/WN6-A \\
\hline 7006 & 4.62: & 0.19 & Mk26 & 03-4V \\
\hline 7009 & 4.19: & 0.14 & Mk24 & O3-4V \\
\hline 8001 & 3.27 & 0.04 & Mk8 & $05-6$ \\
\hline 8002 & 3.33 & 0.04 & Mk6 & O8-9 \\
\hline 8003 & 4.09: & 0.07 & Mk7 & $\mathrm{O}(7) \mathrm{V}$ \\
\hline 8004 & 4.26: & 0.09 & Sat & - \\
\hline
\end{tabular}

NOTE.-

${ }^{a}$ Magnitudes measured in the net F469N image (on-band minus continuum) relative to the magnitude of R134. A colon following the magnitude means that the residual is uncertain. "R136a-A and R136a-B refer to Moffat et al.'s (1985) designations. The " $N$ " and "S" appended to R136a-A indicate the northern and southern subcomponent respectively.

'From Walborn's (1986) review.

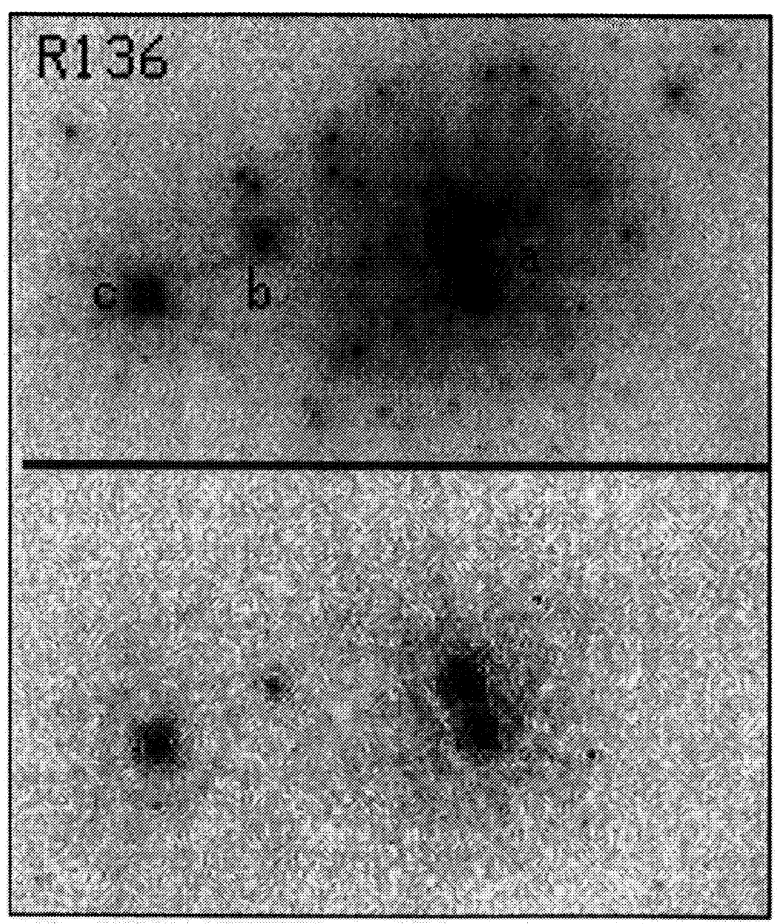

FIG. 7. An enlarged version of Fig. 6 showing only R136a, b, and c (Feitzinger et al. 1980). The top panel is the continuum image, and the bottom panel is the net emission image. One can see that the upper component of $\mathrm{R} 136 \mathrm{a}$ is itself elongated.

slope of the luminosity function is much flatter, with a slope much more like that which we measure for R136.

\subsection{Wolf-Rayet Stars}

Several ground-based studies have recognized that R136a had spectral features characteristic of Wolf-Rayet stars and was likely to contain one or more WN-type stars (cf., Walborn 1973; Moffat \& Seggewiss 1983; Chu et al. 1984; Melnick 1985; Moffat et al. 1985). The net F469N emission image, shown in Figs. 6 and 7, confirms this prediction. $\mathrm{R} 136 \mathrm{a}$ is resolved by the $\mathrm{PC}$ into three components. Star number 3 is Moffat et al.'s R136a-B, and stars 1 and 2 are their R136a-A. In addition to R136a, two other Wolf-Rayet stars were detected in R136: R136b and R136c. R136c was already known to be a Wolf-Rayet star, while R136b was not. Chu et al. (1984) did not detect $\mathrm{R} 136 \mathrm{~b}$ and stated that it must be at most a weak-lined Wolf-Rayet star or a transitional type. Although we have detected it in our emission image, $R 136 \mathrm{~b}$ is indeed much fainter than stars like R134, R136c, and the R136a's and therefore is indeed likely to be a weak-linked Wolf-Rayet or transitional stellar type. Outside of R136 we detected additional stars, most of known spectral type. The stars detected on the F469N net-emission image are listed in Table 8, and magnitudes measured from this image relative to R134 are given. The presence of the Wolf-Rayet stars 


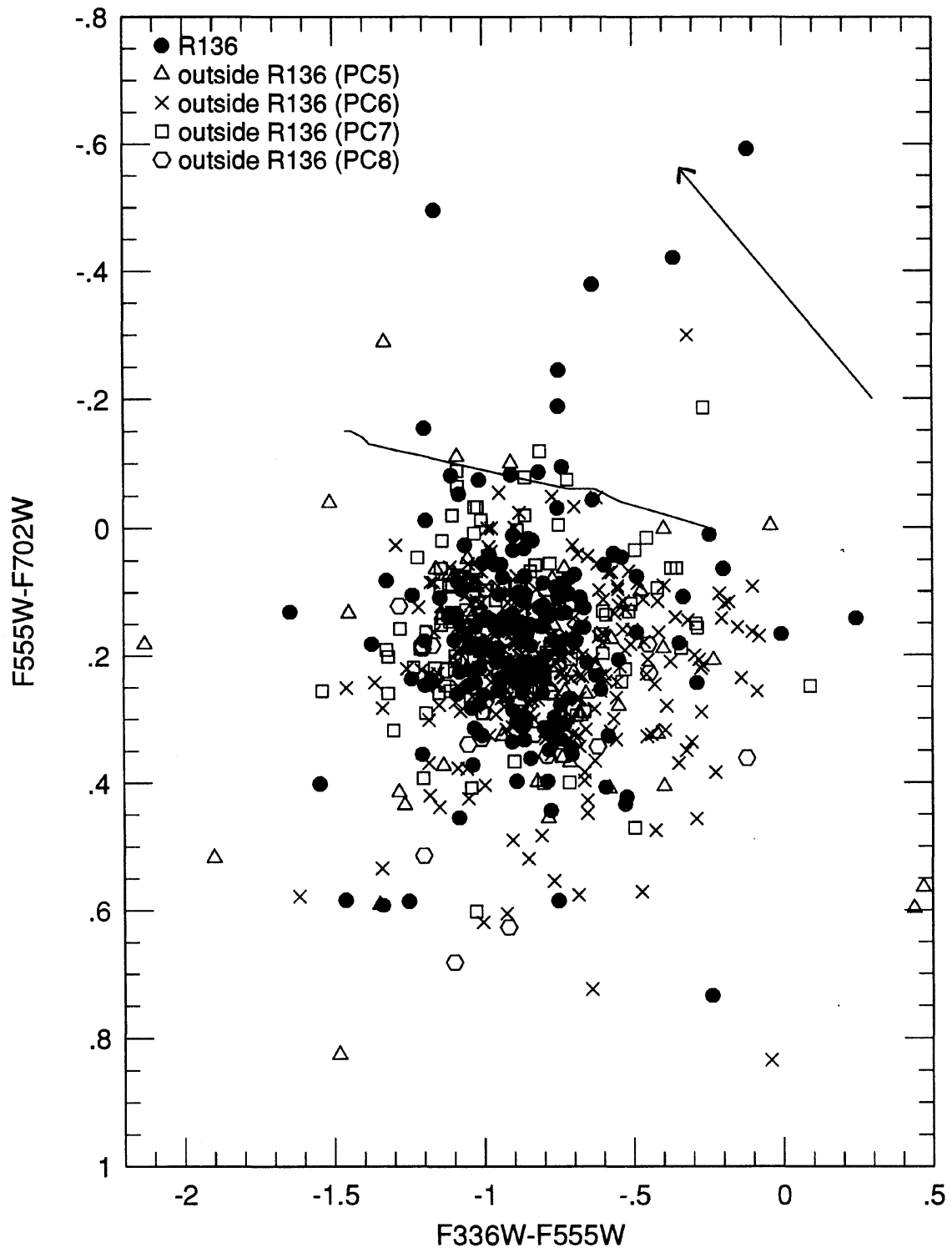

Fig. 8. A F336W-F555W vs F555W-F702W color-color diagram for the stars listed in Tables 2 through 6 . The colors have not been corrected for reddening. The straight line in the upper right is a reddening line for $\mathrm{E}(B-V)=0.34$; the curved line designates the intrinsic UVR colors for $\mathrm{O}$ and $\mathrm{B}$ stars in the Johnson system (Johnson 1968).

argues that R136a formed at least $3.5 \times 10^{6} \mathrm{yrs}$ ago, since that is the time it takes a massive star to evolve to that stage (Maeder \& Meynet 1987).

\section{$3.5 M k 5$}

The star Mk5 is classified by Walborn (1986) as " $\mathrm{B} 2$ : Ip?." Given the peculiar nature of the spectrum, we examined the PC5 image for any evidence that the star might be multiple. The image of Mk5 is shown in Fig. 14. No bright companion can be seen within several arcseconds. There is a star 1.2" southwest of Mk5, but it is $4.8 \mathrm{mag}$ fainter in F702W and does not show up in the F336W image. There- fore, the reason for the peculiar nature of the spectrum remains unknown.

\section{STELLAR DENSITIES AND THE SURFACE BRIGHTNESS PROFILE OF THE CLUSTER}

The F336W surface brightness profile for $\mathrm{R} 136$ is shown in Fig. 15 and listed in Table 9. The profile was generated by binning the stellar photometry given in Tables 2 and 4 . The center was calculated from the luminosity centroid of stars contained within the innermost arcsecond of R136 and is at $(346.26,467.40)$ in the system of coordinates given in the tables. For the few stars in the inner $10^{\prime \prime}$ that were detected in F555W but not F336W, a $U-V$ color of 


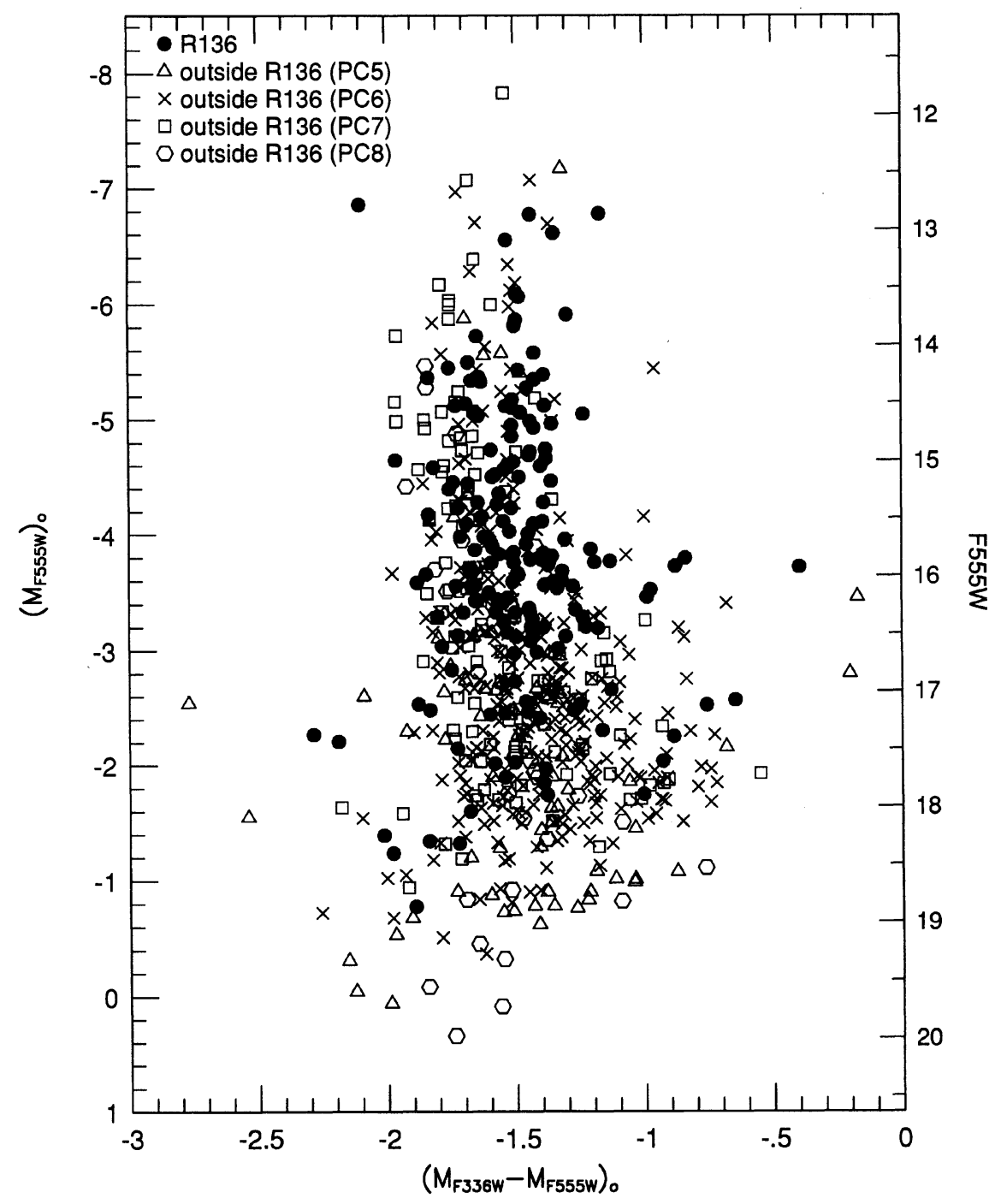

FIG. 9. A color-magnitude diagram with F336W-F555W vs F555W for the stars listed in Tables 2 through 6. The absolute, reddening-corrected magnitude is given on the left vertical axis; the apparent magnitude on the right. The colors have been corrected for reddening.

-0.80 has been assumed. The points shown in Fig. 15 and Table 9 have been corrected for absorption. The error bars reflect the luminosity weighted Poisson statistics of the number of stars in a given radial bin.

Figure 15 shows that the distribution of stars in R136 is consistent with a pure power law of the form $I(r) \propto r^{\gamma}$ with $\gamma=-1.72 \pm 0.06$. Description of the innermost structure of $\mathrm{R} 136$ is problematic, however, given the small number of stars and poor statistics at the very center. We thus also consider the possibility that the brightness profile might really have a small core, that is a central region of the profile that has $\gamma \rightarrow 0$ as $r \rightarrow 0$. Experiments with profiles including a central core lead us to conclude that the core radius, or half-power point, of the profile has $r_{c}<0$ " 25 or $0.06 \mathrm{pc}$; a profile with $r_{c}=0$ ". 25 is shown in Fig. 15 for comparison. This value of $r_{c}$ is significantly smaller than the upper limit of 0.82 " obtained by Moffat et al. (1985) from ground-based data.

Estimating the central mass density from the brightness profile can only be done crudely as we must, without the benefit of spectral classifications, estimate individual stellar masses. However, we are only interested in rough numbers for comparison with other objects, and we have made assumptions that should err on the conservative side so that the total masses and luminosities will be lower limits. We assumed a luminosity class based on the absolute magnitude $M_{555}$ of each star and determined spectral types, bolometric corrections, and masses from Conti \& Underhill (1988), Morton \& Adams (1968), and Flower (1977). Thus, we have made the following assumptions for stars in R136a and R136: (1) $M_{V}=-6.5$ to -6 : Wolf-Rayet or supergiant stars, $40 \mathscr{M}_{\odot}, \mathrm{BC}=-4:$ (2) $M_{V}=-6$ to -5.5 : 


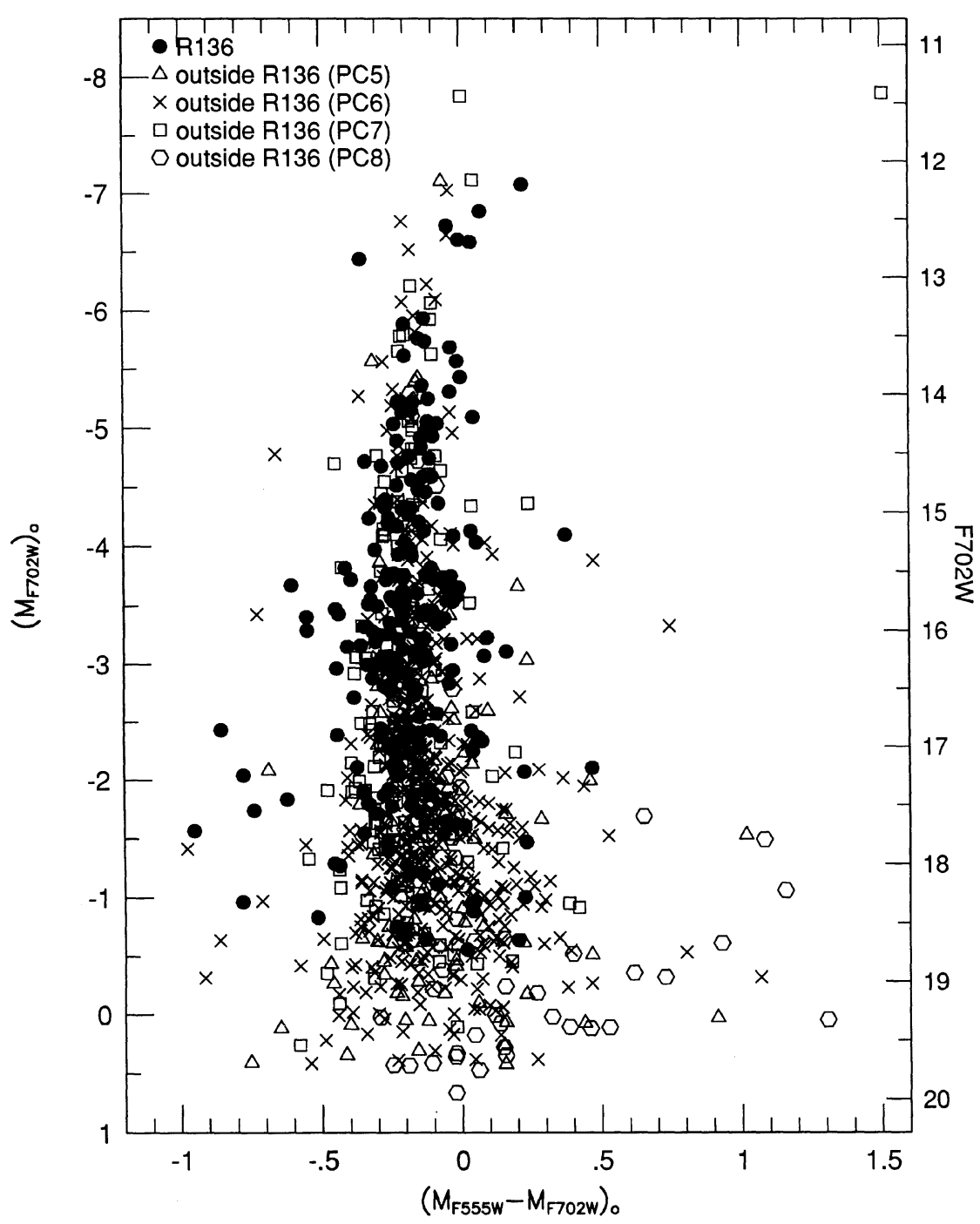

Fig. 10. A color-magnitude diagram with F555W-F702W vs F702W for the stars listed in Tables 2 through 6 . The absolute, reddening-corrected magnitude is given on the left vertical axis; the apparent magnitude on the right. The colors have been corrected for reddening. The star in the upper right corner of the plot is Mk9, an MI star (Melnick 1985).

O6.5 III, $30 \mathscr{M}_{\odot} ;$ (3) $M_{V}=-5.5$ to -5 : O9 III, $19 \mathscr{M}_{\odot}$; (4) $M_{V}=-5$ to $-4.5: 07.5 \mathrm{~V}, 25 \mathscr{M}_{\odot}$; (5) $M_{V}=-4.5$ to -4: O9 V, $19 \mathscr{M}_{\odot} ;$ (6) $M_{V}=-4$ to $-3.5: \mathrm{B} 0.5 \mathrm{~V}, 10 \mathscr{M}_{\odot}$; (7) $M_{V}=-3.5$ to -3 : B0 ZAMS, $12 \mathscr{M}_{\odot}$; (8) $M_{V}=-3$ to -2.5 ; B0.5 ZAMS, $10 \mathscr{M}_{\odot} ;(9) M_{V}=-2.5$ to -2 : B1 ZAMS; $8 \mathscr{M}_{\odot}$; (10) $M_{V}=-2$ to $-1.5:$ B2 ZAMS, $7 \mathscr{M}_{\odot}$; (11) $M_{V}=-1.5$ to $-1:$ B3 ZAMS, $5.5 \mathscr{M}_{\odot}$; and (12) $M_{V}=-1$ to $-0.5:$ B4 ZAMS, $4.3 \mathscr{M}_{\odot}$.

For $r_{c}<0.25$, the implied central mass density is $\rho_{0}>5$ $\times 10^{4} \mathscr{M}_{\odot} / \mathrm{pc}^{3}$, using the star masses given above. This is consistent with Moffat et al.'s (1985) estimate of $\sim 10^{5}$ $\mathscr{M}_{\odot} \mathrm{pc}^{-3}$ for the inner $1.5^{\prime \prime}$ and is $7 \times 10^{5}$ times the stellar mass density in the solar neighborhood. This mass, of course, includes only those stars that we have detected and whose brightnesses we have measured. Whether additional lower mass stars have been formed in the same region, to produce a "normal" initial mass function, we do not know.
In addition we estimate the total luminosity to be $\geqslant 8 \times 10^{6}$ $L_{\odot}$, where $L_{\odot}$ is the bolometric luminosity of the sun. The luminosity per unit area is $\geqslant 2 \times 10^{8} L_{\odot} \mathrm{pc}^{-2}$, which is $\sim 50$ times the luminosity per square parsec in the inner $0.2 \mathrm{pc}$ of the Orion Molecular Cloud heated by the Trapezium stars (Thronson et al. 1986).

We have also estimated the mass and luminosity of the core of NGC 3603, the most massive optically visible starforming region in the Milky Way. We used the spectral classifications given by Moffat (1983) for components A through $\mathrm{G}$, and the numbers and magnitude differences of subcomponents of $\mathrm{A}$ from speckle observations (Baier et al. 1985). Estimating the masses and luminosities as for $\mathrm{R} 136 \mathrm{a}$, we find that, compared to the $0.34 \mathrm{pc}$ region at the heart of NGC 3603, R136a has roughly three times as much stellar mass per unit volume and seven times the luminosity per unit area. 


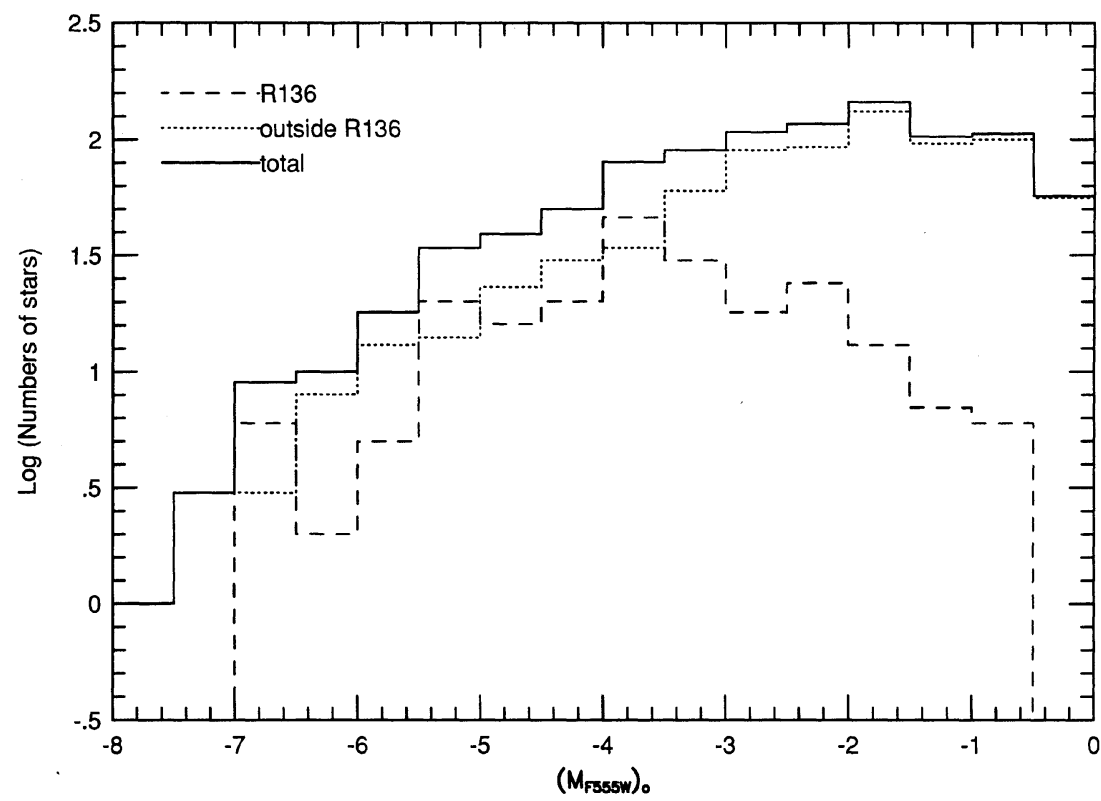

FIG. 11. The log of the luminosity function for the absolute, reddening-corrected F555W magnitude. Stars from Tables 2 through 6 have been placed in 0.5 mag bins.

We have also made estimates of the mass density and luminosity for the 214 stars in R136 using the assumptions of stellar masses given above. In the $1.9 \times 2.2 \mathrm{pc}$ region we define as R136, we total $\geqslant 3000 \mathscr{M}_{\odot}$ and $\geqslant 1.5 \times 10^{7} L_{\odot}$. The average density is $\geqslant 360 \mathscr{M}_{\odot} \mathrm{pc}^{-3}$, which is $\geqslant 6000$ times the stellar density of the solar neighborhood. The luminosity density is $\geqslant 4 \times 10^{6} L_{\odot} \mathrm{pc}^{-2}$, which is about the same as the luminosity density of the center $0.2 \mathrm{pc}$ of Orion. In other words, R136 has the same concentration of stellar luminosity as in the core of Orion, but in R136 it continues over 130 times the area.

Because the limits on $r_{c}$ are fairly small and $\rho_{0}$ is fairly large in absolute terms, it is possible that relaxation effects might be important in $\mathrm{R} 136$. The relaxation time is given by

$$
t_{r}=\frac{0.34 \sigma^{3}}{G^{2} m \rho \ln (0.4 N)},
$$

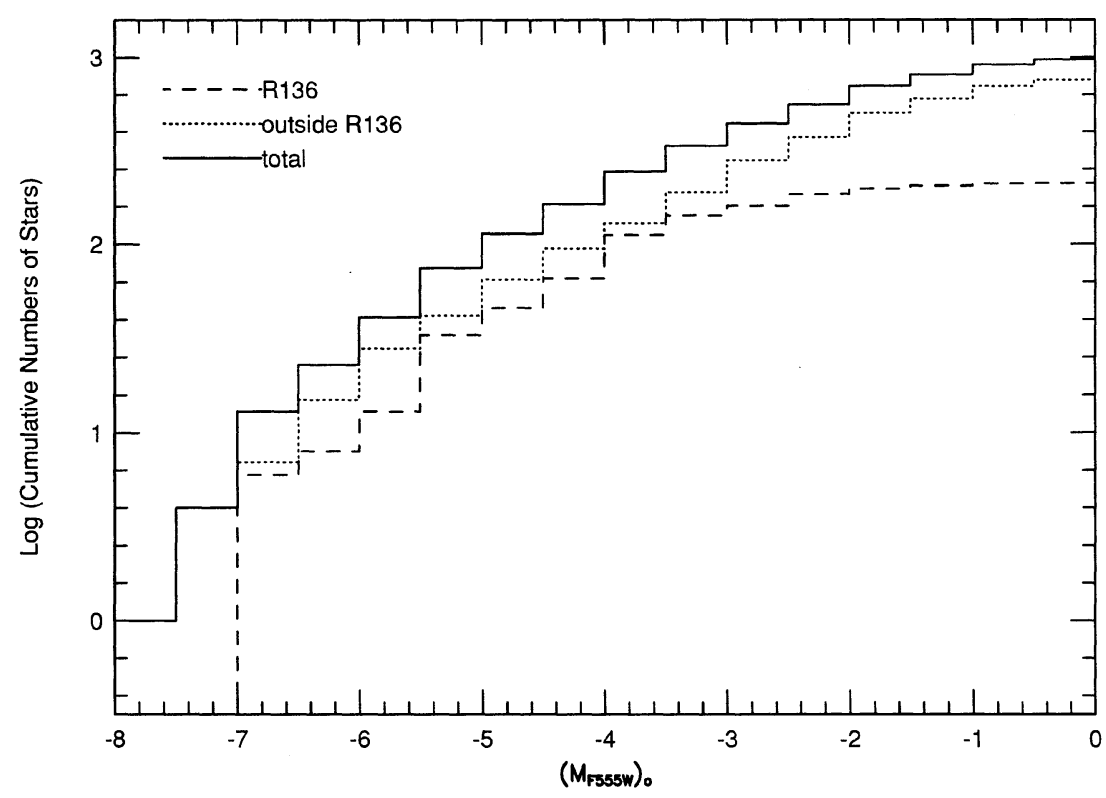

FIG. 12. The $\log$ of the cumulative luminosity function for the absolute, reddening-corrected F555W magnitude. Stars from Tables 2 through 6 have been placed in 0.5 mag bins. 


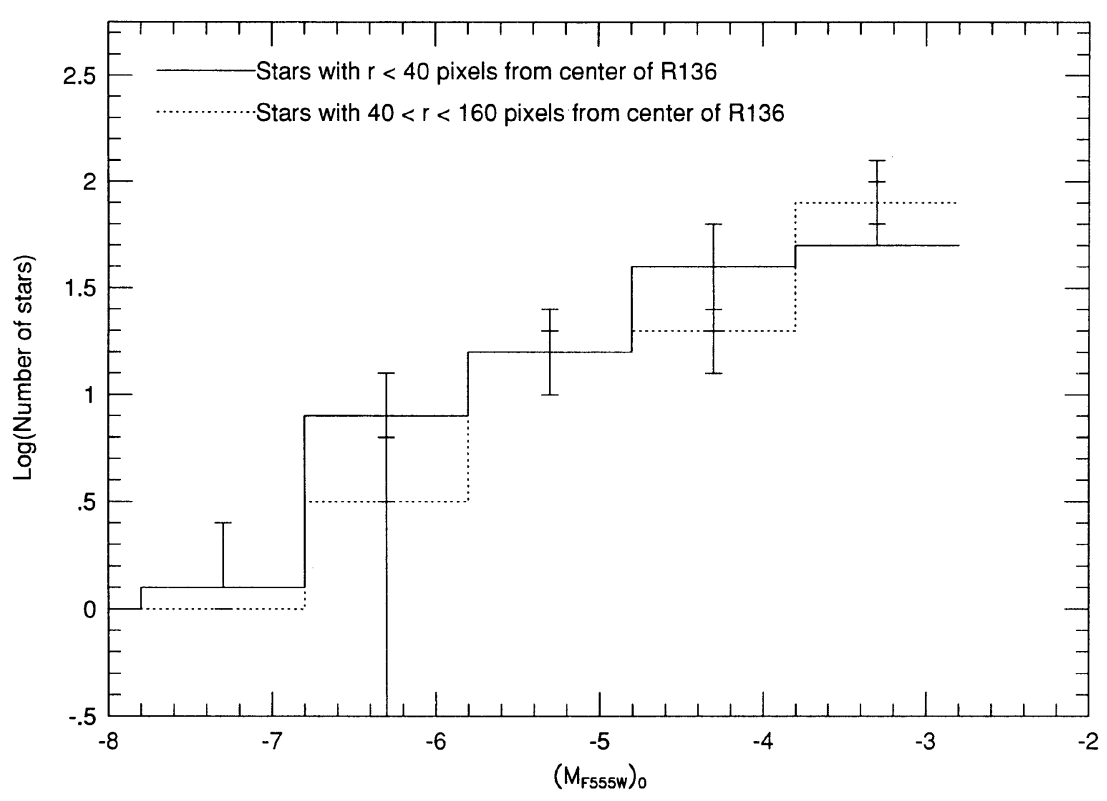

FIG. 13. The F555W luminosity function corrected for incompleteness is shown in one magnitude intervals. The stars are binned into two groups; those within 40 pixels (1.8") of the center of R136 and those in the annulus of 40 to 160 pixels (1.8-7") from the center. The number of stars in the outer region is normalized to that found in the inner region.

where $\sigma$ is the one-dimensional velocity dispersion, $m$ is the typical stellar mass, $\rho$ is the total mass density, and $N$ is the number of stars in the system (Binney \& Tremaine 1987). If we assume $\sigma=r_{c} \sqrt{4 \pi G \rho_{0} / 9}$, then we get

$$
\begin{aligned}
t_{r} \approx & \times 10^{4}\left(\frac{10 \mathscr{M}_{\odot}}{m}\right) \\
& \times\left(\frac{r_{c}}{0.06 \mathrm{pc}}\right)^{3}\left(\frac{\rho_{0}}{5 \times 10^{4} \mathscr{M}_{\odot} \mathrm{pc}^{-3}}\right)^{1 / 2}\left(\frac{1.4}{\ln (0.4 N)}\right) \mathrm{yr} .
\end{aligned}
$$

This time scale is two orders of magnitude shorter than the age of $3.5 \times 10^{6} \mathrm{yrs}$ for the cluster required by the presence of Wolf-Rayet stars, and it will be even shorter for smaller values of $r_{c}$. This suggests that R136a has undergone core collapse and that its present structure is strongly influenced by relaxation effects.

\section{THE CONSEQUENCES OF A LOW MASS STAR POPULATION}

One can estimate the mass in low mass stars that should be present in R136 if the initial mass function is not highly unusual. One can apply a mass-to-light ratio for this system and assume that most of the light arises from the observed stars. This assumption should be quite valid since the observed stars are typically $10^{4}$ times more luminous than the average star. The mass-to-light ratio for a burst model between the ages $3 \times 10^{6}$ and $1 \times 10^{7}$ years is between 0.013 and 0.02 (Charlot \& Bruzual 1991). This corresponds to a total mass in stars between 260 and 400 times the mass in detected stars. With 400 times greater mass

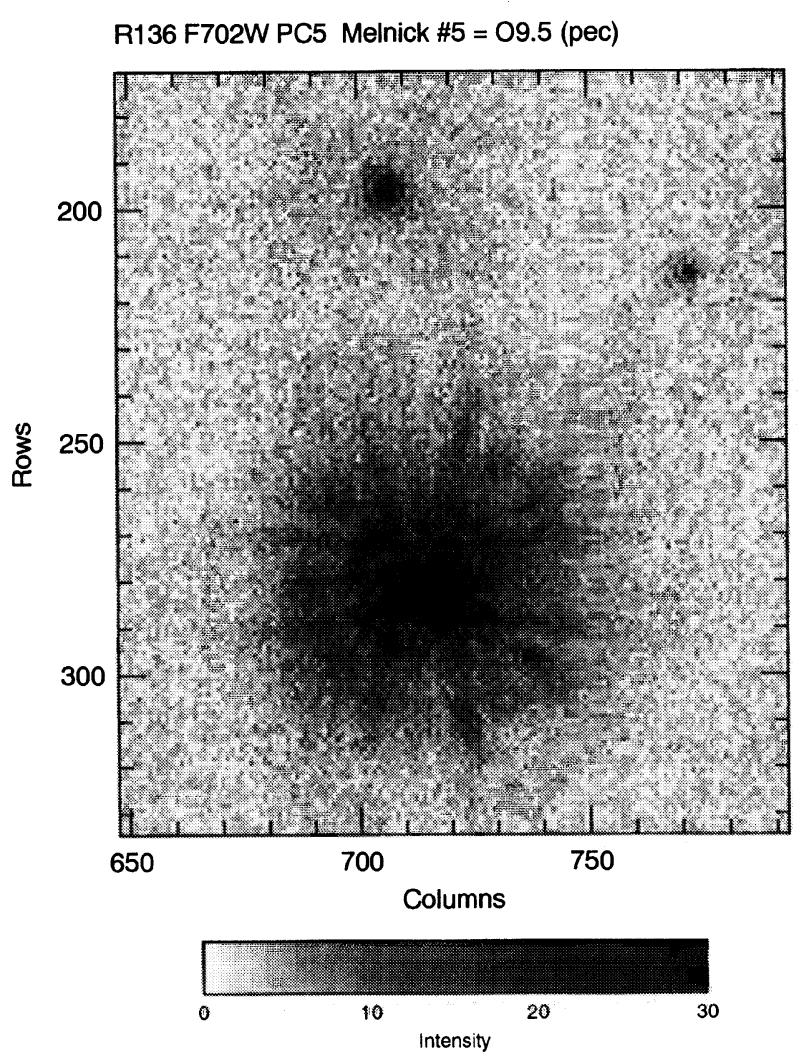

FIG. 14. The image of the peculiar star Mk5 is shown from the F702W frame. The only companion appears to be the much fainter star $1.2^{\prime \prime}$ to the lower right of Mk5. Ten columns are $0.44 "$. 


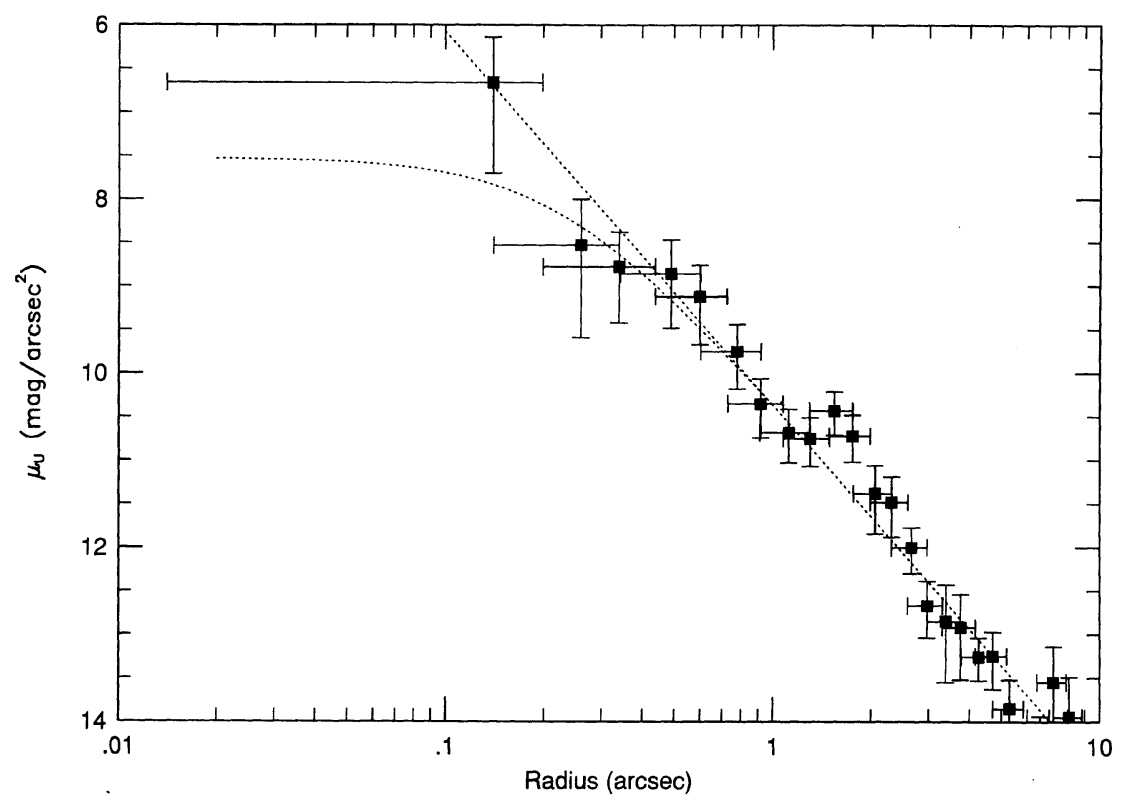

FIG. 15. An F336W surface brightness profile of R136. The upper dotted line shows the form $I(r) \propto r^{\gamma}$ with $\gamma=1.72$; the lower dotted line shows a profile with $r_{c}=0.25^{\prime \prime}$.

and most of that in stars near one solar mass, $t_{\text {relax }}$ grows to $5.0 \times 10^{5}$ years. Thus, a factor of 20 in relaxation time is gained by including the low mass stars. In this case, the R136 core would probably not yet have gone through a core collapse.

Equipartition will occur in a few relaxation times. The result of this is that the high mass stars settle down closer to the center of the cluster than the lighter stars. In equi-

TABLE 9. F336W surface brightness profile.

\begin{tabular}{cccccccr}
\hline \hline $\mathrm{r}\left(^{\prime \prime}\right)$ & $\mathrm{F} 336 \mathrm{~W}^{a}$ & $+\sigma^{b}$ & $-\sigma^{b}$ & $\sigma_{r}^{c}$ & $\sigma_{l}^{c}$ & No. stars & $\mu\left(\mathrm{M}_{\odot} / \mathrm{pc}^{2}\right)^{d}$ \\
\hline 0.14 & 6.66 & -0.52 & 1.04 & -1.000 & 0.151 & 3 & $1.475 \mathrm{E}+04$ \\
0.26 & 8.53 & -0.53 & 1.06 & -0.267 & 0.116 & 3 & $6.097 \mathrm{E}+03$ \\
0.34 & 8.78 & -0.40 & 0.64 & -0.233 & 0.110 & 6 & $7.012 \mathrm{E}+03$ \\
0.49 & 8.86 & -0.39 & 0.62 & -0.156 & 0.090 & 8 & $5.601 \mathrm{E}+03$ \\
0.60 & 9.12 & -0.36 & 0.55 & -0.136 & 0.083 & 10 & $4.810 \mathrm{E}+03$ \\
0.78 & 9.75 & -0.31 & 0.43 & -0.110 & 0.073 & 13 & $3.754 \mathrm{E}+03$ \\
0.92 & 10.35 & -0.29 & 0.39 & -0.100 & 0.068 & 16 & $2.772 \mathrm{E}+03$ \\
1.12 & 10.68 & -0.27 & 0.35 & -0.088 & 0.063 & 19 & $2.200 \mathrm{E}+03$ \\
1.30 & 10.75 & -0.24 & 0.32 & -0.082 & 0.060 & 24 & $2.250 \mathrm{E}+03$ \\
1.54 & 10.43 & -0.22 & 0.28 & -0.075 & 0.056 & 32 & $2.570 \mathrm{E}+03$ \\
1.75 & 10.72 & -0.24 & 0.30 & -0.072 & 0.054 & 28 & $1.876 \mathrm{E}+03$ \\
2.05 & 11.38 & -0.32 & 0.46 & -0.067 & 0.051 & 24 & $1.071 \mathrm{E}+03$ \\
2.30 & 11.48 & -0.29 & 0.40 & -0.065 & 0.050 & 32 & $1.126 \mathrm{E}+03$ \\
2.65 & 12.00 & -0.23 & 0.30 & -0.061 & 0.048 & 42 & $9.554 \mathrm{E}+02$ \\
2.96 & 12.67 & -0.28 & 0.37 & -0.060 & 0.047 & 38 & $5.884 \mathrm{E}+02$ \\
3.38 & 12.85 & -0.42 & 0.70 & -0.057 & 0.045 & 37 & $4.093 \mathrm{E}+02$ \\
3.75 & 12.92 & -0.38 & 0.60 & -0.056 & 0.045 & 44 & $4.017 \mathrm{E}+02$ \\
4.26 & 13.26 & -0.22 & 0.27 & -0.054 & 0.043 & 52 & $3.710 \mathrm{E}+02$ \\
4.70 & 13.25 & -0.28 & 0.38 & -0.053 & 0.043 & 56 & $3.272 \mathrm{E}+02$ \\
5.30 & 13.85 & -0.33 & 0.48 & -0.052 & 0.042 & 51 & $2.184 \mathrm{E}+02$ \\
5.84 & 14.47 & -0.21 & 0.25 & -0.051 & 0.042 & 55 & $1.849 \mathrm{E}+02$ \\
6.56 & 14.16 & -0.22 & 0.28 & -0.050 & 0.041 & 72 & $2.068 \mathrm{E}+02$ \\
7.21 & 13.55 & -0.41 & 0.67 & -0.050 & 0.040 & 70 & $1.720 \mathrm{E}+02$ \\
8.07 & 13.95 & -0.46 & 0.81 & -0.049 & 0.040 & 70 & $1.254 \mathrm{E}+02$ \\
8.85 & 14.90 & -0.23 & 0.29 & -0.049 & 0.040 & 77 & $1.147 \mathrm{E}+02$ \\
9.89 & 14.35 & -0.30 & $\mathbf{0 . 4 2}$ & -0.048 & 0.039 & 76 & $1.077 \mathrm{E}+02$ \\
\hline
\end{tabular}

NOTE.-

${ }^{a}$ F $336 \mathrm{~W}$ magnitudes corrected by $\mathrm{A}_{336}$.

${ }^{b}$ Uncertainties in the F336W magnitudes.

cLeft and right radial bin error ( $\log$ units). Each point is at the center of a radial bin. The left error extends to the inner radius of the bin, the right to the outer.

${ }^{d}$ Surface mass density. librium, the $10 \mathscr{M}_{\odot}$ stars will sink to a mean radius of one third the cluster radius defined by the low mass stars. The relevant core radius is therefore somewhat larger than 0.06 pc and subsequently the relaxation time is somewhat longer still. This effect, plus the fact that there have been several full relaxation periods, are indications that the core densities have risen substantially from their initial values and help to explain how such high present densities of high mass stars were obtained. The original core density was probably at least 30 times lower than the present one.

Since the high end slope of the initial mass function is different in R136 and the densities are very high, a natural question to ask is, could collisional mergers have altered the initial mass function? With $1.6 \times 10^{5}$ stars in a $0.25 \mathrm{pc}$ radius, the collision time per star is $10^{11}$ years. If we say the system is $10^{7}$ years old, then one in $10^{4}$ stars has undergone a collision. This implies that there have been 14 collisions. Therefore, collisional merger is unlikely to have substantially altered the initial mass function.

\section{SUMMARY}

We have used images obtained with the Planetary Camera on the $H S T$ through filters F368M, F336W, F555W, F702W, and F469N to explore the stellar population of $\mathrm{R} 136$, the core of the 30 Doradus cluster. We found that $\mathrm{R} 136 \mathrm{a}$, the brightest knot at the center of R136, is indeed a tight cluster of stars, containing at least 12 components in a 1 " region. Three of these stars are of the Wolf-Rayet type. None of the stars in R136a is individually remarkable in terms of its luminosity or color compared to blue massive stars observed elsewhere in 30 Doradus and in other 
galaxies. The brightest stars have luminosities consistent with their being massive $O$ supergiants or Wolf-Rayet stars. In R136a the stellar mass density is at least a million times that of the solar neighborhood and three times that in the core of NGC 3603 . The luminosity density is at least 50 times that of the core $(0.2 \mathrm{pc})$ of Orion and seven times that in NGC 3603's inner 0.34 pc.

In the larger region known as R136 we detected and measured the magnitudes of 214 stars. A color-magnitude diagram in the F336W and F555W filters shows a range in stars from luminous O supergiants to ZAMS B3 stars. The color-magnitude diagram of the stars within $R 136$ is very similar to that of stars outside of R136. There is a spread in $\left(M_{336}-M_{555}\right)_{0}$ of about $0.5 \mathrm{mag}$ which is probably partly intrinsic. A clump of redder stars at the faint end is probably due to early ZAMS B stars, and a turn to the red at the bright end could mark the main-sequence turnoff. No red supergiants were detected within R136. An F555W luminosity function also shows the similarity between the types of stars within and outside R136 except for $M_{555}$ $>-4$, at which point the luminosity function becomes incomplete in $\mathrm{R} 136$ since fainter stars are harder to detect there. The slope of the upper end of the luminosity function is flatter than that found in Constellation III or in galaxy-wide surveys, due at least in part to the very young age of 30 Doradus. The total luminosity per unit area in $\mathbf{R} 136$ is found to be at least comparable to that in the core of Orion, but in $\mathrm{R} 136$ this density is sustained over a region that is 130 times larger.

An F336W surface brightness profile constructed from the stellar photometry was found to be best fit by a pure power law with $I(r) \propto r^{\gamma}$ with $\gamma=-1.72 \pm 0.06$. However, because of the small numbers of stars at the very center, the brightness profile is also consistent with a very small core with $r_{c}<0.25^{\prime \prime}$, considerably smaller than that estimated by Moffat et al. (1985) from ground-based observations. If $r_{c}=0.25^{\prime \prime}, \rho_{0}>5 \times 10^{4} \mathscr{M}_{\odot} \mathrm{pc}^{-3}$ including only stars with $M_{555}>-0.5$ and incomplete for $M_{555}>-4$. The upper limit on the relaxation time for the cluster implied by this $r_{c}$ and central density is 100 times shorter than the age required by the presence of Wolf-Rayet stars, suggesting that relaxation effects have been very important in the observed structure of the cluster unless a large population of lower mass stars is present.

Although we have confirmed that the individual stars in R136a and in R136 are themselves unremarkable, we must stress that they are part of a most remarkable star-forming region. What is extreme is not the masses of the individual stars, but that there is such a high concentration of the most massive stars made anywhere. There are something like 160 stars in R136 that are more massive than about $10 \mathscr{M}_{\odot}$, at least 8 of which are more massive than $40 \mathscr{M}_{\odot}$. And, all of this is within 2 pc. Outside of R136 there are also large numbers of very massive stars: $77 \mathrm{O}$ stars, including five of the O3If-type, 11 Wolf-Rayet stars, and a smattering of red and blue supergiants (Walborn 1991). This raises the question: How does the natal cloud contract to a density necessary to form R136 without breaking up into stars sooner? Furthermore, what will be the consequence to the Large Magellanic Cloud?

The latter question is a little easier to address. Since massive stars live such short lifetimes, we expect that the stars within R136 formed pretty much all at the same time. That means that there will be several hundred supernovae exploding over a period of perhaps ten million years. The energy released is likely to have a major impact on the surrounding interstellar medium. It has been argued that 30 Doradus is on its way to blowing a hole in the interstellar medium (cf. Cohen et al. 1988), such as the stars in Constellation III have done (Dopita et al. 1985). 30 Doradus will probably also become a galactic fountain in the end, like LMC2, the supergiant shell to the east of 30 Doradus (Wang \& Helfand 1991). Certainly, a starforming event such as 30 Doradus is a major perturbation in a small galaxy like the LMC.

B.C. gratefully acknowledges the unfailing logistical and moral support of E. Jaderlund and S. Durham from her home institution. We are also grateful to $\mathrm{T}$. Kreidl who made the original suggestion to include R136 among the GTO targets. This work was conducted by the WF/PC Investigation Definition Team, under NASA Contract No. NAS5-25421.

\section{REFERENCES}

Baier, G., Ladebeck, R., \& Weigelt, G. 1985, A\&A, 151, 61

Binney, J., \& Tremaine, S. 1987, Galactic Dynamics (Princeton University Press, Princeton)

Charlot, S., \& Bruzual A., G. 1991, ApJ, 367, 126

Cassinelli, J. P., Mathis, J. S., \& Savage, B. D. 1981, Science, 212, 1497

Chu, Y.-H, Cassinelli, J. P., \& Wolfire, M. G. 1984, ApJ, 283, 560

Cohen, R. S., Dame, T. M., Garay, G., Montani, J., Rubio, M., \& Thaddeus, P. 1988, ApJ, 331, L95

Conti, P. S., \& Underhill, A. B. (eds.) 1988, O Stars and Wolf-Rayet Stars, NASA SP-497 (NASA Monograph Series, NASA)

Dopita, M. A., Mathewson, D. S., \& Ford, V. L. 1985, ApJ, 297, 599

Edvardsson, B., \& Bell, R. A. 1989, MNRAS, 238, 1121

Feitzinger, J. V., Schlosser, W., Schmidt-Kaler, Th., \& Winkler, C. 1980, A\&A, 84, 50
Fitzpatrick, E. L., \& Savage, B. D. 1984, ApJ, 279, 578

Flower, P. J. 1977, A\&A, 54, 31

Freedman, W. L. 1985, ApJ, 299, 74

Griffiths, R. 1989, HST Wide Field and Planetary Camera Instrument Handbook (Space Telescope Science Institute, Baltimore)

Harris, H. C., Baum, W. A., Hunter, D. A., \& Kreidl, T. J. 1991, AJ, 101, 677

Johnson, H. 1968, ARA\&A, 4, 196

Kennicutt, R. C. 1991, in The Magellanic Clouds, edited by R. Haynes and D. Milne (Kluwer, Netherlands), p. 139

Lucy, L. B. 1974, AJ, 79, 745

Maeder, A., \& Meynet, G. 1987, A\&A, 182, 243

Massey, P. 1985, PASP, 97, 5

Melnick, J. 1985, A\&A, 153, 235 
Melnick, J. 1986, in Star-forming Dwarf Galaxies and Related Objects, edited by D. Kunth, T. X. Thuan, and J. T. Thanh Van (Éditions Frontières, Paris), p. 171

Moffat, A. J. 1983, A\&A, 124, 273

Moffat, A. J., \& Seggewiss, W. 1983, A\&A, 125, 83

Moffat, A. J., Seggewiss, W., \& Shara, M. M. 1985, ApJ, 295, 109

Morton, D. C., \& Adams, T. F. 1968, ApJ, 151, 611

Neri, R., \& Grewing, M. 1988, A\&A, 196, 338

Parker, J. 1992, Ph.D thesis, University of Colorado

Ratnatunga, K. U., \& Bahcall, J. N. 1985, ApJS, 59, 63

Reid, N., Mould, J., \& Thompson, I. 1987, ApJ, 323, 433

Savage, B. D., Fitzpatrick, E. L., Cassinelli, J. P., \& Ebbets, D. C. 1983, ApJ, 273, 597

Scalo, J. S. 1986, Fund Cosmic Phys., 11, 1

Schaller, G., Schaerer, D., Meynet, G., \& Maeder, A. 1992, preprint
Stetson, P. B. 1987, PASP, 99, 191

Stothers, R. B., \& Chin, C.-W. 1977, ApJ, 211, 189

Thronson, H. A., et al. 1986, AJ, 91, 1350

Walborn, N. R. 1973, ApJ, 182, L21

Walborn, N. R. 1986, in Luminous Stars and Associates in Galaxies, edited by C. W. H. de Loore, A. J. Willis, and P. Laskarides (Reidel, Dordrecht), p. 185

Walborn, N. R. 1991, in The Magellanic Clouds, edited by R. Haynes and D. Milne (Kluwer, Netherlands), p. 145

Wang, Q., \& Helfand, D. J. 1991, ApJ, 370, 541

Weigelt, G., et al. 1991, ApJ, 378, L21

Weigelt, G., \& Baier, G. 1985, A\&A, 150, L18

WF/PC Investigation Definition Team 1992, Final Orbital/Science Verification Report, a report to Space Telescope Science Institute 

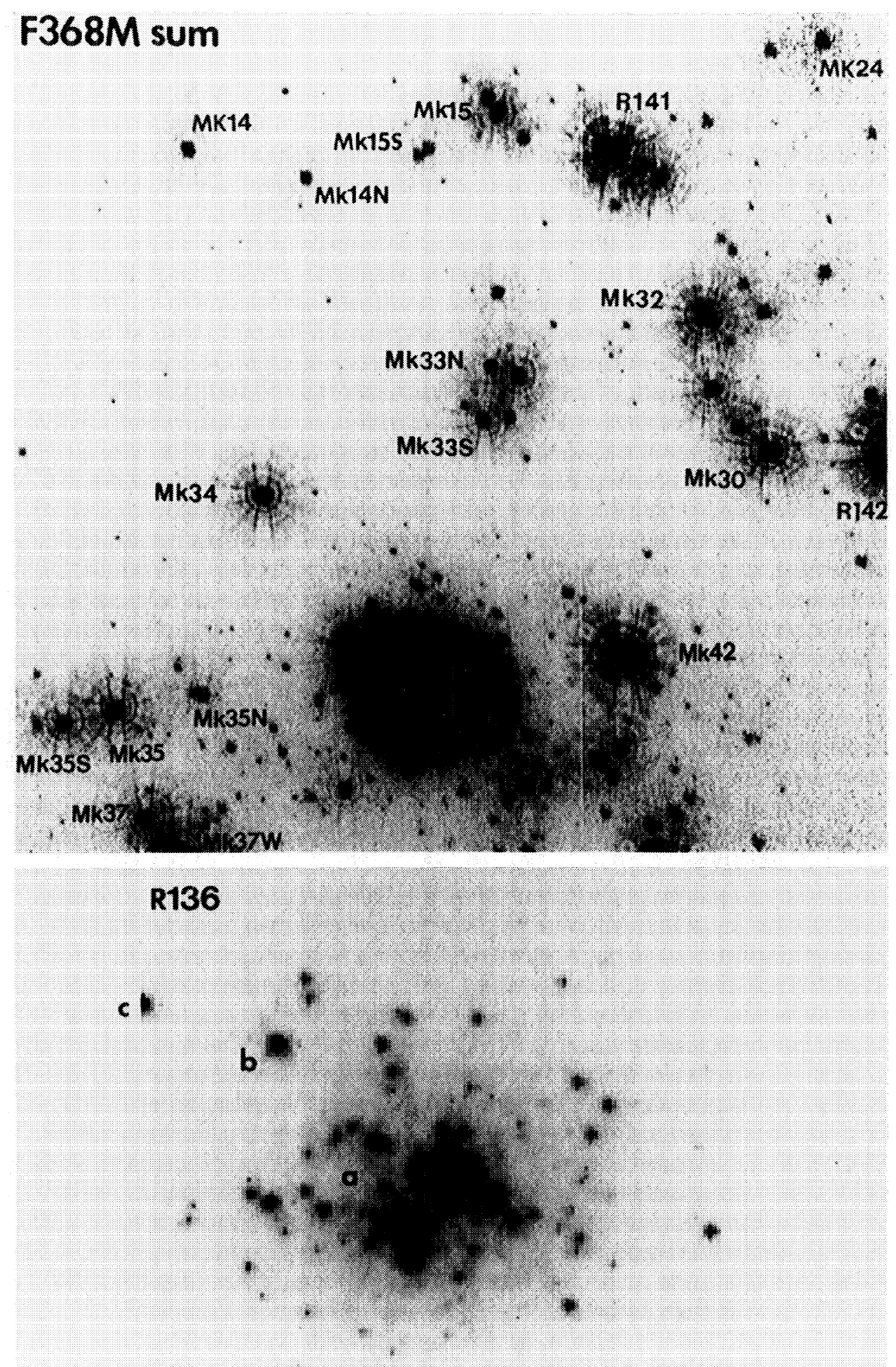

FIG. 1. The top frame is the full $800 \times 800$ (35") PC6 F368M image of the 30 Doradus field containing $\mathrm{R} 136$. The field is oriented such that north is rotated about $65^{\circ}$ counterclockwise. Stars catalogued by Melnick (1985) are identified as "Mk." The bottom panel shows the inner 5.6" of the cluster from the same image. R136a, b, and c (Feitzinger et al. 1980) are identified. 
6.

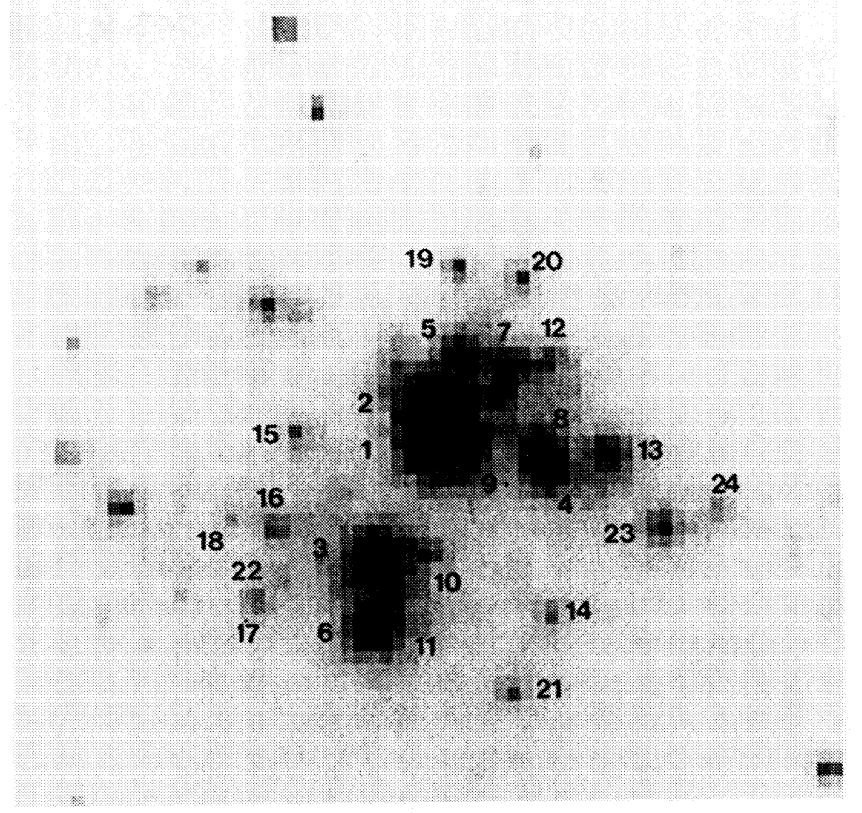

\section{Deconvolved}

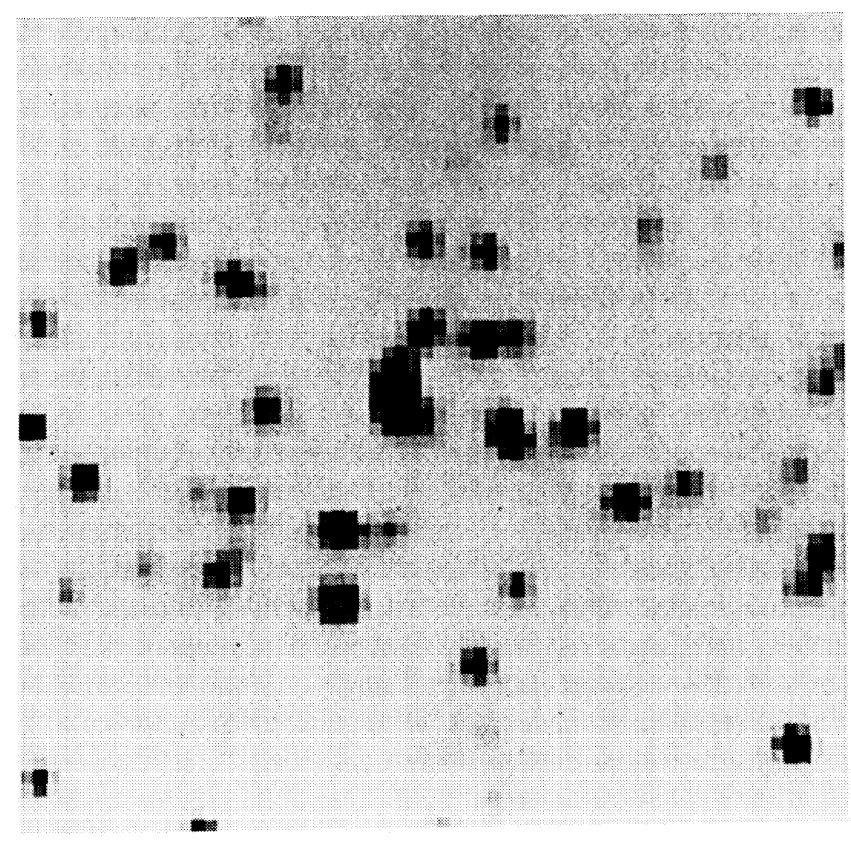

FIG. 2. (a) The top frame displays a $2.8^{\prime \prime}$ portion of the F368M image so that the individual stars in R136a can be seen. The stars in approximately the inner $1.5^{\prime \prime}$ are numbered starting with eight components identified by Weigelt \& Baier (1985) from speckle observations. (b) The bottom frame shows the same field after deconvolution using the Lucy (1974) algorithm. 


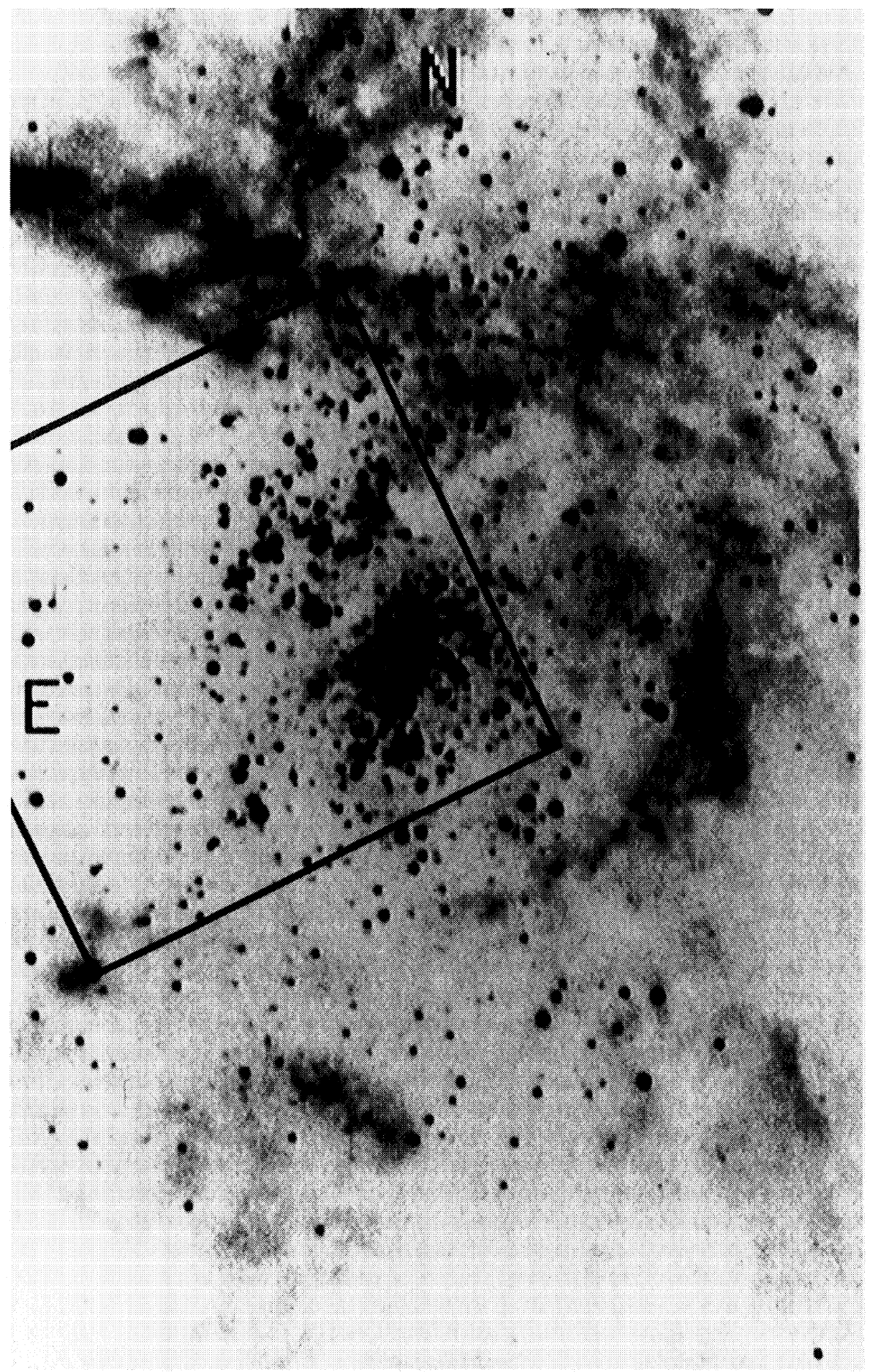

FIG. 3. The field of view and orientation of the PC (all four CCD's) in the 1991 images is outlined on a ground-based $V$-band image of the region which was kindly loaned to the WF/PC team. The field is 67 " on a side. 

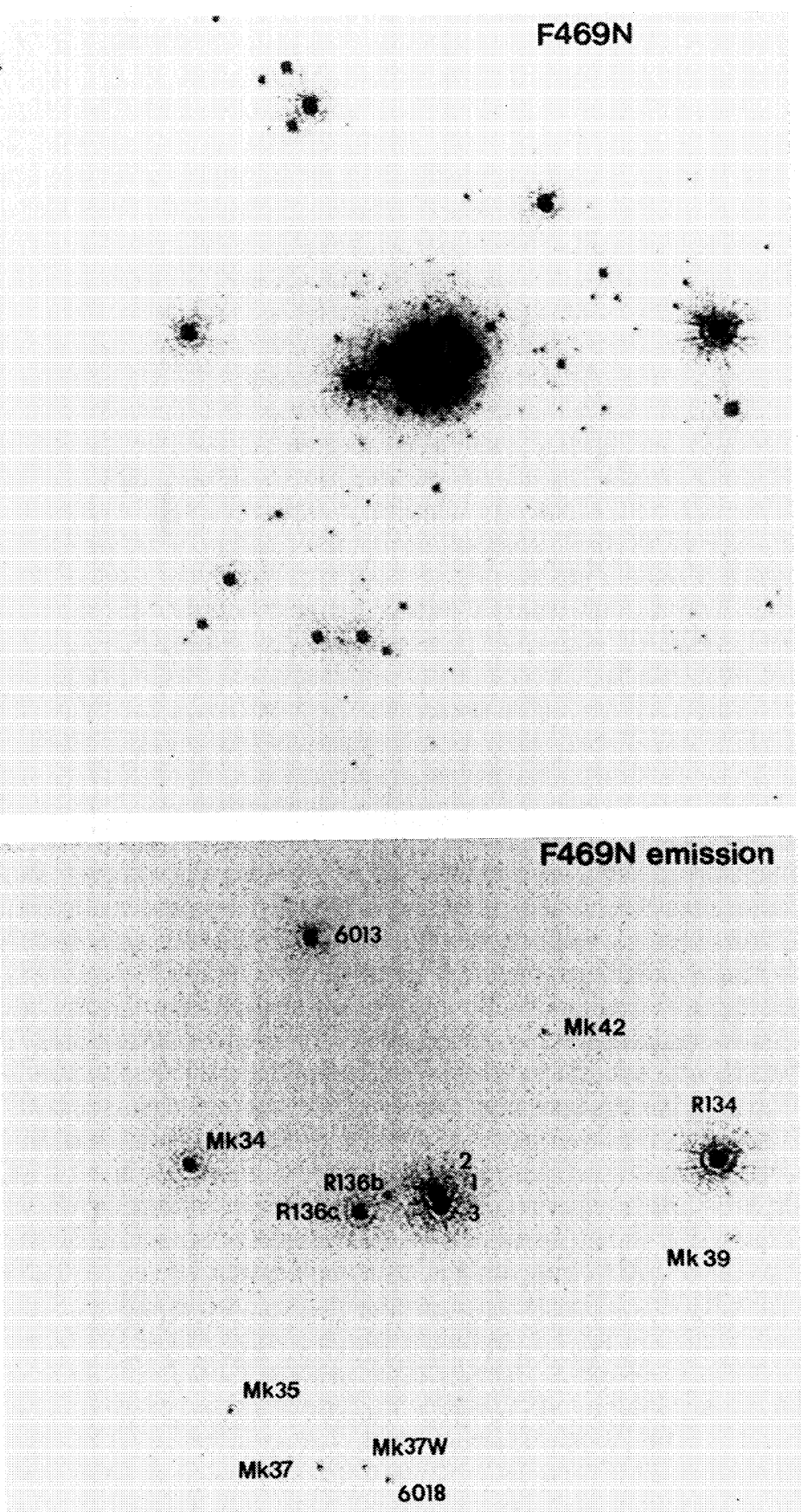

FIG. 6. The F469N PC6 images are shown. The top panel is the unsubtracted image; the bottom image has had the stellar continuum subtracted from it to leave only He II $\lambda 4686$ emission. 